\title{
CONSTRUCTION OF A MEAN SQUARE ERROR ADAPTIVE EULER-MARUYAMA METHOD WITH APPLICATIONS IN MULTILEVEL MONTE CARLO
}

\author{
HÅKON HOEL ${ }^{\dagger, \ddagger}$, JUHO HÄPPÖLÄ ${ }^{\dagger}$, AND RAÚL TEMPONE ${ }^{\dagger}$
}

\begin{abstract}
A formal mean square error expansion (MSE) is derived for Euler-Maruyama numerical solutions of stochastic differential equations (SDE). The error expansion is used to construct a pathwise, a posteriori, adaptive time-stepping Euler-Maruyama algorithm for numerical solutions of SDE, and the resulting algorithm is incorporated into a multilevel Monte Carlo (MLMC) algorithm for weak approximations of SDE. This gives an efficient MSE adaptive MLMC algorithm for handling a number of low-regularity approximation problems. In low-regularity numerical example problems, the developed adaptive MLMC algorithm is shown to outperform the uniform time-stepping MLMC algorithm by orders of magnitude, producing output whose error with high probability is bounded by TOL $>0$ at the near-optimal MLMC cost rate $\mathcal{O}\left(\mathrm{TOL}^{-2} \log (\mathrm{TOL})^{4}\right)$.
\end{abstract}

\section{Contents}

1. Introduction

1.1. Monte Carlo Methods and the Euler-Maruyama Scheme.

1.2. Uniform and Adaptive Time-Stepping MLMC

2. Derivation of the MSE A Posteriori Adaptive Algorithm

2.1. The Error Expansion

2.2. The Adaptive Algorithm 10

2.3. Numerical Examples 13

3. Extension of the Adaptive Algorithm to the Multilevel Setting

3.1. Notation and Objective 16

3.2. Error Control 17

3.3. MLMC Pseudocode 19

4. Numerical Examples for the MLMC Algorithms 20

5. Conclusion 25

Appendix A. Theoretical Results 22

A.1. Error Expansion for the MSE in 1D 22

A.2. Second, Third and Fourth Variations 36

A.3. Error Expansion for the MSE in Multiple Dimensions

Appendix B. A Uniform Time Step MLMC Algorithm

References 42

2010 Mathematics Subject Classification. Primary 65C20; Secondary 65 C05.

Key words and phrases. Adaptive time stepping, stochastic differential equations, multilevel Monte Carlo.

$\dagger$ Computer, Electrical and Mathematical Sciences and Engineering (CEMSE) Division King Abdullah University of Science and Technology, Thuwal 23955-6900, Kingdom of Saudi Arabia (juho.happloa@kaust.edu.sa, raul.tempone@kaust.edu.sa).

$\ddagger$ Department of Mathematics, University of Oslo, P.O. Box1053, Blindern, NO-0316 Oslo, Norway (Corresponding author: haakonah@math.uio.no). 


\section{INTRODUCTION}

SDE models are frequently applied in mathematical finance [29, 28, 12], where an observable may, for example, represent the payoff of an option. SDE are also used to model the dynamics of multiscale physical, chemical or biochemical systems [11, 25, 30, 32, where, for instance, concentrations, temperature and energy may be sought observables.

Given a filtered, complete probability space $\left(\Omega, \mathcal{F},\left(\mathcal{F}_{t}\right)_{0 \leqslant t \leqslant T}, \mathrm{P}\right)$, we consider the Itô SDE

$$
\begin{aligned}
d X_{t} & =a\left(t, X_{t}\right) d t+b\left(t, X_{t}\right) d W_{t}, \quad t \in(0, T], \\
X_{0} & =x_{0},
\end{aligned}
$$

where $X:[0, T] \times \Omega \rightarrow \mathbb{R}^{d_{1}}$ is a stochastic process with randomness generated by a $K$-dimensional Wiener process, $W:[0, T] \times \Omega \rightarrow \mathbb{R}^{d_{2}}$, with independent components, $W=\left(W^{(1)}, W^{(2)}, \ldots, W^{\left(d_{2}\right)}\right)$, and $a:[0, T] \times \mathbb{R}^{d_{1}} \rightarrow \mathbb{R}^{d_{1}}$ and $b:[0, T] \times \mathbb{R}^{d_{1}} \rightarrow \mathbb{R}^{d_{1} \times d_{2}}$ are the drift and diffusion coefficients, respectively. The considered filtration $\mathcal{F}_{t}$ is generated from the history of the Wiener process $W$ up to time $t$ and the possible outcomes of the initial data $X_{0}$, and succeedingly completed with all P-outer measure zero sets of the sample space $\Omega$. That is

$$
\mathcal{F}_{t}:=\overline{\sigma\left(\left\{W_{s}\right\}_{0 \leqslant s \leqslant t}\right) \vee \sigma\left(X_{0}\right)}
$$

where the operation $\mathcal{A} \vee \mathcal{B}$ denotes the $\sigma$-algebra generated by the pair of $\sigma$-algebras $\mathcal{A}$ and $\mathcal{B}$, i.e., $\mathcal{A} \vee \mathcal{B}:=\sigma(\mathcal{A}, \mathcal{B})$, and $\overline{\mathcal{A}}$ denotes the P-outer measure null-set completion of $\mathcal{A}$,

$$
\overline{\mathcal{A}}:=\mathcal{A} \vee\left\{A \subset \Omega \mid \inf _{\hat{A} \in\{\check{A} \in \mathcal{A} \mid \check{A} \supset A\}} \mathrm{P}(\hat{A})=0\right\} .
$$

The contributions of this work are twofold. First, an a posteriori adaptive time-stepping algorithm for computing numerical realizations of SDE using the Euler-Maruyama method is developed. And second, for a given observable $g: \mathbb{R}^{d_{1}} \rightarrow \mathbb{R}$, we construct a mean square error (MSE) adaptive time-stepping multilevel Monte Carlo (MLMC) algorithm for approximating the expected value, $\mathrm{E}\left[g\left(X_{T}\right)\right]$, under the following constraint:

$$
\mathrm{P}\left(\left|\mathrm{E}\left[g\left(X_{T}\right)\right]-\mathcal{A}\right| \leqslant \mathrm{TOL}\right) \geqslant 1-\delta .
$$

Here, $\mathcal{A}$ denotes the algorithm's approximation of $\mathrm{E}\left[g\left(X_{T}\right)\right]$ (examples of which are given in Equations (1.1) and (6)) and TOL and $\delta>0$ are accuracy and confidence constraints, respectively.

The rest of this paper is organized as follows: First, in Section 1.1, we review the Monte Carlo methods and their use with the Euler-Maruyama integrator. This is followed by discussion of Multilevel Monte Carlo methods and adaptivity for SDEs. The theory, framework and numerical examples for the MSE adaptive algorithm is presented in Section 2, In Section 3, we develop the framework for the MSE adaptive MLMC algorithm and present implementational details in algorithms with pseudocode. In Section 4, we compare the performance of the MSE adaptive and uniform MLMC algorithms in a couple of numerical examples, one of which is a low-regularity SDE problem. Finally, we present brief conclusions followed by technical proofs and the extension of the main result to higher-dimensional problems in the appendices.

1.1. Monte Carlo Methods and the Euler-Maruyama Scheme. Monte Carlo (MC) methods provide a robust and typically non-intrusive way to compute weak approximations of SDE. The convergence rate of MC methods does not depend on the dimension of the problem; for that reason, $\mathrm{MC}$ is particularly effective on multi-dimensional problems. In its simplest form, an approximation by the MC method consists of the following two steps:

(A.1) Compute $M$ independent and identically distributed numerical realizations, $\bar{X}_{T}\left(\omega_{m}\right)$, of the SDE (1).

(A.2) Approximate $\mathrm{E}\left[g\left(X_{T}\right)\right]$ by the sample average

$$
\mathcal{A}:=\sum_{m=1}^{M} \frac{g\left(\bar{X}_{T}\left(\omega_{m}\right)\right)}{M} .
$$


Regarding ordinary differential equations (ODE), the theory for numerical integrators of different orders for scalar SDE is vast. Provided sufficient regularity, higher order integrators generally yield higher convergence rates [22]. With MC methods it is straightforward to determine that the goal 22 is fulfilled at the computational cost $\mathcal{O}\left(\mathrm{TOL}^{-2-1 / \alpha}\right)$, where $\alpha \geqslant 0$ denotes the weak convergence rate of the numerical method, as defined in Equation (4).

As a method of temporal discretization, the Euler-Maruyama scheme is given by

$$
\begin{aligned}
\bar{X}_{t_{n+1}} & =\bar{X}_{t_{n}}+a\left(t_{n}, \bar{X}_{t_{n}}\right) \Delta t_{n}+b\left(t_{n}, \bar{X}_{t_{n}}\right) \Delta W_{n}, \\
\bar{X}_{0} & =x_{0},
\end{aligned}
$$

using time steps $\Delta t_{n}=t_{n+1}-t_{n}$ and Wiener increments $\Delta W_{n}=W_{t_{n+1}}-W_{t_{n}} \sim N\left(0, \Delta t_{n} I_{d_{2}}\right)$, where $I_{d_{2}}$ denotes the $d_{2} \times d_{2}$ identity matrix. In this work, we will focus exclusively on EulerMaruyama time-stepping. The Euler-Maruyama scheme, which may be considered the SDEequivalent of the forward-Euler method for ODE, has, under sufficient regularity, first-order weak convergence rate

$$
\left|\mathrm{E}\left[g\left(X_{T}\right)-g\left(\bar{X}_{T}\right)\right]\right|=\mathcal{O}\left(\max _{n} \Delta t_{n}\right),
$$

and also first-order MSE convergence rate

$$
\mathrm{E}\left[\left(g\left(X_{T}\right)-g\left(\bar{X}_{T}\right)\right)^{2}\right]=\mathcal{O}\left(\max _{n} \Delta t_{n}\right),
$$

cf. 22. For multi-dimensional SDE problems, higher order schemes are generally less applicable, as either the diffusion coefficient matrix has to fulfill a rigid commutativity condition, or Levy areas, required in higher order numerical schemes, have to be accurately approximated to achieve better convergence rates than those obtained with the Euler-Maruyama method 22 .

1.2. Uniform and Adaptive Time-Stepping MLMC. MLMC is a class of MC methods that uses a hierarchy of subtly correlated and increasingly refined realization ensembles to reduce the variance of the sample estimator. In comparison with single-level MC, MLMC may yield orders of magnitude reductions in the computational cost of moment approximations. MLMC was first introduced by Heinrich [14, 15] for approximating integrals that depend on random parameters. For applications in SDE problems, Kebaier 21] introduced a two-level MC method and demonstrated its potential efficiency gains over single-level MC. Giles [8] thereafter developed an MLMC algorithm for SDE, exhibiting even higher potential efficiency gains. Presently, MLMC is a vibrant and growing research topic, (cf. 10, 9, 3, 4, 34, 26, 13], and references therein).

1.2.1. MLMC Notation. We define the multilevel estimator by

$$
\mathcal{A}_{\mathcal{M L}}:=\sum_{\ell=0}^{L} \sum_{i=1}^{M_{\ell}} \frac{\Delta_{\ell} g\left(\omega_{i, \ell}\right)}{M_{\ell}},
$$

where

$$
\Delta_{\ell} g(\omega):= \begin{cases}g\left(\bar{X}_{T}^{\{0\}}(\omega)\right), & \text { if } \ell=0, \\ g\left(\bar{X}_{T}^{\{\ell\}}(\omega)\right)-g\left(\bar{X}_{T}^{\{\ell-1\}}(\omega)\right), & \text { otherwise. }\end{cases}
$$

Here, the positive integer, $L$, denotes the final level of the estimator, $M_{\ell}$ is the number of sample realizations on the $\ell$-th level, and the realization pair, $\bar{X}_{T}^{\{\ell\}}\left(\omega_{i, \ell}\right)$ and $\bar{X}_{T}^{\{\ell-1\}}\left(\omega_{i, \ell}\right)$, are generated by the Euler-Maruyama method (3) using the same Wiener path, $W\left(\omega_{i, \ell}\right)$, sampled on the respective meshes, $\Delta t^{\{\ell\}}$ and $\Delta t^{\{\ell-1\}}$, (cf. Figure 1). For consistency, we also introduce the notation $W^{\{\ell\}}(\omega)$ for the Wiener path restricted to the mesh $\Delta t^{\{\ell\}}(\omega)$.

1.2.2. Uniform Time-Stepping MLMC. In the uniform time-stepping MLMC introduced in [8], the respective SDE realizations $\left\{\bar{X}_{T}^{\{\ell\}}\right\}_{\ell}$ are constructed on a hierarchy of uniform meshes with geometrically decaying step size, $\min \Delta t^{\{\ell\}}=\max \Delta t^{\{\ell\}}=T / N_{\ell}$, and $N_{\ell}=c^{\ell} N_{0}$ with $c \in \mathbb{N} \backslash\{1\}$ and $N_{0}$ a finite integer. For simplicity, we consider the uniform time-stepping MLMC method with $c=2$. 

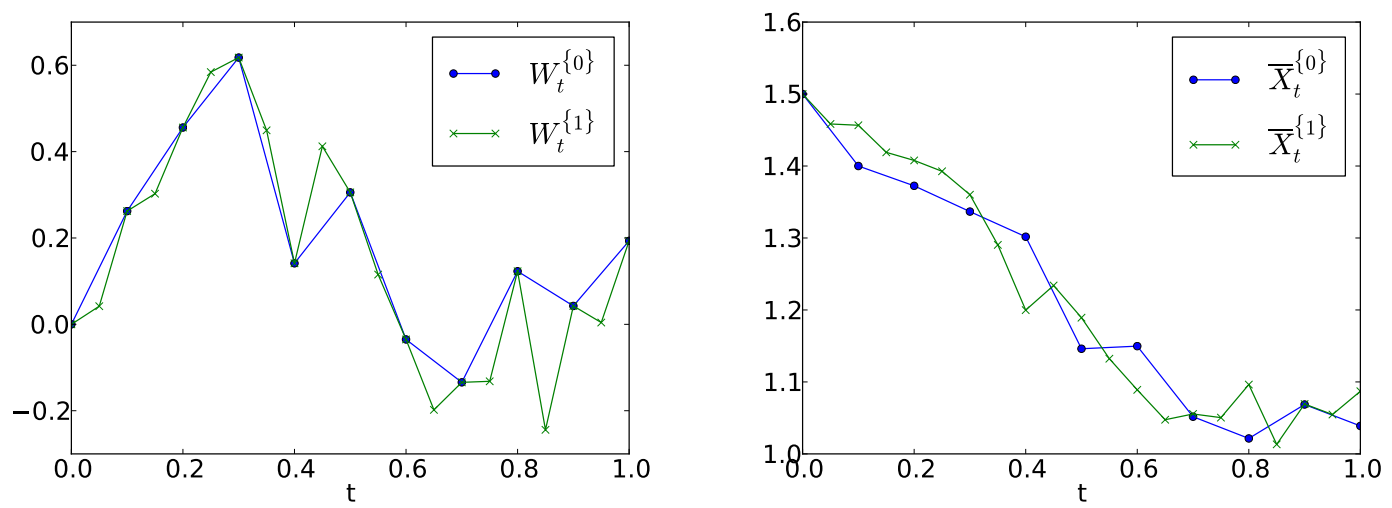

Figure 1. (Left) A sample Wiener path, $W$, generated on the coarse mesh, $\Delta t^{\{0\}}$, with uniform step size $1 / 10$ (blue line ). The path is thereafter Brownian bridge interpolated onto a finer mesh, $\Delta t^{\{1\}}$, which has uniform step size of 1/20 (green line). (Right) Euler-Maruyama numerical solutions of the OrnsteinUhlenbeck SDE problem, $d X_{t}=2\left(1-X_{t}\right) d t+0.2 d W_{t}$, with initial condition $X_{0}=3 / 2$, are computed on the meshes $\Delta t^{\{0\}}$ (blue line) and $\Delta t^{\{1\}}$ (green line) using Wiener increments from the respective path resolutions.

1.2.3. Uniform Time-Stepping MLMC Error and Computational Complexity. By construction, the multilevel estimator is telescoping in expectation, i.e., $\mathrm{E}\left[\mathcal{A}_{\mathcal{M L}}\right]=\mathrm{E}\left[g\left(\bar{X}_{T}^{\{L\}}\right)\right]$. Using this property, we may conveniently bound the multilevel approximation error:

$$
\left|\mathrm{E}\left[g\left(X_{T}\right)\right]-\mathcal{A}_{\mathcal{M L}}\right| \leqslant \underbrace{\left|\mathrm{E}\left[g\left(X_{T}\right)-g\left(\bar{X}_{T}^{\{L\}}\right)\right]\right|}_{=: \mathcal{E}_{T}}+\underbrace{\left|\mathrm{E}\left[g\left(\bar{X}_{T}^{\{L\}}\right)-\mathcal{A}_{\mathcal{M C}}\right]\right|}_{=: \mathcal{E}_{S}} .
$$

The approximation goal (2) is then reached by ensuring that the sum of the bias, $\mathcal{E}_{T}$, and the statistical error, $\mathcal{E}_{S}$, is bounded from above by TOL, e.g., by the constraints $\mathcal{E}_{T} \leqslant$ TOL $/ 2$ and $\mathcal{E}_{S} \leqslant \mathrm{TOL} / 2$, (see Section 3.2 for more details on the MLMC error control). For the MSE error goal,

$$
\mathrm{E}\left[\left(\mathrm{E}\left[g\left(X_{T}\right)\right]-\mathcal{A}_{\mathcal{M L}}\right)^{2}\right] \leqslant \mathrm{TOL}^{2}
$$

the following theorem states the optimal computational cost for MLMC:

Theorem 1.1 (Computational cost of deterministic MLMC; Cliffe et al. [4]). Suppose there are constants $\alpha, \beta, \gamma$ such that $\alpha \geqslant \frac{\min (\beta, \gamma)}{2}$ and

(ii) $\operatorname{Var}\left(\Delta_{\ell} g\right)=\mathcal{O}\left(N_{\ell}^{-\beta}\right)$,

(iii) $\operatorname{Cost}\left(\Delta_{\ell} g\right)=\mathcal{O}\left(N_{\ell}^{\gamma}\right)$.

Then, for any TOL $<e^{-1}$, there exists an $L$ and a sequence $\left\{M_{\ell}\right\}_{\ell=0}^{L}$ such that

$$
\mathrm{E}\left[\left(\mathcal{A}_{\mathcal{M L}}-\mathrm{E}\left[g\left(X_{T}\right)\right]\right)^{2}\right] \leqslant \mathrm{TOL}^{2}
$$

and

$$
\operatorname{Cost}\left(\mathcal{A}_{\mathcal{M L}}\right)= \begin{cases}\mathcal{O}\left(\mathrm{TOL}^{-2}\right), & \text { if } \beta>\gamma \\ \mathcal{O}\left(\mathrm{TOL}^{-2} \log (\mathrm{TOL})^{2}\right), & \text { if } \beta=\gamma \\ \mathcal{O}\left(\mathrm{TOL}^{-2+\frac{\beta-\gamma}{\alpha}}\right), & \text { if } \beta<\gamma\end{cases}
$$


In comparison, the computational cost of achieving the goal (7) with single-level $\mathrm{MC}$ is $\mathcal{O}\left(\mathrm{TOL}^{-2-\gamma / \alpha}\right)$. Theorem 1.1 thus shows that for any problem with $\beta>0$, MLMC will asymptotically be more efficient than single-level MC. Furthermore, the performance gain of MLMC over MC is particularly apparent in settings where $\beta \geqslant \gamma$. The latter property is linked to the contributions of this work. In low-regularity SDE problems, e.g., Example 4.2 below and 35, 1], the uniform time-stepping Euler-Maruyama results in convergence rates for which $\beta<\gamma$. More sophisticated integrators can preserve rates such that $\beta \geqslant \gamma$.

Remark 1.1. Similar accuracy vs. complexity results to Theorem 1.1, requiring slightly stronger moment bounds, have also been derived for the approximation goal (2) in the asymptotic setting when TOL $\downarrow 0$, cf. [16, 5].

1.2.4. MSE A Posteriori Adaptive Time-Stepping. In general, adaptive time-stepping algorithms seek to fulfill one of two equivalent goals 22:

(B.1) Provided a computational budget $N$ and a norm $\|\cdot\|$, determine the possibly non-uniform mesh, which minimizes the error $\left\|g\left(X_{T}\right)-g\left(\bar{X}_{T}\right)\right\|$.

(B.2) Provided an error constraint $\left\|g\left(X_{T}\right)-g\left(\bar{X}_{T}\right)\right\| \leqslant$ TOL, determine the possibly non-uniform mesh, which achieves the constraint at the minimum computational cost.

Evidently, the refinement criterion of an adaptive algorithm depends on the error one seeks to minimize. In this work, we consider adaptivity goal (B.1) with the error measured in terms of the MSE. This error measure is suitable for MLMC algorithms as it often will lead to improved convergence rates, $\beta$ (since $\operatorname{Var}\left(\Delta_{\ell} g\right) \leqslant \mathrm{E}\left[\Delta_{\ell} g^{2}\right]$ ), which by Theorem 1.1 may reduce the computational cost of MLMC. In Theorem 2.1, we derive the following error expansion for the MSE of Euler-Maruyama numerical solutions of the SDE (1):

$$
\mathrm{E}\left[\left(g\left(X_{T}\right)-g\left(\bar{X}_{T}\right)\right)^{2}\right]=\mathrm{E}\left[\sum_{n=0}^{N-1} \bar{\rho}_{n} \Delta t_{n}^{2}+o\left(\Delta t_{n}^{2}\right)\right],
$$

where the error density, $\bar{\rho}_{n}$, is a function of the local error and sensitivities from the dual solution of the SDE problem, as defined in (26). The error expansion (9) is an a posteriori error estimate for the MSE, and in our adaptive algorithm, the mesh is refined by equilibration of the expansion's error indicators

$$
\bar{r}_{n}:=\bar{\rho}_{n} \Delta t_{n}^{2}, \quad \text { for } \quad n=0,1, \ldots, N-1 .
$$

1.2.5. An MSE Adaptive MLMC Algorithm. Using the described MSE adaptive algorithm, we construct an MSE adaptive MLMC algorithm in Section 3. The MLMC algorithm generates SDE realizations, $\left\{\bar{X}_{T}^{\{\ell\}}\right\}_{\ell}$, on a hierarchy of pathwise adaptively refined meshes, $\left\{\Delta t^{\{\ell\}}\right\}_{\ell}$. The meshes are nested, i.e., for all realizations $\omega \in \Omega$,

$$
\Delta t^{\{0\}}(\omega) \subset \Delta t^{\{1\}}(\omega) \subset \ldots \Delta t^{\{\ell\}}(\omega) \subset \ldots,
$$

with the constraint that the number of time steps in $\Delta t^{\{\ell\}},\left|\Delta t^{\{\ell\}}\right|$, is bounded by $2 N_{\ell}$ :

$$
\left|\Delta t^{\{\ell\}}\right|<2 N_{\ell}=2^{\ell+2} N_{-1} .
$$

Here, $N_{-1}$ denotes the pre-initial number of time steps; it is a bounded integer set in advance of the computations. This corresponds to the hierarchy setup for the uniform time-stepping MLMC algorithm in Section 1.2.2.

The potential efficiency gain of adaptive MLMC is experimentally illustrated in this work using the drift blow-up problem

$$
d X_{t}=\frac{r X_{t}}{|t-\xi|^{p}} d t+\sigma X_{t} d W_{t}, \quad X_{0}=1 .
$$

This problem is addressed in Example 4.2 for the three different singularity exponents $p=1 / 2,2 / 3$ and $3 / 4$, with a pathwise, random singularity point $\xi \sim U(1 / 4,3 / 4)$, an observable $g(x)=x$, and a final time $T=1$. For the given singularity exponents, we observe experimental deteriorating convergence rates, $\alpha=(1-p)$ and $\beta=2(1-p)$, for the uniform time-stepping Euler-Maruyama 
integrator, while for the adaptive time-step Euler-Maruyama we observe $\alpha \approx 1$ and $\beta \approx 1$. Then, as predicted by Theorem 1.1, we also observe an order of magnitude difference in computational cost between the two algorithms (cf. Table 1).

TABLE 1. Observed computational cost - disregarding $\log (\mathrm{TOL})$ multiplicative factors of finite order - for the drift blow-up study in Example 4.2 .

\begin{tabular}{cll}
\hline & \multicolumn{2}{c}{ Observed computational cost } \\
Singularity exponent $p$ & Adaptive MLMC & Uniform MLMC \\
\hline $1 / 2$ & $\mathrm{TOL}^{-2}$ & $\mathrm{TOL}^{-2}$ \\
$2 / 3$ & $\mathrm{TOL}^{-2}$ & $\mathrm{TOL}^{-3}$ \\
$3 / 4$ & $\mathrm{TOL}^{-2}$ & $\mathrm{TOL}^{-4}$ \\
\hline
\end{tabular}

1.2.6. Earlier Works on Adaptivity for SDE. Gaines' and Lyons' work 7 is one of the seminal contributions on adaptive algorithms for SDE. They present an algorithm that seeks to minimize the pathwise error of the mean and variation of the local error conditioned on the $\sigma$-algebra generated by (i.e., the values at which the Wiener path has been evaluated in order to numerically integrate the SDE realization) $\left\{W_{t_{n}}\right\}_{n=1}^{N}$. The method may be used in combination with different numerical integration methods, and an approach to approximations of potentially needed Levy areas is proposed, facilitated by a binary tree representation of the Wiener path realization at its evaluation points. As for a posteriori adaptive algorithms, the error indicators in Gaines' and Lyons' algorithm are given by products of local errors and weight terms, but, unlike in a posteriori methods, the weight terms are computed from a priori estimates, making their approach a hybrid one.

Szepessy et al. 31 introduced a posteriori weak error based adaptivity for the Euler-Maruyama algorithm with numerically computable error indicator terms. Their development of weak error adaptivity took inspiration from Talay and Tubaro's seminal work [33, where an error expansion for the weak error was derived for the Euler-Maruyama algorithm when uniform time steps were used. In [16, Szepessy et al.'s weak error adaptive algorithm was used in the construction of a weak error adaptive MLCM algorithm. To the best of our knowledge, the present work is the first on MSE a posteriori adaptive algorithms for SDE both in the MC- and MLMC setting.

Among other adaptive algorithms for SDE, many have refinement criterions based only or primarily on estimates of the local error. For example in [17, where the step-size depends on the size of the diffusion coefficient for a MSE Euler-Maruyama adaptive algorithm; in [23], the step-size is controlled by the variation in the size of the drift coefficient in the constructed EulerMaruyama adaptive algorithm, which preserves the long-term ergodic behavior of the true solution for many SDE problems; and in [19, a local error based adaptive Milstein algorithm is developed for solving multi-dimensional chemical Langevin equations.

\section{Derivation of the MSE A Posteriori Adaptive Algorithm}

In this section, we construct an MSE a posteriori adaptive algorithm for SDE whose realizations are numerically integrated by the Euler-Maruyama algorithm (3). Our goal is, in rough terms, to obtain an algorithm for solving the SDE problem (1) that for a fixed number of intervals $N$, determines the time-stepping, $\Delta t_{0}, \Delta t_{1}, \ldots, \Delta t_{N-1}$ such that the MSE, $\mathrm{E}\left[\left(g\left(\bar{X}_{T}\right)-g\left(X_{T}\right)\right)^{2}\right]$ is minimized. That is,

$$
\mathrm{E}\left[\left(g\left(\bar{X}_{T}\right)-g\left(X_{T}\right)\right)^{2}\right] \rightarrow \min !, \quad N \quad \text { given }
$$

The derivation of our adaptive algorithm consists of two steps. First, an error expansion for the MSE is presented in Theorem 2.1. Based on the error expansion, we thereafter construct a mesh refinement algorithm. At the end of the section, we apply the adaptive algorithm to a few example problems. 
2.1. The Error Expansion. Let us now present a leading-order error expansion for the MSE (11) of the SDE problem (1) in the one-dimensional (1D) setting, i.e., when $X_{t} \in \mathbb{R}$ and the drift and diffusion coefficients are respectively of the form $a:[0, T] \times \mathbb{R} \rightarrow \mathbb{R}$ and $b:[0, T] \times \mathbb{R} \rightarrow \mathbb{R}$. An extension of the MSE error expansion to multi-dimensions is given in Appendix A.3. To state the error expansion Theorem, some notation is needed. Let $X_{s}^{x, t}$ denote the solution of the SDE (1) at time $s \geqslant t$, when the initial condition is $X_{t}=x$ at time $t$, i.e.,

$$
X_{s}^{x, t}:=x+\int_{t}^{s} a\left(u, X_{u}\right) d u+\int_{t}^{s} b\left(u, X_{u}\right) d W_{u}, \quad s \in[t, T],
$$

and in light of this notation, $X_{t}$ is shorthand for $X_{t}^{x_{0}, 0}$. For a given observable $g$, the payoff-of-flow map function is defined by $\varphi(t, x)=g\left(X_{T}^{x, t}\right)$. We also make use of the following function space notation

$$
\begin{aligned}
& C(U):=\{f: U \rightarrow \mathbb{R} \mid f \text { is continuous }\}, \\
& C_{b}(U):=\{f: U \rightarrow \mathbb{R} \mid f \text { is continuous and bounded }\}, \\
& C_{b}^{k}(\mathbb{R}):=\left\{f: \mathbb{R} \rightarrow \mathbb{R} \mid f \in C(\mathbb{R}) \text { and } \frac{d^{j}}{d x^{j}} f \in C_{b}(\mathbb{R}) \text { for all integers } 1 \leqslant j \leqslant k\right\}, \\
& C_{b}^{k_{1}, k_{2}}([0, T] \times \mathbb{R}):=\{f:[0, T] \times \mathbb{R} \rightarrow \mathbb{R} \mid f \in C([0, T] \times \mathbb{R}) \text { and } \\
& \left.\quad \partial_{t}^{j_{1}} \partial_{x}^{j_{2}} f \in C_{b}([0, T] \times \mathbb{R}) \text { for all integers s.t. } j_{1} \leqslant k_{1} \text { and } 1 \leqslant j_{1}+j_{2} \leqslant k_{2}\right\} .
\end{aligned}
$$

We are now ready to present our mean square expansion result, namely,

Theorem 2.1 (1D MSE leading-order error expansion). Assume that drift and diffusion coefficients and input data of the SDE (1) fulfill

(R.1) $a, b \in C_{b}^{2,4}([0, T] \times \mathbb{R})$,

(R.2) there exists a constant $C>0$ such that

$$
|a(t, x)|^{2}+|b(t, x)|^{2} \leqslant C\left(1+|x|^{2}\right), \quad \forall x \in \mathbb{R} \text { and } \forall t \in[0, T],
$$

(R.3) $g^{\prime} \in C_{b}^{3}(\mathbb{R})$ and there exists a $k \in \mathbb{N}$ such

$$
|g(x)|+\left|g^{\prime}(x)\right| \leqslant C\left(1+|x|^{k}\right), \quad \forall x \in \mathbb{R},
$$

(R.4) for the initial data, $X_{0} \in \mathcal{F}_{0}$ and $\mathrm{E}\left[\left|X_{0}\right|^{p}\right]<\infty$ for all $p \geqslant 1$.

Assume further the mesh points $0=t_{0}<t_{1}<\ldots<t_{N}=T$

(M.1) are stopping times for which $t_{n} \in \mathcal{F}_{t_{n-1}}$ for $n=1,2, \ldots, N$,

(M.2) for all mesh realizations, there exists a deterministic integer, $\check{N}$, and a $c_{1}>0$ such that $c_{1} \check{N} \leqslant N \leqslant \check{N}$ and $a c_{2}>0$ such that $\max _{n \in\{0,1, \ldots, N-1\}} \Delta t_{n}<c_{2} \check{N}^{-1}$,

(M.3) and there exists a $c_{3}>0$ such that for all $p \in[1,8]$ and $n \in\{0,1, \ldots, \check{N}-1\}$

$$
\mathrm{E}\left[\Delta t_{n}^{2 p}\right] \leqslant c_{3}\left(\mathrm{E}\left[\Delta t_{n}^{2}\right]\right)^{p}
$$

Then, as $\check{N}$ increases,

$$
\mathrm{E}\left[\left(g\left(X_{T}\right)-g\left(\bar{X}_{T}\right)\right)^{2}\right]=\sum_{n=0}^{\check{N}-1} \mathrm{E}\left[\varphi_{x}\left(t_{n}, \bar{X}_{t_{n}}\right) \frac{\left(b_{x} b\right)^{2}}{2}\left(t_{n}, \bar{X}_{t_{n}}\right) \Delta t_{n}^{2}+o\left(\Delta t_{n}^{2}\right)\right]
$$

where we have defined $t_{n}=T$ and $\Delta t_{n}=0$ for all $n \in\{N, N+1, \ldots, \check{N}\}$. And replacing the first variation, $\varphi_{x}\left(t_{n}, \bar{X}_{n}\right)$, by the numerical approximation, $\bar{\varphi}_{x, n}$, as defined in (25), yields the following to leading order all-terms-computable error expansion:

$$
\mathrm{E}\left[\left(g\left(X_{T}\right)-g\left(\bar{X}_{T}\right)\right)^{2}\right]=\sum_{n=0}^{\check{N}-1} \mathrm{E}\left[\bar{\varphi}_{x, n}^{2} \frac{\left(b_{x} b\right)^{2}}{2}\left(t_{n}, \bar{X}_{t_{n}}\right) \Delta t_{n}^{2}+o\left(\Delta t_{n}^{2}\right)\right] .
$$


Remark 2.1. In condition (M.2) of the above theorem we have introduced $\check{N}$ to denote the deterministic upper bound for the number of time steps in all mesh realizations. Moreover, from this point on the mesh points $\left\{t_{n}\right\}_{n}$ and time steps $\left\{\Delta t_{n}\right\}_{n}$ are defined for all indices $\{0,1, \ldots, \Sigma)$ with the natural extension $t_{n}=T$ and $\Delta t_{n}=0$ for all $n \in\{N+1, \ldots, \check{N}\}$. In addition to ensuring an upper bound on the complexity of a numerical realization and that $\max _{n} \Delta t_{n} \rightarrow 0$ as $\check{N} \rightarrow \infty$, replacing the random $N$ (the smallest integer value for which $t_{N}=T$ in a given mesh) with the deterministic $\check{N}$ in the MSE error expansion (15) simplifies our proof of Theorem 2.1

Remark 2.2. For most SDE problems on which it is relevant to apply a posteriori adaptive integrators, at least one of the regularity conditions (R.1), (R.2), and (R.3) and the mesh adaptedness assumption (M.1) in Theorem 2.1 will not be fulfilled. In our adaptive algorithm, the error expansion (15) is interpreted in a formal sense and only used to facilitate the systematic construction of a mesh refinement criterion.

When applied to low-regularity SDE problems where some of the conditions (R.1), (R.2), or (R.3), do not hold, the actual leading-order term of the error expansion 15) may contain other or additional terms besides $\bar{\varphi}_{x, n}^{2} \frac{\left(b_{x} b\right)^{2}}{2}\left(t_{n}, \bar{X}_{t_{n}}\right)$ in the error density. Example 4.2 presents a problem where $a d$ hoc additional terms are added to the error density.

2.1.1. Numerical Approximation of the First Variation. The first variation of the flow map, $\varphi(t, x)$, is defined by

$$
\varphi_{x}(t, x)=\partial_{x} g\left(X_{t}^{x, t}\right)=g^{\prime}\left(X_{T}^{x, t}\right) \partial_{x} X_{T}^{x, t}
$$

and the first variation of the path itself, $\partial_{x} X_{s}^{x, t}$, is the solution of the linear SDE

$$
\begin{aligned}
d\left(\partial_{x} X_{s}^{x, t}\right) & =a_{x}\left(s, X_{s}^{x, t}\right) \partial_{x} X_{s}^{x, t} d s+b_{x}\left(s, X_{s}^{x, t}\right) \partial_{x} X_{s}^{x, t} d W_{s}, \quad s \in(t, T], \\
\partial_{x} X_{t}^{x, t} & =1 .
\end{aligned}
$$

To describe conditions under which the terms $g^{\prime}\left(X_{s}^{x, t}\right)$ and $\partial_{x} X_{s}^{x, t}$ are well defined, let us first recall that if $X_{s}^{x, t}$ solves the SDE (12) and

$$
\mathrm{E}\left[\int_{t}^{T}\left|X_{s}^{x, t}\right|^{2} d s\right]<\infty
$$

then we say that there exists a solution to the SDE; and if $\widetilde{X}_{s}^{x, t}$ is another solution of the SDE with the same initial condition, then we say the solution is pathwise unique provided that

$$
\mathrm{P}\left(\sup _{s \in[t, T]}\left|X_{s}^{x, t}-\tilde{X}_{s}^{x, t}\right|>0\right)=0 .
$$

Lemma 2.2. Assume the regularity assumptions (R.1), (R.2), (R.3), and (R.4) in Theorem 2.1 hold, and that for any fixed $t \in[0, T], x \in \mathcal{F}_{t}$ and $\mathrm{E}\left[|x|^{2 p}\right]<\infty$, for all $p \in \mathbb{N}$. Then there exist pathwise unique solutions $X_{s}^{x, t}$ and $\partial_{x} X_{s}^{x, t}$ to the respective SDE (12) and 16) for which

$$
\max \left\{\mathrm{E}\left[\sup _{s \in[t, T]}\left|X_{s}^{x, t}\right|^{2 p}\right], \mathrm{E}\left[\sup _{s \in[t, T]}\left|\partial_{x} X_{s}^{x, t}\right|^{2 p}\right]\right\}<\infty, \quad \forall p \in \mathbb{N} .
$$

Furthermore, $\varphi_{x}(t, x) \in \mathcal{F}_{T}$ and

$$
\mathrm{E}\left[\left|\varphi_{x}(t, x)\right|^{2 p}\right]<\infty, \quad \forall p \in \mathbb{N} .
$$

Proof. By writing $\left.\left(Y_{s}^{(1)}, Y_{s}^{(2)}\right):=\left(X_{s}^{x, t}, \partial_{x} X_{s}^{x, t}\right), 12\right)$ and 16 together form a system of SDE:

$$
\begin{aligned}
& d Y_{s}^{(1)}=a\left(s, Y_{s}^{(1)}\right) d s+b\left(s, Y_{s}^{(1)}\right) d W_{s} \\
& d Y_{s}^{(2)}=a_{x}\left(s, Y_{s}^{(1)}\right) Y_{s}^{(2)} d s+b_{x}\left(s, Y_{s}^{(1)}\right) Y_{s}^{(2)} d W_{s}
\end{aligned}
$$

for $s \in(t, T]$ and with initial condition $Y_{t}=(x, 1)$. By the Lipschitz continuity and the linear growth bound of this system's drift and diffusion coefficients, there exists a pathwise unique 
solution of the SDE (17) for which

$$
\mathrm{E}\left[\sup _{s \in[t, T]}\left|Y_{s}\right|^{2 p}\right]<\infty, \quad \forall p \in \mathbb{N},
$$

(cf. [22, Theorems 4.5.3 and 4.5.4 and Exercise 4.5.5]). As solutions of the Itô SDE, $X_{T}^{x, t}, \partial_{x} X_{T}^{x, t} \in$ $\mathcal{F}_{T}$, and since we assume that $g^{\prime} \in C_{b}^{3}(\mathbb{R})$, we know that $g^{\prime}$ is Borel measurable and so is the mapping $f: \mathbb{R}^{2} \rightarrow \mathbb{R}$ defined by $f(x, y)=x y$. From this we conclude that $\varphi_{x}(x, t)=$ $f\left(g^{\prime}\left(X_{T}^{x, t}\right), \partial_{x} X_{T}^{x, t}\right) \in \mathcal{F}_{T}$ and, by A.2 , Hölder's and Minkowski's inequalities, that for any $p \in \mathbb{N}$,

$$
\mathrm{E}\left[\left|\varphi_{x}(t, x)\right|^{2 p}\right] \leqslant C \sqrt{\mathrm{E}\left[\left(1+\left|X_{T}^{x, t}\right|^{k}\right)^{4 p}\right] \mathrm{E}\left[\left|\partial_{x} X_{T}^{x, t}\right|^{4 p}\right]}<\infty .
$$

To obtain an all-terms-computable error expansion in Theorem 2.1, which will be needed to construct an a posteriori adaptive algorithm, the first variation of the flow map, $\varphi_{x}$, is approximated by the first variation of the Euler-Maruyama numerical solution,

$$
\bar{\varphi}_{x, n}:=g^{\prime}\left(\bar{X}_{T}\right) \partial_{\bar{X}_{t_{n}}} \bar{X}_{T}
$$

Here, for $k>n, \partial_{\bar{X}_{t_{n}}} \bar{X}_{t_{k}}$ is the solution of the Euler-Maruyama scheme

$$
\left(\partial_{\bar{X}_{t_{n}}} \bar{X}\right)_{t_{j+1}}=\left(\partial_{\bar{X}_{t_{n}}} \bar{X}\right)_{t_{j}}+a_{x}\left(t_{j}, \bar{X}_{t_{j}}\right)\left(\partial_{\bar{X}_{t_{n}}} \bar{X}\right)_{t_{j}} \Delta t_{j}+b_{x}\left(t_{j}, \bar{X}_{t_{j}}\right)\left(\partial_{\bar{X}_{t_{n}}} \bar{X}\right)_{t_{j}} \Delta W_{j}
$$

for $j=n, n+1, \ldots k-1$ and with the initial condition $\partial_{\bar{X}_{t_{n}}} \bar{X}_{t_{n}}=1$, which is coupled to the numerical solution of the SDE, $\bar{X}_{t_{j}}$.

Lemma 2.3. If the assumptions (R.1), (R.2), (R.3), (R.4), (M.1) and (M.2) in Theorem 2.1 hold, then the numerical solution $\bar{X}$ of (3) converges in mean square sense to the solution of the $S D E$ (1),

$$
\max _{1 \leqslant n \leqslant \check{N}}\left(\mathrm{E}\left[\left|\bar{X}_{t_{n}}-X_{t_{n}}\right|^{2 p}\right]\right)^{1 / 2 p} \leqslant C \check{N}^{-1 / 2}
$$

and

$$
\max _{1 \leqslant n \leqslant N} \mathrm{E}\left[\left|\bar{X}_{t_{n}}\right|^{2 p}\right]<\infty, \quad \forall p \in \mathbb{N} .
$$

For any fixed $1 \leqslant n \leqslant \check{N}$, the numerical solution $\partial_{\bar{X}_{t_{n}}} \bar{X}$ of 18 converges in mean square sense to $\partial_{x} X^{X_{t_{m}}, t_{m}}$

$$
\max _{n \leqslant k \leqslant N}\left(\mathrm{E}\left[\left|\partial_{\bar{X}_{t_{n}}} \bar{X}_{t_{k}}-\partial_{x} X_{t_{k}}^{X_{t_{n}}, t_{n}}\right|^{2 p}\right]\right)^{1 / 2 p} \leqslant C \check{N}^{-1 / 2}
$$

and

$$
\max _{n \leqslant k \leqslant N} \mathrm{E}\left[\left|\partial_{\bar{X}_{t_{n}}} \bar{X}_{t_{k}}\right|^{2 p}\right]<\infty, \quad \forall p \in \mathbb{N} .
$$

Furthermore, $\bar{\varphi}_{x, n} \in \mathcal{F}_{T}$ and

$$
\mathrm{E}\left[\left|\bar{\varphi}_{x, n}\right|^{2 p}\right]<\infty, \quad \forall p \in \mathbb{N}
$$

Proof. The system $\bar{Y}_{t_{k}}:=\left(\bar{X}_{t_{k}}, \partial_{\bar{X}_{t_{n}}} \bar{X}_{t_{k}}\right)$ provides solutions approximating the SDE (17) that are generated by the Euler-Maruyama scheme

$$
\begin{aligned}
& Y_{t_{k+1}}^{(1)}=Y_{t_{k}}^{(1)}+a\left(t_{k}, Y_{t_{k}}^{(1)}\right) \Delta t_{k}+b\left(t_{k}, Y_{t_{k}}^{(1)}\right) \Delta W_{k}, \\
& Y_{t_{k+1}}^{(2)}=Y_{t_{k}}^{(2)}+a_{x}\left(t_{k}, Y_{t_{k}}^{(1)}\right) Y_{t_{k}}^{(2)} \Delta t_{k}+b_{x}\left(t_{k}, Y_{t_{k}}^{(1)}\right) Y_{t_{k}}^{(2)} \Delta W_{k},
\end{aligned}
$$

for $k \geqslant n$ and with initial condition $\bar{Y}_{t_{n}}=\left(\bar{X}_{t_{n}}, 1\right)$. The assumptions of [22, Theorem 10.6.3 and the remark following it] are fulfilled for the SDE (17), which implies the strong convergence of $\bar{Y}$ to $Y$ and that the inequalities $(19),(20),(21)$, and $(22)$ hold. That $\bar{\varphi}_{x, n} \in \mathcal{F}_{T}$ and that inequality (23) holds can be shown by a similar argument as in the proof of Lemma 2.2 
From the SDE (24), it is clear that

$$
\partial_{\bar{X}_{n}} \bar{X}_{T}=\prod_{k=n}^{N-1}\left(1+a_{x}\left(t_{k}, \bar{X}_{t_{k}}\right) \Delta t_{k}+b_{x}\left(t_{k}, \bar{X}_{t_{k}}\right) \Delta W_{k}\right),
$$

and this implies that $\bar{\varphi}_{x, n}$ solves the backward scheme

$$
\bar{\varphi}_{x, n}=c_{x}\left(t_{n}, \bar{X}_{t_{n}}\right) \bar{\varphi}_{x, n+1}, \quad n=N-1, N-2, \ldots, 0
$$

with the initial condition $\bar{\varphi}_{x, N}=g^{\prime}\left(\bar{X}_{T}\right)$ and the shorthand notation

$$
c\left(t_{n}, \bar{X}_{t_{n}}\right):=\bar{X}_{t_{n}}+a\left(t_{n}, \bar{X}_{t_{n}}\right) \Delta t_{n}+b\left(t_{n}, \bar{X}_{t_{n}}\right) \Delta W_{n}
$$

The backward scheme 25) is convenient from a computational perspective since it implies that the set of points, $\left\{\bar{\varphi}_{x, n}\right\}_{n=0}^{N}$, can be computed at the same cost as that of one-path realization, $\left\{\bar{X}_{t_{n}}\right\}_{n=0}^{N}$, which can be verified as follows

$$
\begin{aligned}
\bar{\varphi}_{x, n} & =g^{\prime}\left(\bar{X}_{T}\right) \prod_{k=n}^{N-1} c_{x}\left(t_{k}, \bar{X}_{t_{k}}\right) \\
& =c_{x}\left(t_{n}, \bar{X}_{t_{n}}\right) g^{\prime}\left(\bar{X}_{T}\right) \prod_{k=n+1}^{N-1} c_{x}\left(t_{k}, \bar{X}_{t_{k}}\right) \\
& =c_{x}\left(t_{n}, \bar{X}_{t_{n}}\right) g^{\prime}\left(\bar{X}_{T}\right) \partial_{t_{n+1}} \bar{X}_{T} \\
& =c_{x}\left(t_{n}, \bar{X}_{t_{n}}\right) \bar{\varphi}_{x, n+1} .
\end{aligned}
$$

2.2. The Adaptive Algorithm. Having derived computable expressions for all terms in the error expansion, we next introduce the error density

$$
\bar{\rho}_{n}:=\bar{\varphi}_{x, n}^{2} \frac{\left(b_{x} b\right)^{2}}{2}\left(t_{n}, \bar{X}_{t_{n}}\right), \quad n=0,1, \ldots, N-1,
$$

and, for representing the numerical solution's error contribution from the time interval $\left(t_{n}, t_{n+1}\right)$, the error indicators

$$
\bar{r}_{n}:=\bar{\rho}_{n} \Delta t_{n}^{2}, \quad n=0,1, \ldots, N-1 .
$$

The error expansion (15) may then be written as

$$
\mathrm{E}\left[\left(g\left(X_{T}\right)-g\left(\bar{X}_{T}\right)\right)^{2}\right]=\sum_{n=0}^{\check{N}-1} \mathrm{E}\left[\bar{r}_{n}+o\left(\Delta t_{n}^{2}\right)\right] .
$$

The final goal of the adaptive algorithm is minimization of the leading order of the MSE in (28), namely, $\mathrm{E}\left[\sum_{n=0}^{N-1} \bar{r}_{n}\right]$, which (for each realization) is approached by minimization of the error expansion realization $\sum_{n=0}^{N-1} \bar{r}_{n}$. An approximately optimal choice for the refinement procedure can be derived by introducing the Lagrangian

$$
\mathcal{L}(\Delta t, \lambda)=\int_{0}^{T} \rho(s) \Delta t(s) d s+\lambda\left(\int_{0}^{T} \frac{1}{\Delta t(s)} d s-\check{N}\right),
$$

for which we seek to minimize the pathwise squared error

$$
\left(g\left(X_{T}\right)-g\left(\bar{X}_{T}\right)\right)^{2}=\int_{0}^{T} \rho(s) \Delta t(s) d s
$$

under the constraint that

$$
\int_{0}^{T} \frac{1}{\Delta t(s)} d s=\check{N}
$$

for a fixed number of time steps, $\check{N}$, and the implicit constraint that the error indicators are equilibrated,

$$
\bar{r}_{n}=\bar{\rho}_{n} \Delta t_{n}^{2}=\frac{\left(g\left(X_{T}\right)-g\left(\bar{X}_{T}\right)\right)^{2}}{\check{N}}, \quad n=0,1, \ldots, \check{N}-1 .
$$


Minimizing 29) yields

$$
\Delta t_{n}=\sqrt{\frac{\left(g\left(X_{T}\right)-g\left(\bar{X}_{T}\right)\right)^{2}}{\check{N} \rho\left(t_{n}\right)}} \text { and } \mathrm{MSE}_{\text {adaptive }} \leqslant \frac{1}{\check{N}} \mathrm{E}\left[\left(\int_{0}^{T} \sqrt{\rho(s)} d s\right)^{2}\right] \text {, }
$$

where the above inequality follows from using Hölder's inequality,

$$
\begin{aligned}
\mathrm{E}\left[\left(g\left(X_{T}\right)-g\left(\bar{X}_{T}\right)\right)^{2}\right] & =\frac{1}{\sqrt{\check{N}}} \mathrm{E}\left[\left|g\left(X_{T}\right)-g\left(\bar{X}_{T}\right)\right| \int_{0}^{T} \sqrt{\rho(s)} d s\right] \\
& \leqslant \frac{1}{\sqrt{\check{N}}} \sqrt{\mathrm{E}\left[\left|g\left(X_{T}\right)-g\left(\bar{X}_{T}\right)\right|^{2}\right]} \sqrt{\mathrm{E}\left[\left(\int_{0}^{T} \sqrt{\rho(s)} d s\right)^{2}\right]} .
\end{aligned}
$$

In comparison, we notice that if a uniform mesh is used, the MSE becomes

$$
\mathrm{MSE}_{\text {uniform }}=\frac{T}{\check{N}} \mathrm{E}\left[\int_{0}^{T} \rho(s) d s\right]
$$

A consequence of observations (31) and 32 is that for many low-regularity problems, for instance, if $\rho(s)=s^{-p}$ with $p \in[1,2)$, adaptive time-stepping Euler-Maruyama methods may produce more accurate solutions (measured in the MSE) than are obtained using the uniform time-stepping Euler-Maruyama method under the same computational budget constraints.

2.2.1. Mesh Refinement Strategy. To equilibrate the error indicators (30), we propose an iterative mesh refinement strategy to identify the largest error indicator and then refining the corresponding time step by halving it. To compute the error indicators prior to refinement, the algorithm first computes the numerical SDE solution, $\bar{X}_{t_{n}}$, and the corresponding first variation $\bar{\varphi}_{x, n}$ (using equations (3) and (25) respectively) on the initial mesh, $\Delta t^{\{0\}}$. Thereafter, the error indicators $\bar{r}_{n}$ are computed by Equation (27) and the mesh is refined a prescribed number of times, $N_{\text {refine, as }}$ follows:

(C.1) Find the largest error indicator

$$
n^{*}:=\arg \max _{n} \bar{r}_{n}
$$

and refine the corresponding time step by halving

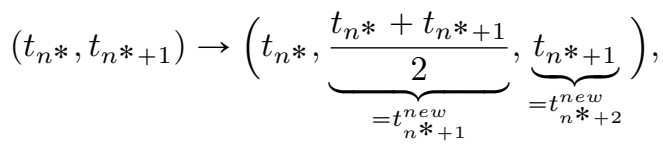

and increment the number of refinements by one.

(C.2) Update the values of the error indicators, either by recomputing the whole problem or locally by interpolation, cf. Section 2.2 .3 .

(C.3) Go to step (C.4) if $N_{\text {refine }}$ mesh refinements have been made; otherwise, return to step (C.1).

(C.4) (Postconditioning) Do a last sweep over the mesh and refine by halving every time step that is strictly larger than $\Delta t_{\max }$, where $\Delta t_{\max }=\mathcal{O}\left(\check{N}^{-1}\right)$ denotes the maximum allowed step size.

The postconditioning step (C.4) ensures that all time steps become infinitesimally small as the number of time steps $N \rightarrow \infty$ with such a rate of decay that condition (M.2) in Theorem 2.1 holds and is thereby one of the necessary conditions from Lemma 2.3 to ensure strong convergence for the numerical solutions of the MSE adaptive Euler-Maruyama algorithm. However, the strong convergence result should primarily be interpreted as a motivation for introducing the postconditioning step (C.4) since Theorem 2.1 s assumption (M.1), namely that the mesh points are stopping times for which $t_{n} \in \mathcal{F}_{t_{n-1}}$, will not hold in general for our adaptive algorithm. 
2.2.2. Wiener Path Refinements. When a time step is refined, as described in (34), the Wiener path must be refined correspondingly. The value of the Wiener path at the midpoint between $W_{t_{n} *}$ and $W_{t_{n *+1}}$ can be generated by Brownian bridge interpolation,

$$
W_{t_{n *+1}^{n e w}}=\frac{W_{t_{n *}}+W_{t_{n *+1}}}{2}+\xi \frac{\sqrt{\Delta t_{n *}}}{2}
$$

where $\xi \sim N(0,1)$, cf. [27. See Figure 1 for an illustration of Brownian bridge interpolation applied to numerical solutions of an Ornstein-Uhlenbeck SDE.

2.2.3. Updating the Error Indicators. After the refinement of an interval, $\left(t_{n^{*}}, t_{n^{*}+1}\right)$, and its Wiener path, error indicators must also be updated before moving on to determine which interval is next in line for refinement. There are different ways of updating error indicators. One expensive but more accurate option is to recompute the error indicators completely by first solving the forward problem (3) and the backward problem (25). A less costly but also less accurate alternative is to update only the error indicators locally at the refined time step by one forward and backward numerical solution step, respectively:

$$
\begin{aligned}
\bar{X}_{t_{n *+1}}^{n e w} & =\bar{X}_{t_{n *}}+a\left(t_{n *}, \bar{X}_{t_{n *}}\right) \Delta t_{n^{*}}^{n e w}+b\left(t_{n *}, \bar{X}_{t_{n *}}\right) \Delta W_{n *}^{n e w}, \\
\bar{\varphi}_{x, n^{*}+1}^{n e w} & =c_{x}\left(t_{n *}^{n e w}, \bar{X}_{t_{n *}^{n e w}}\right) \bar{\varphi}_{x, n^{*}+1} .
\end{aligned}
$$

Thereafter, we compute the resulting error density, $\bar{\rho}_{n *+1}^{n e w}$, by Equation (26), and finally update the error locally by

$$
\bar{r}_{n *}=\bar{\rho}_{n *}\left(\Delta t_{n *}^{n e w}\right)^{2}, \quad \bar{r}_{n *+1}=\bar{\rho}_{n *+1}^{n e w}\left(\Delta t_{n *+1}^{n e w}\right)^{2} .
$$

As a compromise between cost and accuracy, we here propose the following mixed approach to updating error indicators post refinement: With $N_{\text {refine }}$ denoting the prescribed number of refinement iterations of the input mesh, let all error indicators be completely recomputed every $\tilde{N}=\mathcal{O}\left(\log \left(N_{\text {refine }}\right)\right)$-th iteration, whereas for the remaining $N_{\text {refine }}-\tilde{N}$ iterations, only local updates of the error indicators are computed. Following this approach, the computational cost of refining a mesh holding $N$ time steps into a mesh of $2 N$ time steps becomes $\mathcal{O}\left(N \log (N)^{2}\right)$. Observe that the asymptotically dominating cost is to sort the mesh's error indicators $\mathcal{O}(\log (N))$ times. To anticipate the computational cost for the MSE adaptive MLMC algorithm, this implies that the cost of generating an MSE adaptive realization pair is $\operatorname{Cost}\left(\Delta_{\ell} g\right)=\mathcal{O}\left(\ell^{2} 2^{\ell}\right)$.

2.2.4. Pseudocode. The mesh refinement and the computation of error indicators are presented in Algorithms 1 and 2, respectively. 

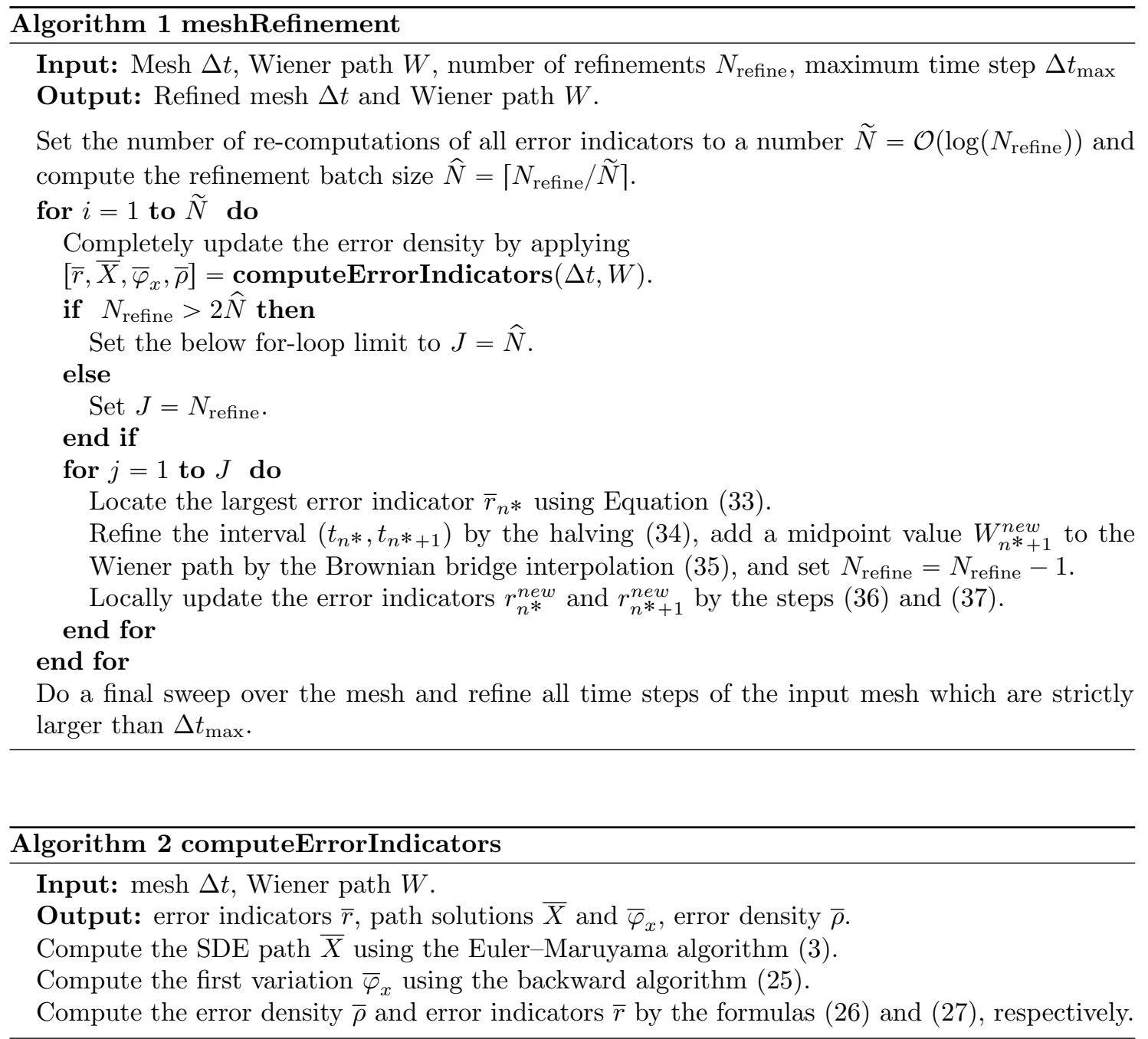

2.3. Numerical Examples. To illustrate the procedure for computing error indicators and the performance of the adaptive algorithm, we now present four SDE example problems. To keep matters relatively elementary, the dual solutions, $\varphi_{x}(t)$, for these examples are derived not from a posteriori but a priori analysis. This approach results in adaptively generated mesh points which for all problems in this section will contain mesh points which are stopping times for which $t_{n} \in \mathcal{F}_{t_{n-1}}$ for all $n \in\{1,2, \ldots, N\}$. In Examples 2.1 2.2 and 2.3 it is straightforward to verify that the other assumptions of the respective single- and multi-dimensional MSE error expansions of Theorems 2.1 and A.2 hold, meaning that the adaptive approach produces numerical solutions whose MSE to leading order are bounded by the respective error expansions (14) and (68).

Example 2.1. We consider the classical geometric Brownian motion problem

$$
d X_{t}=X_{t} d t+X_{t} d W_{t}, \quad X_{0}=1,
$$

for which we seek to minimize the MSE

$$
\mathrm{E}\left[\left(X_{T}-\bar{X}_{T}\right)^{2}\right]=\min !, \quad N \text { given, }
$$

at the final time, $T=1$, (cf. the goal (B.1)). One may derive that the dual solution of this problem is of the form

$$
\varphi_{x}\left(X_{t}, t\right)=\partial_{X_{t}} X_{T}^{X_{t}, t}=\frac{X_{T}}{X_{t}},
$$


which leads to the error density

$$
\rho(t)=\frac{\left(b_{x} b\right)^{2}\left(X_{t}, t\right)\left(\varphi_{x}\left(X_{t}, t\right)\right)^{2}}{2}=\frac{X_{T}^{2}}{2} .
$$

We conclude that uniform time-stepping is optimal. A further reduction of the MSE could be achieved by allowing the number of time steps to depend on the magnitude of $X_{T}^{2}$ for each realization. This is however outside the scope of the considered refinement goal (B.1), where we assume the number of time steps, $N$, is fixed for all realizations and would be possible only to a very weak degree under the slight generalization of (B.1) given in assumption (M.2) of Theorem 2.1.

Example 2.2. Our second example is the two-dimensional (2D) SDE problem

$$
\begin{aligned}
d W_{t} & =1 d W_{t}, & W_{0} & =0, \\
d X_{t} & =W_{t} d W_{t}, & X_{0} & =0 .
\end{aligned}
$$

Here, we seek to minimize the MSE $\mathrm{E}\left[\left(X_{T}-\bar{X}_{T}\right)^{2}\right]$ for the observable

$$
X_{T}=\int_{0}^{T} W_{t} d W_{t}
$$

at the final time $T=1$. With the diffusion matrix represented by

$$
b\left(\left(W_{t}, X_{t}\right), t\right)=\left[\begin{array}{c}
1 \\
W_{t}
\end{array}\right],
$$

and observing that

$$
\partial_{X_{t}} X_{T}^{X_{t}, t}=\partial_{X_{t}}\left(X_{t}+\int_{t}^{T} W_{s} d W_{s}\right)=1
$$

it follows from the error density in multi-dimensions in Equation 66 that $\rho(t)=\frac{1}{2}$. We conclude that uniform time-stepping is optimal for this problem as well.

Example 2.3. Next, we consider the three-dimensional (3D) SDE problem

$$
\begin{aligned}
d W_{t}^{(1)} & =1 d W_{t}^{(1)}, & W_{0}^{(1)} & =0, \\
d W_{t}^{(2)} & =1 d W_{t}^{(2)}, & W_{0}^{(2)} & =0, \\
d X_{t} & =W_{t}^{(1)} d W_{t}^{(2)}-W_{t}^{(2)} d W_{t}^{(1)}, & X_{0} & =0,
\end{aligned}
$$

where $W_{t}^{(1)}$ and $W_{t}^{(2)}$ are independent Wiener processes. Here, we seek to minimize the MSE $\mathrm{E}\left[\left(X_{T}-\bar{X}_{T}\right)^{2}\right]$ for the Levy area observable

$$
X_{T}=\int_{0}^{T}\left(W_{t}^{(1)} d W_{t}^{(2)}-W_{t}^{(2)} d W_{t}^{(1)}\right),
$$

at the final time, $T=1$. Representing the diffusion matrix by

$$
b\left(\left(W_{t}, X_{t}\right), t\right)=\left[\begin{array}{cc}
1 & 0 \\
0 & 1 \\
-W_{t}^{(1)} & W_{t}^{(2)}
\end{array}\right],
$$

and observing that

$$
\partial_{X_{t}} X_{T}^{X_{t}, t}=\partial_{X_{t}}\left(X_{t}+\int_{t}^{T}\left(W_{s}^{(1)} d W_{s}^{(2)}-W_{s}^{(2)} d W_{s}^{(1)}\right),\right)=1,
$$

it follows from Equation $(66)$ that $\rho(t)=1$. We conclude that uniform time-stepping is optimal for computing Levy areas. 
Example 2.4. As the last example, we consider the 2D SDE

$$
\begin{array}{ll}
d W_{t}=1 d W_{t}, & W_{0}=0, \\
d X_{t}=3\left(W_{t}^{2}-t\right) d W_{t}, & X_{0}=0 .
\end{array}
$$

We seek to minimize the MSE (38) at the final time $T=1$. For this problem, it may be shown by Itô calculus that the pathwise exact solution is $X_{T}=W_{T}^{3}-3 W_{T} T$. Representing the diffusion matrix by

$$
b\left(\left(W_{t}, X_{t}\right), t\right)=\left[\begin{array}{c}
1 \\
3\left(W_{t}^{2}-t\right)
\end{array}\right]
$$

equation (66) implies that $\rho(t)=18 W_{t}^{2}$. This motivates the use of discrete error indicators, $\bar{r}_{n}=18 W_{t_{n}}^{2} \Delta t_{n}^{2}$, in the mesh refinement criterion. For this problem, we may not directly conclude that the error expansion (68) holds since the diffusion coefficient does not fulfill the assumption in Theorem A.2. Although we will not include the details here, it is easy to derive that $\partial_{x}^{j} X_{T}^{x, t}=0$ for all $j>1$ and to prove that the MSE leading-order error expansion also holds for this particular problem by following the steps of the proof of Theorem 2.1. In Figure 2, we compare the uniform and adaptive time-stepping Euler-Maruyama algorithms in terms of MSE vs. the number of time steps, $N$. Estimates for the MSE for both algorithms are computed by MC sampling using $M=10^{6}$ samples. This is a sufficient sample size to render the MC estimates' statistical error negligible. For the adaptive algorithm, we have used the following input parameter in Algorithm 1 uniform input mesh, $\Delta t$, with step size $2 / N$ (and $\Delta t_{\max }=2 / N$ ). The number of refinements is set to $N_{\text {refine }}=N / 2$. We observe that the algorithms have approximately equal convergence rates, but, as expected, the adaptive algorithm is slightly more accurate than the uniform time-stepping algorithm.

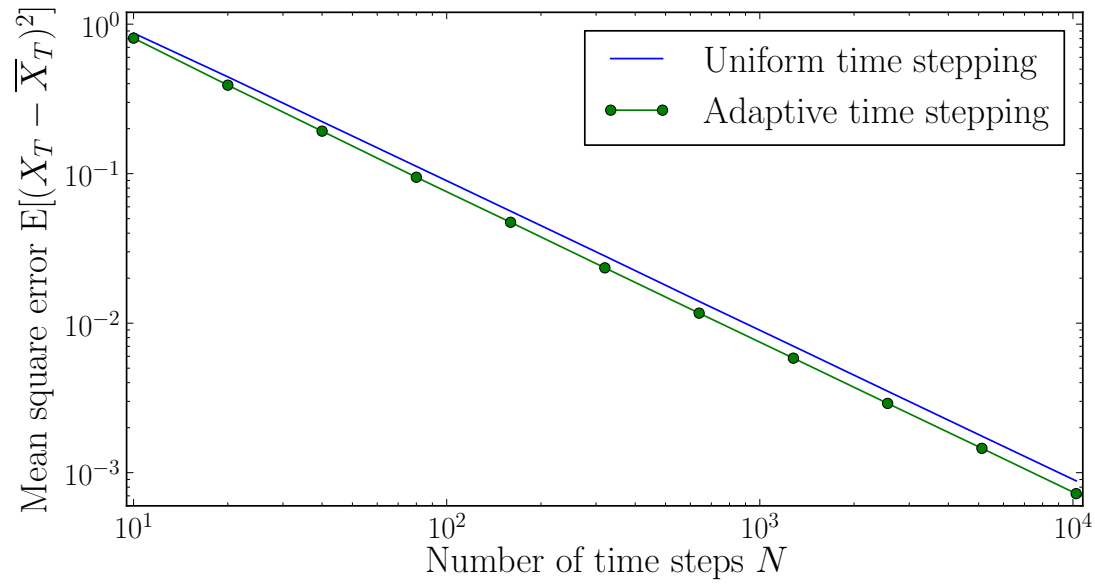

FIGURE 2. Comparison of the performance of uniform and adaptive time-stepping Euler-Maruyama numerical integration for Example 2.4 in terms of MSE vs. number of time steps.

\section{Extension of the Adaptive Algorithm to the Multilevel Setting}

In this section, we incorporate the MSE adaptive time-stepping algorithm presented in the preceding section into an MSE adaptive MLMC algorithm for weak approximations. First, we shortly recall the approximation goal and important concepts for the MSE adaptive MLMC algorithm, such as the structure of the adaptive mesh hierarchy and MLMC error control. Thereafter, the MLMC algorithm is presented in pseudocode form. 
3.1. Notation and Objective. For a tolerance, TOL $>0$, and confidence, $0<1-\delta<1$, we recall that our objective is to construct an adaptive time-stepping MLMC estimator, $\mathcal{A}_{\mathcal{M} \mathcal{L}}$, which meets the approximation constraint

$$
\mathrm{P}\left(\left|\mathrm{E}\left[g\left(X_{T}\right)\right]-\mathcal{A}_{\mathcal{M L}}\right| \leqslant \mathrm{TOL}\right) \geqslant 1-\delta .
$$

We denote the multilevel estimator by

$$
\mathcal{A}_{\mathcal{M L}}:=\sum_{\ell=0}^{L} \underbrace{\sum_{\ell}^{M_{\ell}} \frac{\Delta_{\ell} g\left(\omega_{i, \ell}\right)}{M_{\ell}}}_{=: \mathcal{A}\left(\Delta_{\ell} g ; M_{\ell}\right)},
$$

where

$$
\Delta_{\ell} g(\omega):= \begin{cases}g\left(\bar{X}_{T}^{\{0\}}(\omega)\right), & \text { if } \ell=0, \\ g\left(\bar{X}_{T}^{\{\ell\}}(\omega)\right)-g\left(\bar{X}_{T}^{\{\ell-1\}}(\omega)\right), & \text { else. }\end{cases}
$$

Section 1.2 .5 presents further details on MLMC notation and parameters.

3.1.1. The Mesh Hierarchy. A realization, $\Delta_{\ell} g\left(\omega_{i, \ell}\right)$, is generated on a nested pair of mesh realizations

$$
\ldots \subset \Delta t^{\{\ell-1\}}\left(\omega_{i, \ell}\right) \subset \Delta t^{\{\ell\}}\left(\omega_{i, \ell}\right) .
$$

Subsequently, mesh realizations are generated step by step from a prescribed and deterministic input mesh, $\Delta t^{\{-1\}}$, holding $N_{-1}$ uniform time steps. First, $\Delta t^{\{-1\}}$ is refined into a mesh, $\Delta t^{\{0\}}$, by applying Algorithm 1 , namely

$$
\left[\Delta t^{\{0\}}, W^{\{0\}}\right]=\text { meshRefinement }\left(\Delta t^{\{-1\}}, W^{\{-1\}}, N_{\text {refine }}=N_{-1}, \Delta t_{\max }=N_{0}^{-1}\right) .
$$

The mesh refinement process is iterated until meshes $\Delta t^{\{\ell-1\}}$ and $\Delta t^{\{\ell-1\}}$ are produced, with the last couple of iterations being

$$
\left[\Delta t^{\{\ell-1\}}, W^{\{\ell-1\}}\right]=\text { meshRefinement }\left(\Delta t^{\{\ell-2\}}, W^{\{\ell-2\}}, N_{\text {refine }}=N_{\ell-2}, \Delta t_{\max }=N_{\ell-1}^{-1}\right),
$$

and

$$
\left[\Delta t^{\{\ell\}}, W^{\{\ell\}}\right]=\text { meshRefinement }\left(\Delta t^{\{\ell-1\}}, W^{\{\ell-1\}}, N_{\text {refine }}=N_{\ell-1}, \Delta t_{\max }=N_{\ell}^{-1}\right) .
$$

The output realization for the difference $\Delta_{\ell} g\left(\omega_{i, \ell}\right)=g\left(\bar{X}_{T}^{\{\ell\}}\left(\omega_{i, \ell}\right)\right)-g\left(\bar{X}_{T}^{\{\ell-1\}}\left(\omega_{i, \ell}\right)\right)$ is thereafter generated on the output temporal mesh and Wiener path pairs, $\left(\Delta t^{\{\ell-1\}}, W^{\{\ell-1\}}\right)$ and $\left(\Delta t^{\{\ell\}}, W^{\{\ell\}}\right)$.

For later estimates of the computational cost of the MSE adaptive MLMC algorithm, it is useful to have upper bounds on the growth of the number of time steps in the mesh hierarchy, $\left\{\Delta t^{\{\ell\}}\right\}_{\ell}$, as $\ell$ increases. Letting $|\Delta t|$ denote the number of time steps in a mesh, $\Delta t$ (i.e., the cardinality of the set $\left.\Delta t=\left\{\Delta t_{0}, \Delta t_{1}, \ldots\right\}\right)$, the following bounds hold

$$
N_{\ell} \leqslant\left|\Delta t^{\{\ell\}}\right|<2 N_{\ell} \quad \forall \ell \in \mathbb{N}_{0} .
$$

The lower bound follows straightforwardly from the mesh hierarchy refinement procedure described above. To show the upper bound, notice the maximum number of mesh refinements going from a level $\ell-1$ mesh, $\Delta t^{\{\ell-1\}}$ to a level $\ell$ mesh, $\Delta t^{\{\ell\}}$ is $2 N_{\ell-1}-1$. Consequently,

$$
\begin{aligned}
\left|\Delta t^{\{\ell\}}\right| & \leqslant\left|\Delta t^{\{-1\}}\right|+\sum_{j=0}^{\ell-1} \text { Maximum number of refinements going from } \Delta t^{\{j-1\}} \text { to } \Delta t^{\{j\}} \\
& \leqslant N_{-1}+2 \sum_{j=0}^{\ell} N_{j-1}-(\ell+1)<2 N_{\ell} .
\end{aligned}
$$


Remark 3.1. For the telescoping property $\mathrm{E}\left[\mathcal{A}_{\mathcal{M L}}\right]=\mathrm{E}\left[g\left(\bar{X}_{T}^{\{\ell\}}\right)\right]$ to hold, it is not required that the adaptive mesh hierarchy is nested, but non-nested meshes make it more complicated to compute Wiener path pairs $\left(W^{\{\ell-1\}}, W^{\{\ell\}}\right)(\omega)$. In the numerical tests leading to this work, we tested both nested and non-nested adaptive meshes and found both options performing satisfactorily.

3.2. Error Control. The error control for the adaptive MLMC algorithm follows the general framework of a uniform time-stepping MLMC, but for the sake of completeness, we recall the error control framework for the setting of weak approximations. By splitting

$$
\left|\mathrm{E}\left[g\left(X_{T}\right)\right]-\mathcal{A}_{\mathcal{M L}}\right| \leqslant \underbrace{\left|\mathrm{E}\left[g\left(X_{T}\right)-g\left(\bar{X}_{T}^{\{L\}}\right)\right]\right|}_{=: \mathcal{E}_{T}}+\underbrace{\left|\mathrm{E}\left[g\left(\bar{X}_{T}^{\{L\}}\right)-\mathcal{A}_{\mathcal{M L}}\right]\right|}_{=: \mathcal{E}_{S}}
$$

and

$$
\mathrm{TOL}=\mathrm{TOL}_{\mathrm{T}}+\mathrm{TOL}_{\mathrm{S}}
$$

we seek to implicitly fulfill (39) by imposing the stricter constraints

$$
\begin{aligned}
\mathcal{E}_{T} & \leqslant \mathrm{TOL}_{\mathrm{T}}, & & \text { the time discretization error, } \\
P\left(\mathcal{E}_{S} \leqslant \mathrm{TOL}_{\mathrm{S}}\right) & \geqslant 1-\delta, & & \text { the statistical error. }
\end{aligned}
$$

3.2.1. The Statistical Error. Under the moment assumptions stated in 6], Lindeberg's version of the Central Limit Theorem yields that as TOL $\downarrow 0$,

$$
\frac{\mathcal{A}_{\mathcal{M L}}-\mathrm{E}\left[g\left(\bar{X}_{T}^{\{L\}}\right)\right]}{\sqrt{\operatorname{Var}\left(\mathcal{A}_{\mathcal{M L}}\right)}} \stackrel{D}{\rightarrow} N(0,1) .
$$

Here, $\stackrel{D}{\longrightarrow}$ denotes convergence in distribution. By construction, we have

$$
\operatorname{Var}\left(\mathcal{A}_{\mathcal{M L}}\right)=\sum_{\ell=0}^{L} \frac{\operatorname{Var}\left(\Delta_{\ell} g\right)}{M_{\ell}}
$$

This asymptotic result motivates the statistical error constraint

$$
\operatorname{Var}\left(\mathcal{A}_{\mathcal{M L}}\right) \leqslant \frac{\mathrm{TOL}_{\mathrm{S}}{ }^{2}}{C_{\mathrm{C}}{ }^{2}}
$$

where $C_{\mathrm{C}}(\delta)$ is the confidence parameter chosen such that

$$
1-\frac{1}{\sqrt{2 \pi}} \int_{-C_{\mathrm{C}}}^{C_{\mathrm{C}}} e^{-x^{2} / 2} d x=(1-\delta),
$$

for a prescribed confidence $(1-\delta)$.

Another important question is how to distribute the number of samples, $\left\{M_{\ell}\right\}_{\ell}$, on the level hierarchy such that both the computational cost of the MLMC estimator is minimized and the constraint (43) is met. Letting $C_{\ell}$ denote the expected cost of generating a numerical realization $\Delta_{\ell} g\left(\omega_{i, \ell}\right)$, the approximate total cost of generating the multilevel estimator becomes

$$
\mathcal{C}_{\mathcal{M L}}:=\sum_{\ell=0}^{L} C_{\ell} M_{\ell}
$$

An optimization of the number of samples at each level can then be found through minimization of the Lagrangian

$$
\mathcal{L}\left(M_{0}, M_{1}, \ldots, M_{L}, \lambda\right)=\lambda\left(\sum_{\ell=0}^{L} \frac{\operatorname{Var}\left(\Delta_{\ell} g\right)}{M_{\ell}}-\frac{\mathrm{TOL}_{\mathrm{S}}{ }^{2}}{C_{\mathrm{C}}{ }^{2}}\right)+\sum_{\ell=0}^{L} C_{\ell} M_{\ell},
$$

yielding

$$
M_{\ell}=\left\lceil\frac{C_{\mathrm{C}}^{2}}{\mathrm{TOL}_{\mathrm{S}}^{2}} \sqrt{\frac{\operatorname{Var}\left(\Delta_{\ell} g\right)}{C_{\ell}}} \sum_{\ell=0}^{L} \sqrt{C_{\ell} \operatorname{Var}\left(\Delta_{\ell} g\right)}\right\rceil, \quad \ell=0,1, \ldots, L
$$


Since the cost of adaptively refining a mesh, $\Delta t^{\{\ell\}}$, is $\mathcal{O}\left(N_{\ell} \log \left(N_{\ell}\right)^{2}\right)$, as noted in Section 2.2.3 the cost of generating an SDE realization, is of the same order: $C_{\ell}=\mathcal{O}\left(N_{\ell} \log \left(N_{\ell}\right)^{2}\right)$. Representing the cost by its leading-order term and disregarding the logarithmic factor, an approximation to the level-wise optimal number of samples becomes

$$
M_{\ell}=\left\lceil\frac{C_{\mathrm{C}}^{2}}{\mathrm{TOL}_{\mathrm{S}}^{2}} \sqrt{\frac{\operatorname{Var}\left(\Delta_{\ell} g\right)}{N_{\ell}}} \sum_{\ell=0}^{L} \sqrt{N_{\ell} \operatorname{Var}\left(\Delta_{\ell} g\right)}\right\rceil, \quad \ell=0,1, \ldots, L .
$$

Remark 3.2. In our MLMC implementations, the variances, $\operatorname{Var}\left(\Delta_{\ell} g\right)$, in equation 45 are approximated by sample variances. To save memory in our parallel computer implementation, the maximum permitted batch size for a set of realizations, $\left\{\Delta_{\ell} g\left(\omega_{i, \ell}\right)\right\}_{i}$, is set to 100,000 . For the initial batch consisting of $M_{\ell}=\widehat{M}$ samples, the sample variance is computed by the standard approach,

$$
\mathcal{V}\left(\Delta_{\ell} g ; M_{\ell}\right)=\frac{1}{M_{\ell}-1} \sum_{i=1}^{M_{\ell}}\left(\Delta_{\ell} g\left(\omega_{i, \ell}\right)-\mathcal{A}\left(\Delta_{\ell} g ; M_{\ell}\right)\right)^{2} .
$$

Thereafter, for every new batch of realizations, $\left\{\Delta_{\ell} g\left(\omega_{i, \ell}\right)\right\}_{i=M_{\ell}+1}^{M_{\ell}+M}(M$ here denotes an arbitrary natural number smaller or equal to 100,000), we incrementally update the sample variance,

$$
\begin{aligned}
\mathcal{V}\left(\Delta_{\ell} g ; M_{\ell}+M\right)= & \frac{M_{\ell}}{M_{\ell}+M} \times \mathcal{V}\left(\Delta_{\ell} g ; M_{\ell}\right) \\
& +\frac{1}{\left(M_{\ell}+M-1\right)} \sum_{i=M_{\ell}+1}^{M_{\ell}+M}\left(\Delta_{\ell} g\left(\omega_{i, \ell}\right)-\mathcal{A}\left(\Delta_{\ell} g ; M_{\ell}+M\right)\right)^{2},
\end{aligned}
$$

and update the total number of samples on level $\ell$ accordingly, $M_{\ell}=M_{\ell}+M$.

3.2.2. The Time Discretization Error. To control the time discretization error, we assume that a weak order convergence rate, $\alpha>0$, holds for the given SDE problem when solved with the Euler-Maruyama method, i.e.,

$$
\left|\mathrm{E}\left[g\left(X_{T}\right)-g\left(\bar{X}_{T}^{\{L\}}\right)\right]\right|=\mathcal{O}\left(N_{L}^{-\alpha}\right),
$$

and we assume that the asymptotic rate is reached at level $L-1$. Then

$$
\left|\mathrm{E}\left[g\left(X_{T}\right)-g\left(\bar{X}_{T}^{\{L\}}\right)\right]\right|=\left|\sum_{\ell=L+1}^{\infty} \mathrm{E}\left[\Delta_{\ell} g\right]\right| \leqslant\left|\mathrm{E}\left[\Delta_{L} g\right]\right| \sum_{\ell=1}^{\infty} 2^{-\alpha \ell}=\frac{\left|\mathrm{E}\left[\Delta_{L} g\right]\right|}{2^{\alpha}-1} .
$$

In our implementation, we assume the weak convergence rate, $\alpha$, is known prior to sampling and, replacing $\mathrm{E}\left[\Delta_{L} g\right]$ with a sample average approximation in the above inequality, we determine $L$ by the following stopping criterion:

$$
\frac{\max \left(2^{-\alpha}\left|\mathcal{A}\left(\Delta_{L-1} g ; M_{L-1}\right)\right|,\left|\mathcal{A}\left(\Delta_{L} g ; M_{L}\right)\right|\right)}{2^{\alpha}-1} \leqslant \mathrm{TOL}_{\mathrm{T}},
$$

(cf. Algorithm 3). Here we implicitly assume that the statistical error in estimating the bias condition is not prohibitively large.

A final level $L$ of order $\log \left(\mathrm{TOL}_{\mathrm{T}}{ }^{-1}\right)$ will thus control the discretization error.

3.2.3. Computational Cost. Under the convergence rate assumptions stated in Theorem 1.1 it follows that the cost of generating an adaptive MLMC estimator, $\mathcal{A}_{\mathcal{M L}}$, fulfilling the MSE approximation goal $\mathrm{E}\left[\left(\mathcal{A}_{\mathcal{M L}}-\mathrm{E}\left[g\left(X_{T}\right)\right]\right)^{2}\right] \leqslant \mathrm{TOL}^{2}$ is bounded by

$$
\mathcal{C}_{\mathcal{M L}}=\sum_{\ell=0}^{L} M_{\ell} C_{\ell} \leqslant \begin{cases}\mathcal{O}\left(\mathrm{TOL}^{-2}\right), & \text { if } \beta>1 \\ \mathcal{O}\left(\mathrm{TOL}^{-2} \log (\mathrm{TOL})^{4}\right), & \text { if } \beta=1 \\ \mathcal{O}\left(\mathrm{TOL}^{-2+\frac{\beta-1}{\alpha}} \log (\mathrm{TOL})^{2}\right), & \text { if } \beta<1\end{cases}
$$

Moreover, under the additional higher moment approximation rate assumption

$$
\mathrm{E}\left[\left|g\left(\bar{X}_{T}^{\{\ell\}}\right)-g\left(X_{T}\right)\right|^{2+\nu}\right]=\mathcal{O}\left(2^{-\beta+\nu / 2}\right),
$$


the complexity bound (47) also holds for fulfilling criterion (2) asymptotically as TOL $\downarrow 0,(\mathrm{cf}$. [5]).

3.3. MLMC Pseudocode. In this section, we present pseudocode for the implementation of the MSE adaptive MLMC algorithm. In addition to Algorithms 1 and 2, presented in Section 2.2.4, the implementation consists of Algorithms 3 and 4 . Algorithm 3 describes how the stopping criterion for the final level $L$ is implemented and how the multilevel estimator is generated, and Algorithm 4 describes the steps for generating a realization $\Delta_{\ell} g$.
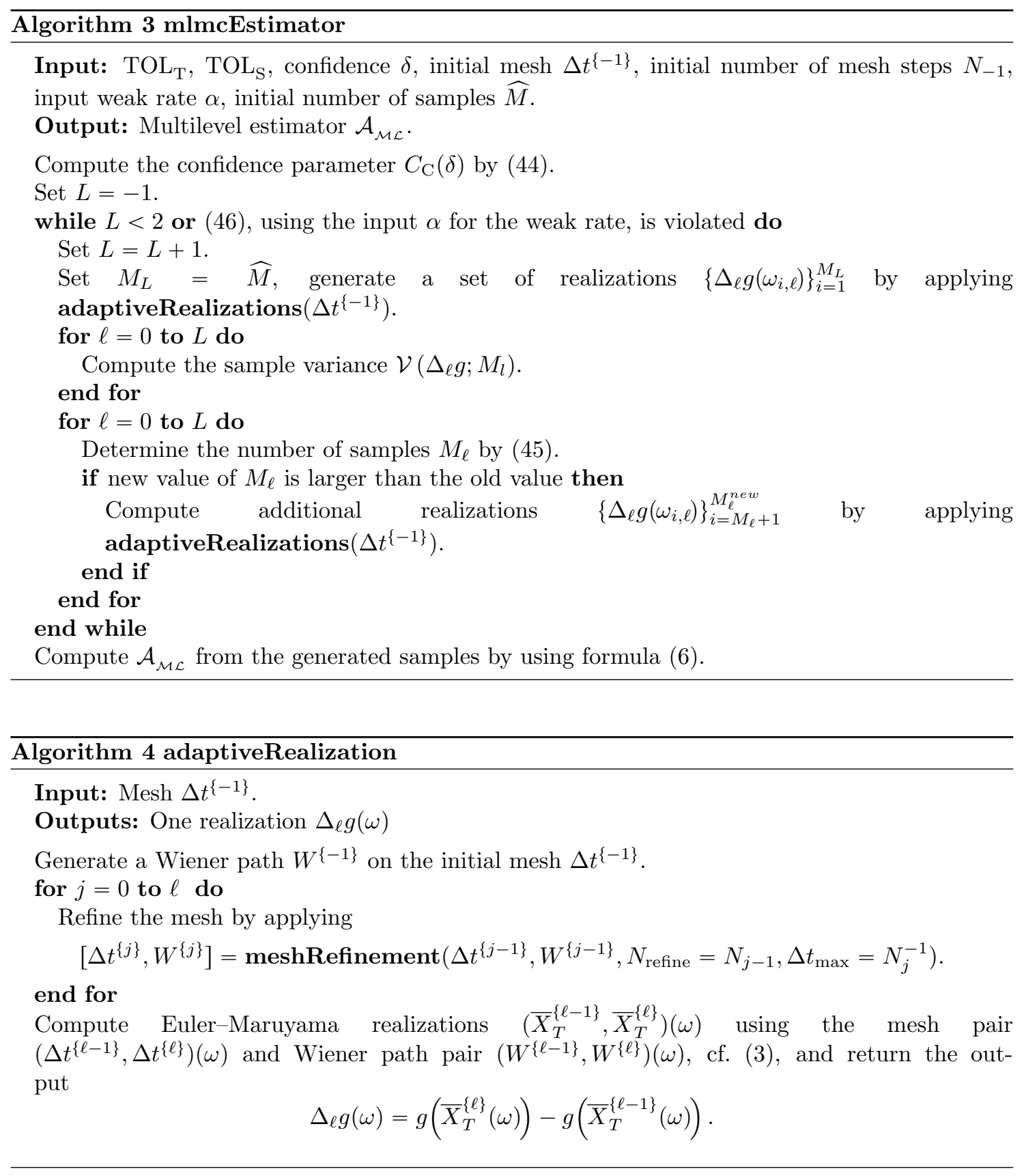

Remark 3.3. For each increment of $L$ in Algorithm 3 , all realizations $\Delta_{\ell} g$ that have been generated up to that point are reused in later computations of the multilevel estimator. This approach, which is common in MLMC, (cf. [8]), seems to work fine in practice although the independence between samples is then lost. Accounting for the lack of independence complicates the convergence analysis. 


\section{Numerical Examples for the MLmC Algorithms}

To illustrate the implementation of the MSE adaptive MLMC algorithm and to show its robustness and potential efficiency gain over the uniform MLMC algorithm, we present two numerical examples in this section. The first example considers a geometric Brownian motion SDE problem with sufficient regularity, such that there is very little (probably nothing) to gain by introducing adaptive mesh refinement. The example is included to show that in settings where adaptivity is not required, the MSE adaptive MLMC algorithm is not excessively more expensive than the uniform MLMC algorithm. In the second example, we consider an SDE with a random time drift coefficient blow-up of order $t^{-p}$ with $p \in[0.5,1)$. The MSE adaptive MLMC algorithm performs progressively more efficiently than does the uniform MLMC algorithm as the value of the blowup exponent $p$ increases. We should add, however, that although we observe numerical evidence for the numerical solutions converging for both examples, all of the assumptions in Theorem 2.1 are not fulfilled for our adaptive algorithm, when applied to either of the two examples. We are therefore not able to prove theoretically that our adaptive algorithm converges in these examples.

For reference, the implemented MSE adaptive MLMC algorithm is described in Algorithms 14 the standard form of the uniform time-stepping MLMC algorithm that we use in these numerical comparisons is presented in Algorithm 5. Appendix B, and a summary of the parameter values used in the examples is given in Table 2. Furthermore, all average properties derived from the MLMC algorithms that we plot for the considered examples in Figures 312 below are computed from 100 multilevel estimator realizations, and, when plotted, error bars are scaled to one sample standard deviation.

Example 4.1. We consider the geometric Brownian motion

$$
d X_{t}=X_{t} d t+X_{t} d W_{t}, \quad X_{0}=1
$$

where we seek to fulfill the weak approximation goal (2) for the observable, $g(x)=x$, at the final time, $T=1$. The reference solution is $\mathrm{E}\left[g\left(X_{T}\right)\right]=e^{T}$. From Example 2.1, we recall that the MSE minimized in this problem by using uniform time steps. However, our a posteriori MSE adaptive MLMC algorithm computes error indicators from numerical solutions of the path and the dual solution, which may lead to slightly non-uniform output meshes. In Figure 3, we study how close to uniform the MSE adaptive meshes are by plotting the level-wise ratio, $\mathrm{E}\left[\left|\Delta t^{\{\ell\}}\right|\right] / N_{\ell}$, where we recall that $\left|\Delta t^{\{\ell\}}\right|$ denotes the number of time steps in the mesh, $\Delta t^{\{\ell\}}$, and that a uniform mesh on level $\ell$ has $N_{\ell}$ time steps. As the level, $\ell$, increases, $\mathrm{E}\left[\left|\Delta t^{\{\ell\}}\right|\right] / N_{\ell}$ converges to 1 , and to interpret this result, we recall from the construction of the adaptive mesh hierarchy in Section 3 that if $\left|\Delta t^{\{\ell\}}\right|=N_{\ell}$, then the mesh, $\Delta t^{\{\ell\}}$, is uniform. We thus conclude that for this problem, the higher the level, the more uniform the MSE adaptive mesh realizations generally become.

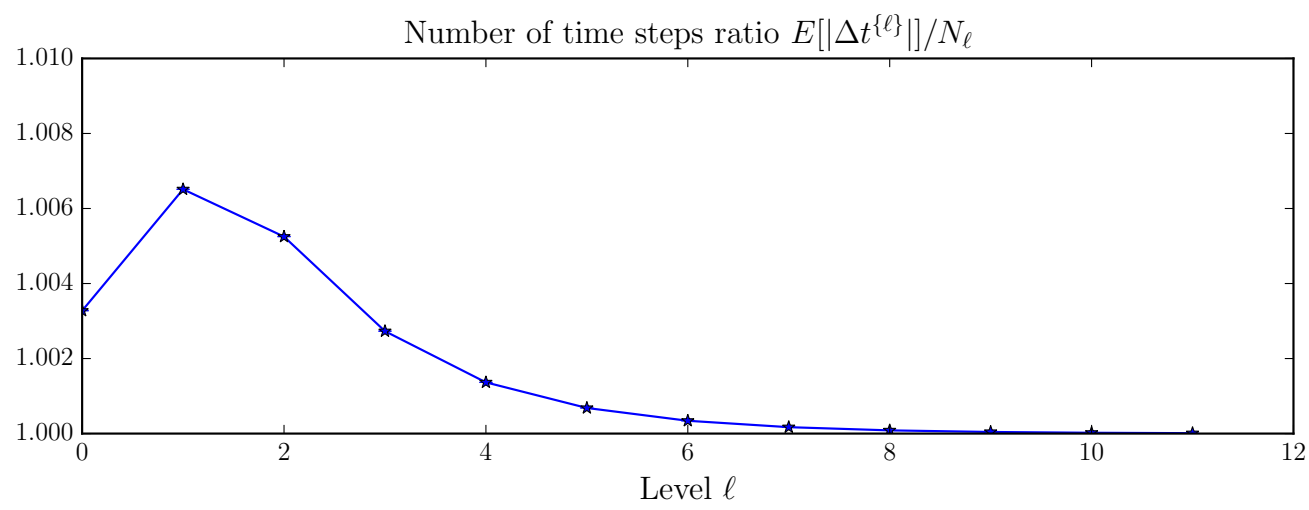

Figure 3. The ratio of the level-wise mean number of time steps $\mathrm{E}\left[\left|\Delta t^{\{\ell\}}\right|\right] / N_{\ell}$, of MSE adaptive mesh realizations to uniform mesh realizations for Example 4.2 . 
TABLE 2. List of parameter values used by the MSE adaptive MLMC algorithm and (when required) the uniform MLMC algorithm for the numerical examples in Section 4

\begin{tabular}{|c|c|c|c|}
\hline Parameter & Description of parameter & Example 4.1 & Example 4.2 \\
\hline$\delta$ & Confidence parameter, cf. $(39)$. & 0.1 & 0.1 \\
\hline TOL & Accuracy parameter, cf. (39). & {$\left[10^{-3}, 10^{-1}\right]$} & {$\left[10^{-3}, 10^{-1}\right]$} \\
\hline $\mathrm{TOL}_{\mathrm{S}}$ & $\begin{array}{l}\text { Statistical error tolerance, } \\
\text { cf. (40). }\end{array}$ & TOL $/ 2$ & $\mathrm{TOL} / 2$ \\
\hline $\mathrm{TOL}_{\mathrm{T}}$ & Bias error tolerance, cf. 400 & TOL $/ 2$ & TOL $/ 2$ \\
\hline$\Delta t^{\{-1\}}$ & $\begin{array}{l}\text { Pre-initial input uniform mesh } \\
\text { having the following step size. }\end{array}$ & $1 / 2$ & $1 / 2$ \\
\hline$N_{0}$ & $\begin{array}{l}\text { Number of time steps in the ini- } \\
\text { tial mesh } \Delta t^{\{0\}} \text {. }\end{array}$ & 4 & 4 \\
\hline$\tilde{N}(\ell)$ & $\begin{array}{l}\text { The number of complete updates } \\
\text { of the error indicators in the } \\
\text { MSE adaptive algorithm, cf. Al- } \\
\text { gorithm 1. }\end{array}$ & $\left.\frac{\log (\ell+2)}{\log (2)}\right\rfloor$ & $\left\lfloor\frac{\log (\ell+2)}{\log (2)}\right\rfloor$ \\
\hline$\Delta t_{\max }(\ell)$ & $\begin{array}{l}\text { Maximum permitted time step } \\
\text { size. }\end{array}$ & $N_{\ell}^{-1}$ & $N_{\ell}^{-1}$ \\
\hline$\Delta t_{\min }$ & $\begin{array}{l}\text { Minimum permitted time step } \\
\text { size (due to the used double- } \\
\text { precision binary floating-point } \\
\text { format). }\end{array}$ & $2^{-51}$ & $2^{-51}$ \\
\hline$\widehat{M}$ & $\begin{array}{l}\text { Number of first batch samples for } \\
\text { a (first) estimate of the variance } \\
\operatorname{Var}\left(\Delta_{\ell} g\right) \text {. }\end{array}$ & 100 & 20 \\
\hline$\alpha_{U}$ & $\begin{array}{l}\text { Input weak convergence rate } \\
\text { used in the stopping rule } 46 \\
\text { for uniform time step Euler- } \\
\text { Maruyama numerical integra- } \\
\text { tion. }\end{array}$ & 1 & $(1-p)$ \\
\hline$\alpha_{A}$ & $\begin{array}{l}\text { Input weak convergence rate } \\
\text { used in the stopping rule } 46 \\
\text { for the MSE adaptive time step } \\
\text { Euler-Maruyama numerical inte- } \\
\text { gration. }\end{array}$ & 1 & 1 \\
\hline
\end{tabular}

Since adaptive mesh refinement is costly and since this problem has sufficient regularity for the first-order weak and MSE convergence rates (4) and (5) to hold, respectively, one might expect that MSE adaptive MLMC will be less efficient than the uniform MLMC. This is verified in Figure 5, which shows that the runtime of the MSE adaptive MLMC algorithm grows slightly faster than the uniform MLMC algorithm and that the cost ratio is at most roughly 3.5, in favor of uniform MLMC. In Figure 4 the accuracy of the MLMC algorithms is compared, showing that both algorithms fulfill the goal (2) reliably. Figure 6 further shows that both algorithms have roughly first-order convergence rates for the weak error $\left|\mathrm{E}\left[\Delta_{\ell} g\right]\right|$ and the variance $\operatorname{Var}\left(\Delta_{\ell} g\right)$, and that the decay rates for $M_{l}$ are close to identical. We conclude that although MSE adaptive MLMC is slightly more costly than uniform MLMC, the algorithms perform comparably in terms of runtime for this example.

Remark 4.1. The reason why we are unable to prove theoretically that the numerical solution of this problem computed with our adaptive algorithm asymptotically converges to the true solution is slightly subtle. The required smoothness conditions in Theorem 2.1 are obviously fulfilled, but due 

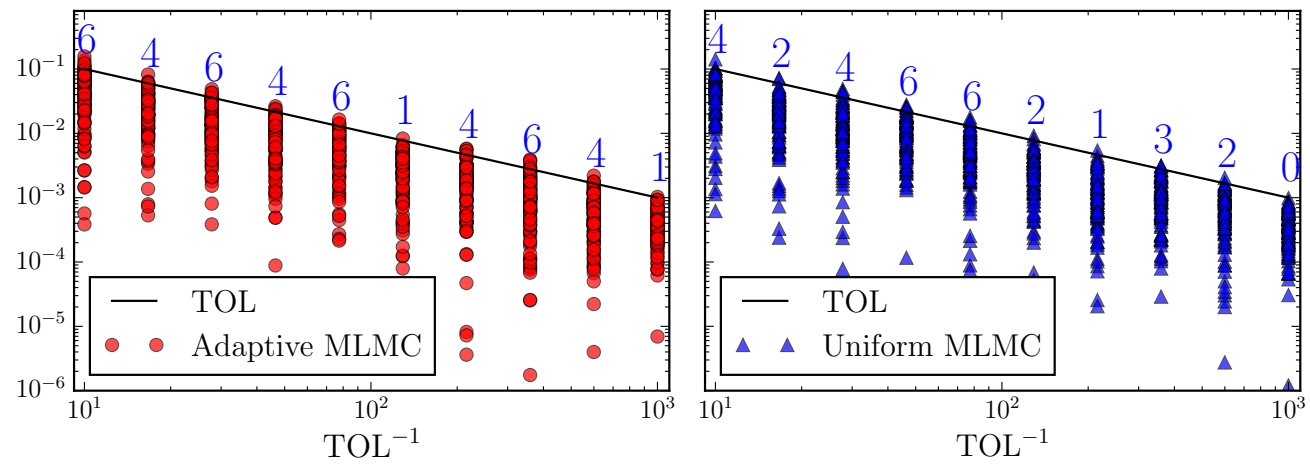

Figure 4. For a set of TOL values, 100 realizations of the MSE adaptive multilevel estimator are computed using both MLMC algorithms for Example 4.1. The errors $\mid \mathcal{A}_{\mathcal{M L}}\left(\omega_{i} ;\right.$ TOL,$\left.\delta\right)-\mathrm{E}\left[g\left(X_{T}\right)\right] \mid$ are respectively plotted as circles (adaptive MLMC) and triangles (uniform MLMC), and the number of multilevel estimator realizations failing the constraint $\left|\mathcal{A}_{\mathcal{M L}}\left(\omega_{i} ; \mathrm{TOL}, \delta\right)-\mathrm{E}\left[g\left(X_{T}\right)\right]\right|<$ TOL is written above the $\left(\mathrm{TOL}^{-1}, \mathrm{TOL}\right)$ line. Since the confidence parameter is set to $\delta=0.1$ and less than 10 realizations fail for any of the tested TOL values, both algorithms meet the approximation goal 339 .

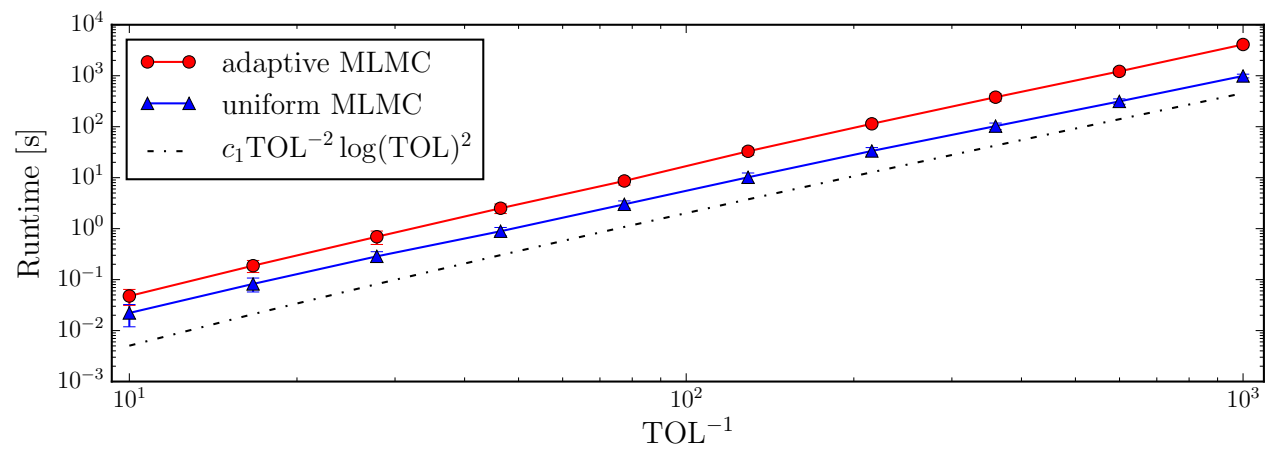

Figure 5. Average runtime vs. TOL ${ }^{-1}$ for the two MLMC algorithms solving Example 4.1.

to the local update of the error indicators in our mesh refinement procedure, (cf. Section 2.2.3), we cannot prove that the mesh points will asymptotically be stopping times for which $t_{n} \in \mathcal{F}_{t_{n-1}}$ for all $n \in\{1,2, \ldots, N\}$. If we instead were to use the version of our adaptive algorithm that recomputes all error indicators for each mesh refinement, the definition of the error density (26) implies that, for this particular problem, it would take the same value, $\bar{\rho}_{n}=\prod_{k=0}^{N-1} c_{x}\left(t_{k}, \bar{X}_{t_{k}}\right)^{2} / 2$, for all indices, $n \in\{0,1, \ldots, N\}$. The resulting adaptively refined mesh would then become uniform and we could verify convergence, for instance, by using Theorem 2.1. Connecting this to the numerical results for the adaptive algorithm that we have implemented here, we notice that the level-wise mean number of time steps ratio, $\mathrm{E}\left[\left|\Delta t^{\{\ell\}}\right|\right] / N_{\ell}$, presented in Figure 3 seems to tend towards 1 as $\ell$ increases, a limit ratio that is achieved only if $\Delta t^{\{\ell\}}$ is indeed a uniform mesh.

Example 4.2. We next consider the SDE

$$
\begin{aligned}
d X_{t} & =\underbrace{r f(t ; \xi) X_{t}}_{=: a\left(t, X_{t} ; \xi\right)} d t+\underbrace{\sigma X_{t}}_{=: b\left(t, X_{t} ; \xi\right)} d W_{t} \\
X_{0} & =1,
\end{aligned}
$$

with the low-regularity drift coefficient, $f(t ; \xi)=|t-\xi|^{-p}$, interest rate, $r=1 / 5$, volatility, $\sigma=0.5$, and observable, $g(x)=x$, at the final time $T=1$. A new singularity point, $\xi \in U(1 / 4,3 / 4)$, is 

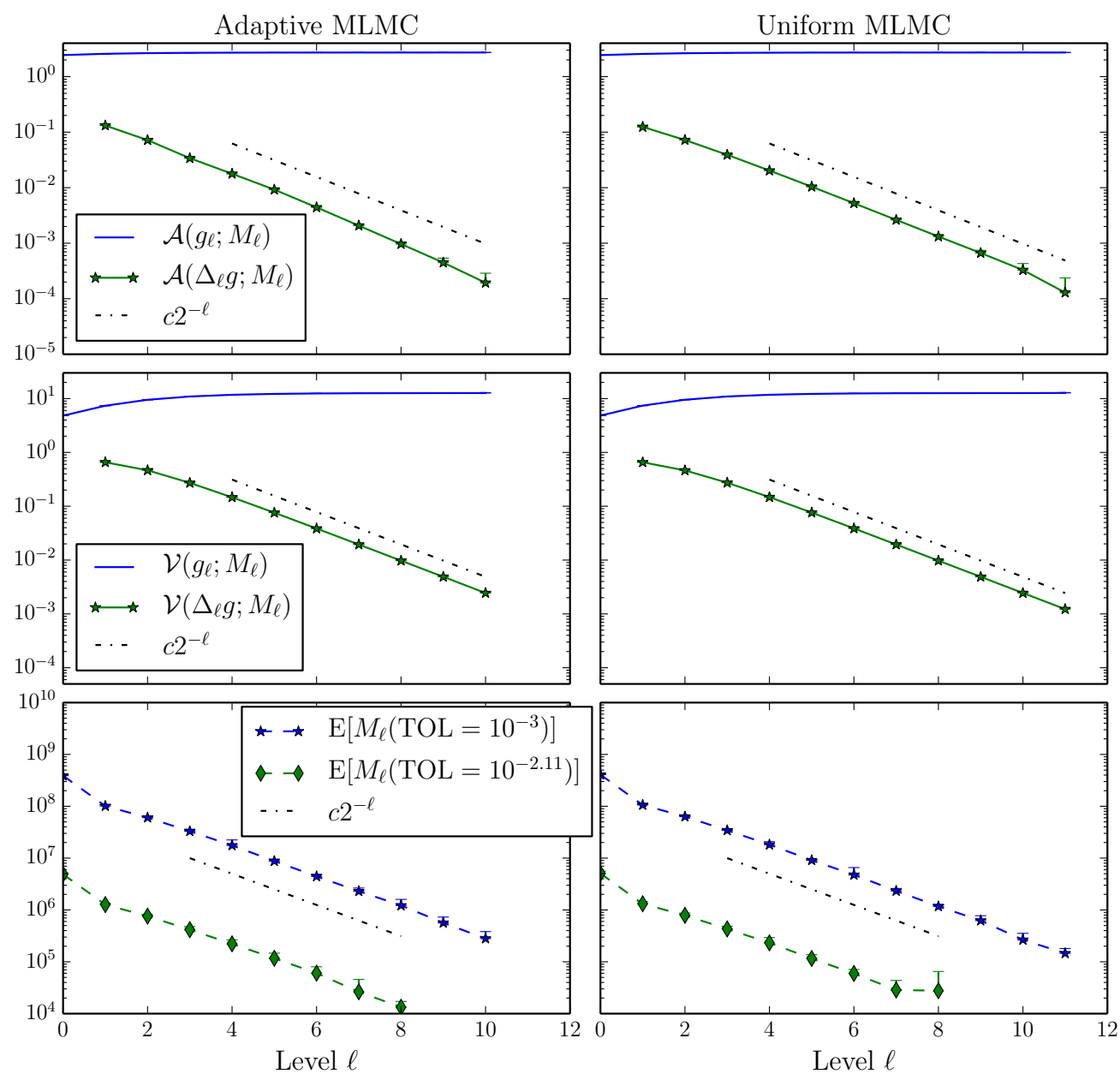

Figure 6. Output for Example 4.1 solved with the MSE adaptive and uniform time-stepping MLMC algorithms. (Top) Weak error $\left|\mathrm{E}\left[\Delta_{\ell} g\right]\right|$ for solutions at $\mathrm{TOL}=10^{-3}$. (Middle) Variance $\operatorname{Var}\left(\Delta_{\ell} g\right)$ for solutions at TOL $=10^{-3}$. (Bottom) Average number of samples $E\left[M_{l}\right]$.

sampled for each path, and it is independent from the Wiener paths, $W$. Three different blow-up exponent test cases are considered, $p=(1 / 2,2 / 3,3 / 4)$, and to avoid blow-ups in the numerical integration of the drift function component, $f(\cdot ; \xi)$, we replace the fully explicit Euler-Maruyama integration scheme with the following semi-implicit scheme:

$$
\bar{X}_{t_{n+1}}=\bar{X}_{t_{n}}+ \begin{cases}r f\left(t_{n} ; \xi\right) \bar{X}_{t_{n}} \Delta t_{n}+\sigma \bar{X}_{t_{n}} \Delta W_{n}, & \text { if } \quad f\left(t_{n} ; \xi\right)<2 f\left(t_{n+1} ; \xi\right), \\ r f\left(t_{n+1} ; \xi\right) \bar{X}_{t_{n}} \Delta t_{n}+\sigma \bar{X}_{t_{n}} \Delta W_{n}, & \text { else. }\end{cases}
$$

For $p \in[1 / 2,3 / 4]$ it may be shown that for any singularity point, any path integrated by the scheme (49) will have at most one drift-implicit integration step. The reference mean for the exact solution is given by

$$
\mathrm{E}\left[X_{T}\right]=2 \int_{1 / 4}^{3 / 4} \exp \left(\frac{r\left(x^{1-p}+(1-x)^{1-p}\right)}{1-p}\right) d x
$$

and in the numerical experiments, we approximate this integral value by quadrature to the needed accuracy.

The MSE Expansion for the Adaptive Algorithm. Due to the low-regularity drift present in this problem, the resulting MSE expansion will also contain drift-related terms that formally are of 
higher order. From the proof of Theorem 2.1, equation (61), we conclude that, to leading order the MSE is bounded by

$$
\mathrm{E}\left[\left|\bar{X}_{T}-X_{T}\right|^{2}\right] \leqslant \mathrm{E}\left[\sum_{n=0}^{N-1} \bar{\varphi}_{x, n}^{2} \frac{\left(N\left(a_{t}+a_{x} a\right)^{2} \Delta t_{n}^{2}+\left(b_{x} b\right)^{2}\right)\left(t_{n}, \bar{X}_{t_{n}} ; \xi\right)}{2} \Delta t_{n}^{2}\right] .
$$

This is the error expansion we use for the adaptive mesh refinement (in Algorithm 1) in this example. In Figure 7, we illustrate the effect that the singularity exponent, $p$, has on SDE and adaptive mesh realizations.
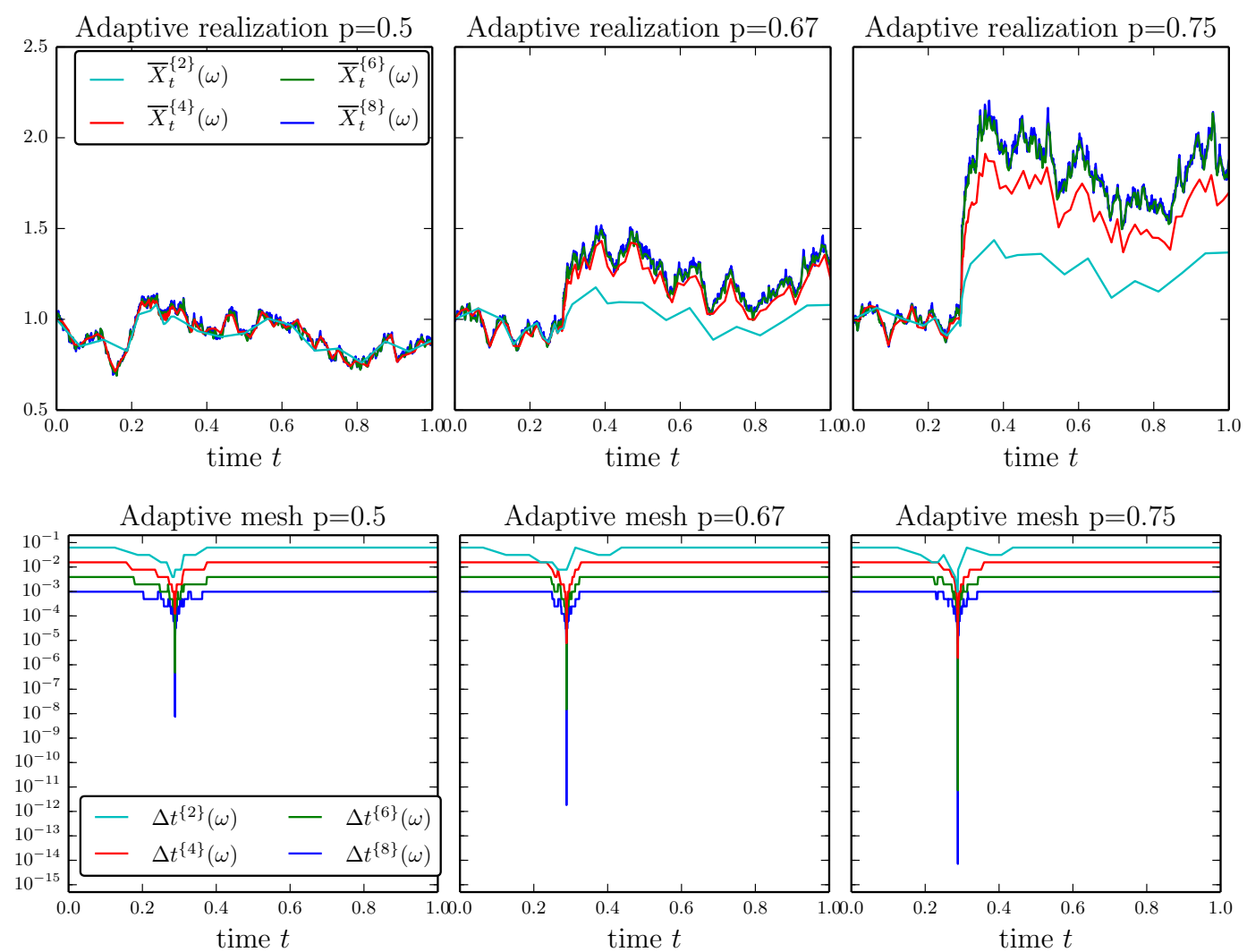

FigurE 7. (Top) One MSE adaptive numerical realization of the SDE problem (48) at different mesh hierarchy levels. The blow-up singularity point is located at $\xi \approx 0.288473$ and the realizations are computed for three singularity exponent values. We observe that as the exponent, $p$, increases, the more jump at $t=\xi$ becomes more pronounced. (Bottom) Corresponding MSE adaptive mesh realizations for the different test cases.

Implementation Details and Observations. Computational tests for the uniform and MSE adaptive MLMC algorithms are implemented with the input parameters summarized in Table 2. The weak convergence rate, $\alpha$, which is needed in the MLMC implementations' stopping criterion (46), is estimated experimentally as $\alpha(p)=(1-p)$ when using the Euler-Maruyama integrator with uniform time steps, and roughly $\alpha=1$ when using the Euler-Maruyama integrator with adaptive time steps, (cf. Figure 8). We further estimate the variance convergence rate to $\beta(p)=2(1-p)$, when using uniform time-stepping, and roughly to $\beta=1$ when using MSE adaptive time-stepping, (cf. Figure 9). The low weak convergence rate for uniform MLMC implies that the number of levels $L$ in the MLMC estimator will be become very large, even with fairly high tolerances. Since computations of realizations on high levels are extremely costly, we have, for the sake of 

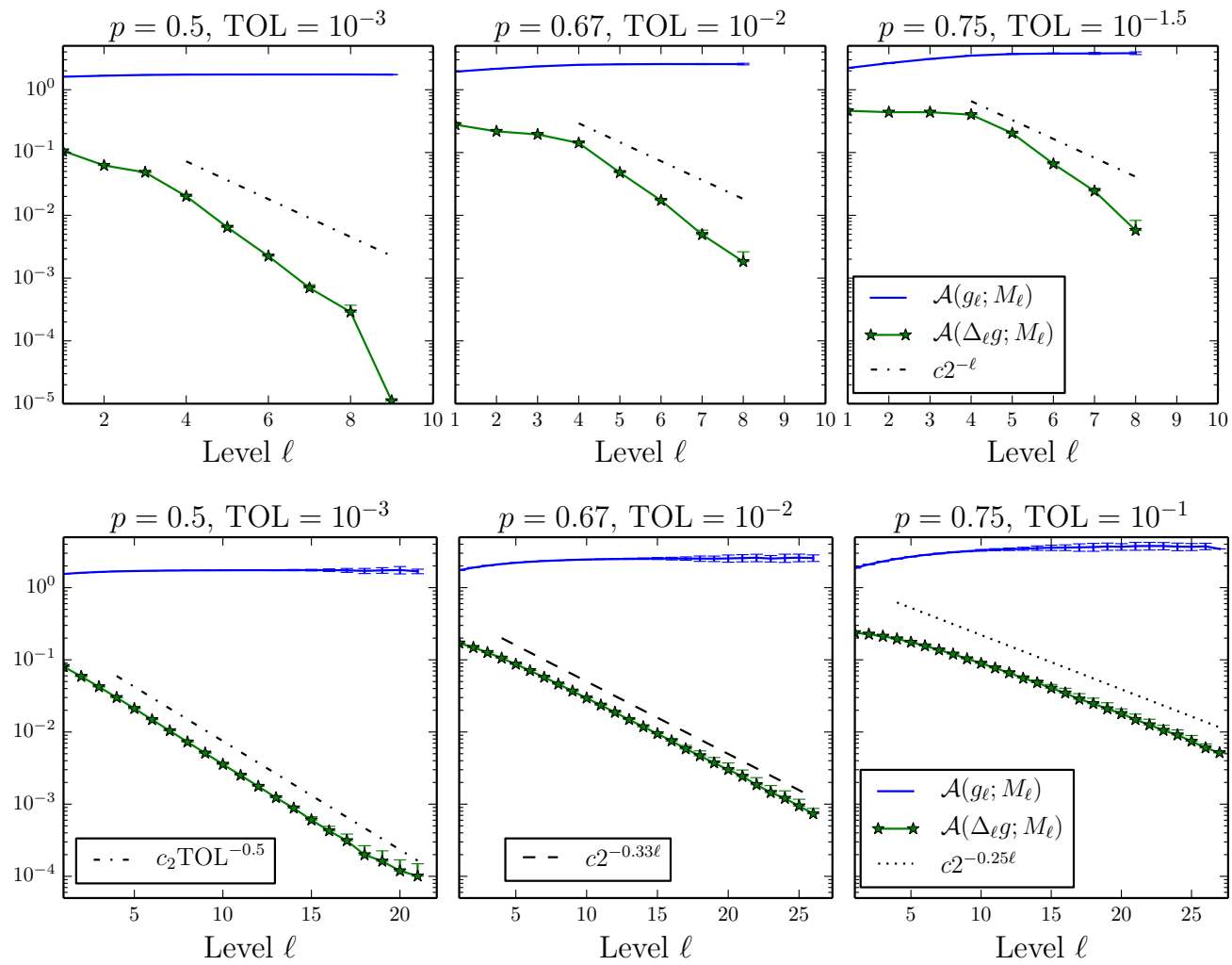

Figure 8. (Top) Average errors $\left|\mathrm{E}\left[\Delta_{\ell} g\right]\right|$ for Example 4.2 solved with the MSE adaptive MLMC algorithm for three singularity exponent values. (Bottom) Corresponding average errors for the uniform MLMC algorithm.

computational feasibility, chosen a very low value, $\widehat{M}=20$, for the initial number of samples in both MLMC algorithms. The respective estimators' use of samples, $M_{\ell}$, (cf. Figure 10), shows that the low number of initial samples is not strictly needed for the the adaptive MLMC algorithm, but for the sake of fair comparisons, we have chosen to use the same parameter values in both algorithms.

From the rate estimates of $\alpha$ and $\beta$, we predict the computational cost of reaching the approximation goal (39) for the respective MLMC algorithms to be

$$
\operatorname{Cost}_{\mathrm{adp}}\left(\mathcal{A}_{\mathcal{M L}}\right)=\mathcal{O}\left(\log (\mathrm{TOL})^{4} \mathrm{TOL}^{-2}\right) \quad \text { and } \quad \operatorname{Cost}_{\mathrm{unf}}\left(\mathcal{A}_{\mathcal{M L}}\right)=\mathcal{O}\left(\mathrm{TOL}^{-\frac{1}{1-p}}\right)
$$

by using the estimate 477 and Theorem 1.1 respectively. These predictions fit well with the observed computational runtime for the respective MLMC algorithms, (cf. Figure 11). Lastly, we observe that the numerical results are consistent with both algorithms fulfilling the goal $(39)$ in Figure 12 .

Computer Implementation. The computer code for all algorithms was written in Java and used the "Stochastic Simulation in Java" library to sample the random variables in parallel from thread-independent MRG32k3a pseudo random number generators, 24]. The experiments were run on multiple threads on Intel Xeon(R) CPU X5650, 2.67GHz processors and the computer graphics were made using the open source plotting library Matplotlib, [18.

\section{Conclusion}

We have developed an a posteriori, MSE adaptive Euler-Maruyama time-stepping algorithm and incorporated it into an MSE adaptive MLMC algorithm. The MSE error expansion presented 

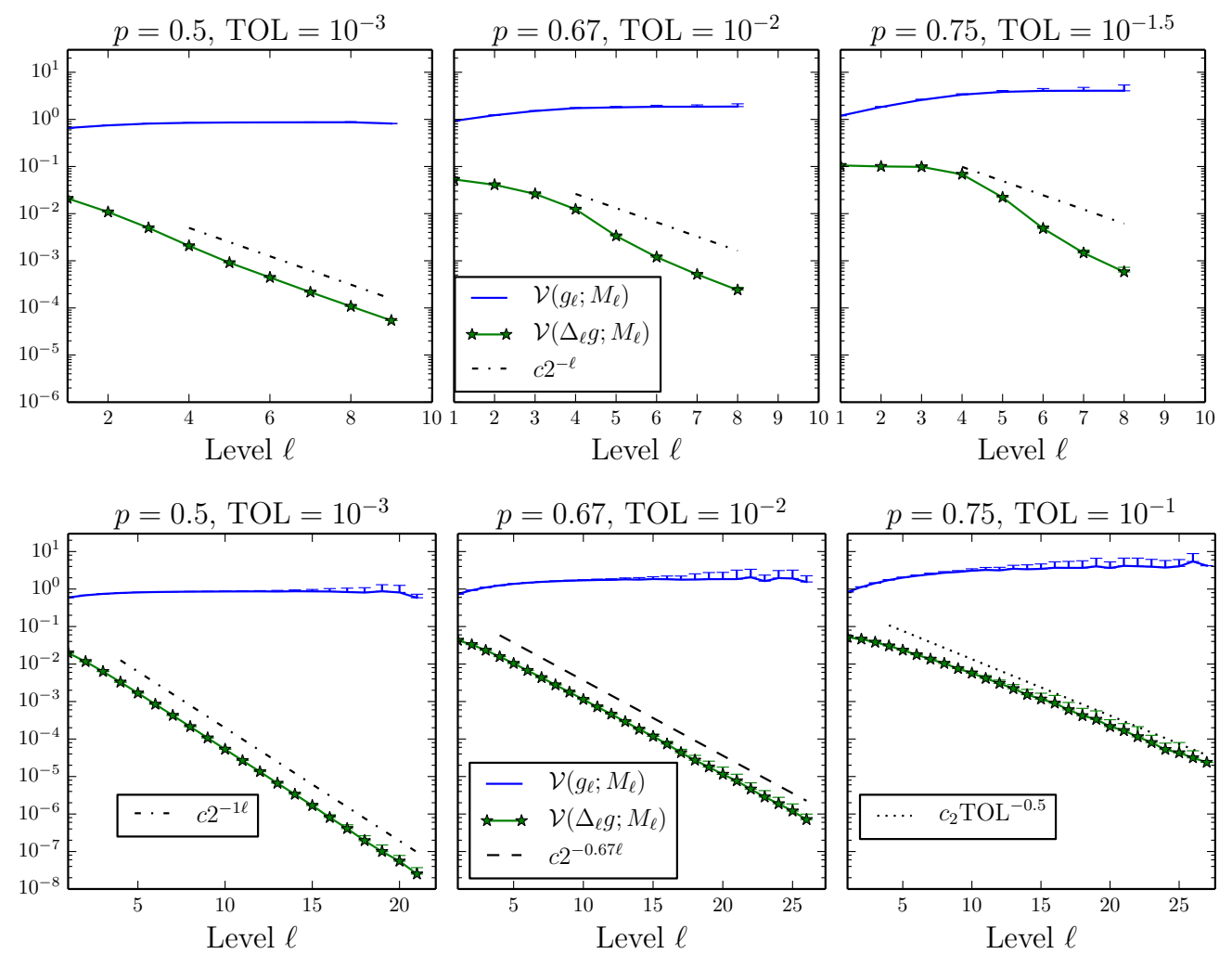

Figure 9. (Top) Variances $\operatorname{Var}\left(\Delta_{\ell} g\right)$ for for Example 4.2 solved with the MSE adaptive MLMC algorithm for three singularity exponent values. (Bottom) Corresponding variances for the uniform MLMC algorithm. The more noisy data on the highest levels is due to the low number used for the initial samples, $\hat{M}=20$, and only a subset of the generated 100 multilevel estimator realizations reached the last levels.

in Theorem 2.1 is fundamental to the adaptive algorithm. Numerical tests have shown that MSE adaptive time-stepping may outperform uniform time-stepping, both in the single-level MC setting and in the MLMC setting, (Examples 2.4 and 4.2). Due to the complexities of implementing adaptive time-stepping, the numerical examples in this work were restricted to quite simple, lowregularity SDE problems with singularities in the temporal coordinate. In the future, we aim to study SDE problems with low-regularity in the state coordinate (preliminary tests and analysis do however indicate that then some ad hoc molding of the adaptive algorithm is required).

Although a posteriori adaptivity has proven to be a very effective method for deterministic differential equations, the use of information from the future of the numerical solution of the dual problem makes it a somewhat unnatural method to extend to Itô SDE: It can result in numerical solutions that are not $\mathcal{F}_{t}$-adapted, which consequently may introduce a bias in the numerical solutions. [7 provides an example of a failing adaptive algorithm for SDE. A rigorous analysis of the convergence properties of our developed MSE adaptive algorithm would strengthen the theoretical basis of the algorithm further. We leave this for future work. 

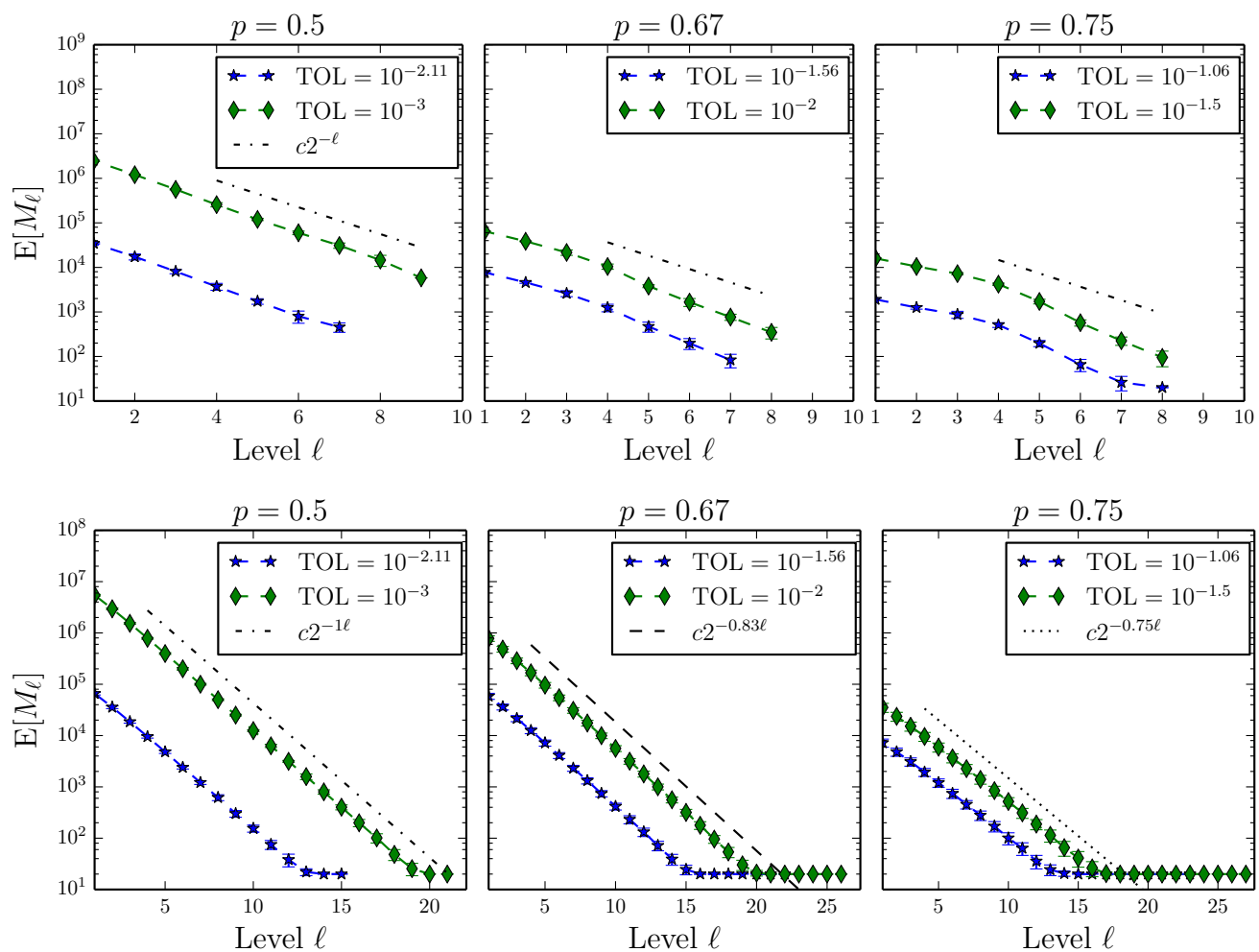

Figure 10. (Top) Average number of samples $M_{\ell}$ for for Example 4.2 solved with the MSE adaptive MLMC algorithm for three singularity exponent values. (Bottom) Corresponding average number of samples for the uniform MLMC algorithm. The plotted decay rate reference lines, $c 2^{-((\beta(p)+1) / 2) \ell}$, for $M_{\ell}$ follow implicitly from equation (45) (assuming that $\beta(p)=2(1-p)$ is the correct variance decay rate).
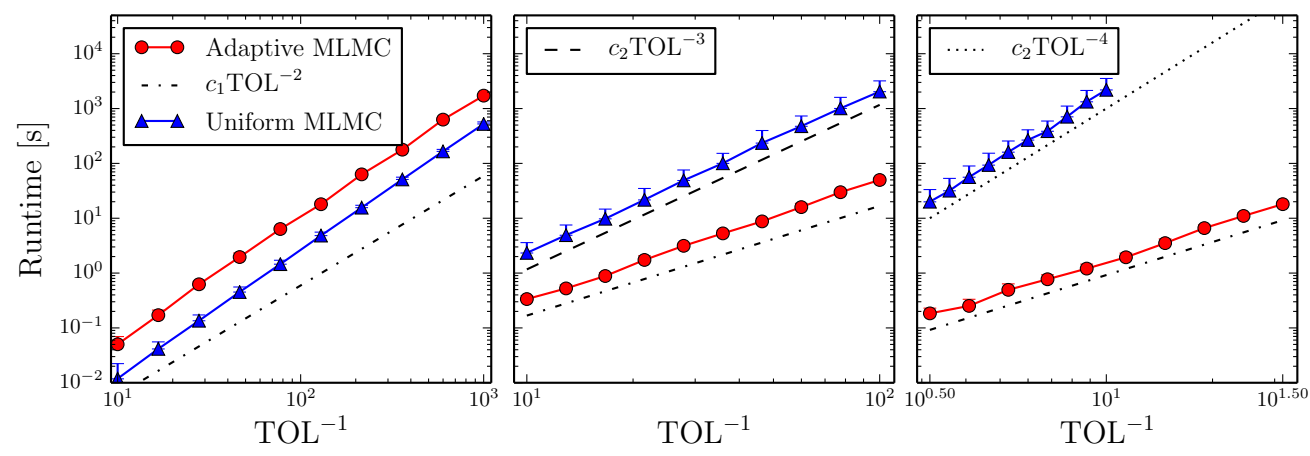

Figure 11. Average runtime vs. TOL ${ }^{-1}$ for the two MLMC algorithms for three singularity exponent values in Example 4.2 . 

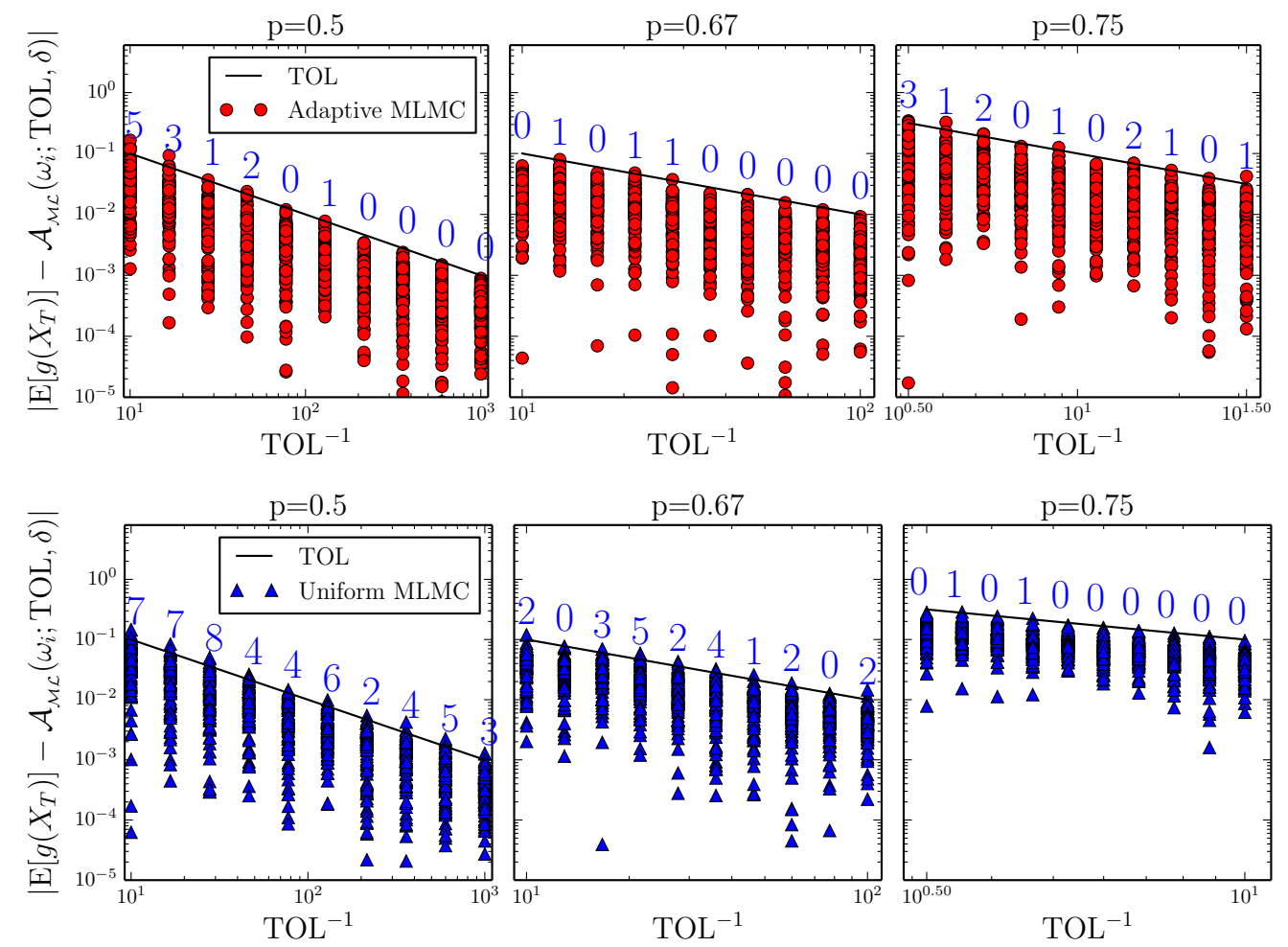

FiguRE 12. Approximation errors for both of the MLMC algorithms solving Example 4.2. At every TOL value, circles and triangles represent the errors from 100 independent multilevel estimator realizations of the respective algorithms.

\section{Appendix A. Theoretical Results}

A.1. Error Expansion for the MSE in 1D. In this section, we derive a leading-order error expansion for the MSE (11) in the 1D setting when the drift and diffusion coefficients are respectively mappings of the form $a:[0, T] \times \mathbb{R}$ and $b:[0, T] \times \mathbb{R} \rightarrow \mathbb{R}$. We begin by deriving a representation of the MSE in terms of products of local errors and weights.

Recalling the definition of the flow map, $\varphi(x, t):=g\left(X_{T}^{x, t}\right)$, and the first variation of the flow map and the path itself given in Section 2.1.1. we use the Mean Value Theorem to deduce that

$$
\begin{aligned}
g\left(X_{T}\right)-g\left(\bar{X}_{T}\right) & =\varphi\left(0, x_{0}\right)-\varphi\left(0, \bar{X}_{T}\right) \\
& =\sum_{n=0}^{N-1} \varphi\left(t_{n}, \bar{X}_{t_{n}}\right)-\varphi\left(t_{n+1}, \bar{X}_{t_{n+1}}\right) \\
& =\sum_{n=0}^{N-1} \varphi\left(t_{n+1}, X_{t_{n+1}}^{\bar{X}_{t_{n}}, t_{n}}\right)-\varphi\left(t_{n+1}, \bar{X}_{t_{n+1}}\right) \\
& =\sum_{n=0}^{N-1} \varphi_{x}\left(t_{n+1}, \bar{X}_{t_{n+1}}+s_{n} \Delta e_{n}\right) \Delta e_{n}
\end{aligned}
$$


where the local error is given by $\Delta e_{n}:=X_{t_{n+1}}^{\bar{X}_{t_{n}}, t_{n}}-\bar{X}_{t_{n+1}}$ and $s_{n} \in[0,1]$. Itô expansion of the local error gives the following representation:

$$
\begin{aligned}
& \Delta e_{n}=\underbrace{\int_{t_{n}}^{t_{n+1}} a\left(t, X_{t}^{X_{t_{n}}, t_{n}}\right)-a\left(t_{n}, \bar{X}_{t_{n}}\right) d t}_{\Delta a_{n}}+\underbrace{\int_{t_{n}}^{t_{n+1}} b\left(t, X_{t}^{\bar{X}_{t_{n}}, t_{n}}\right)-b\left(t_{n}, \bar{X}_{t_{n}}\right) d W_{t}}_{=: \widetilde{\Delta a_{n}}} \\
& =\underbrace{\int_{t_{n}}^{t_{n+1}} \int_{t_{n}}^{t}\left(a_{t}+a_{x} a+\frac{a_{x x}}{2} b^{2}\right)\left(s, X_{s}^{\bar{X}_{t_{n}}, t_{n}}\right) d s d t}_{\Delta b_{n}}+\underbrace{\int_{t_{n}}^{t_{n+1}} \int_{t_{n}}^{t}\left(a_{x} b\right)\left(s, X_{s}^{\bar{X}_{t_{n}}, t_{n}}\right) d W_{s} d t}_{=: \widetilde{\Delta b_{n}}} \\
& +\underbrace{\int_{t_{n}}^{t_{n+1}} \int_{t_{n}}^{t}\left(b_{t}+b_{x} a+\frac{b_{x x}}{2} b^{2}\right)\left(s, X_{s}^{\bar{X}_{t_{n}}, t_{n}}\right) d s d W_{t}}_{=: \widetilde{\Delta a_{n}}}+\underbrace{\int_{t_{n}}^{t_{n+1}} \int_{t_{n}}^{t}\left(b_{x} b\right)\left(s, X_{s}^{X_{t_{n}}, t_{n}}\right) d W_{s} d W_{t}}_{=: \widetilde{\Delta b_{n}}} .
\end{aligned}
$$

By equation (50) we may express the MSE by the following squared sum

$$
\begin{aligned}
& \mathrm{E}\left[\left(g\left(X_{T}\right)-g\left(\bar{X}_{T}\right)\right)^{2}\right]=\mathrm{E}\left[\left(\sum_{n=0}^{\check{N}-1} \varphi_{x}\left(t_{n+1}, \bar{X}_{t_{n+1}}+s_{n} \Delta e_{n}\right) \Delta e_{n}\right)^{2}\right] \\
& =\sum_{n, k=0}^{\check{N}-1} \mathrm{E}\left[\varphi_{x}\left(t_{k+1}, \bar{X}_{t_{k+1}}+s_{k} \Delta e_{k}\right) \varphi_{x}\left(t_{n+1}, \bar{X}_{t_{n+1}}+s_{n} \Delta e_{n}\right) \Delta e_{k} \Delta e_{n}\right] .
\end{aligned}
$$

This is the first step in deriving the error expansion in Theorem 2.1. The remaining steps follow in the proof below.

Proof of Theorem 2.1. The main tools used in proving this theorem are Taylor and Itô-Taylor expansions, Itô isometry, and truncation of higher order terms. For errors attributed to the leading-order local error term, $\widetilde{\Delta b} n$, (cf. equation (51)), we do detailed calculations, and the remainder is bounded by stated higher order terms.

We begin by noting that under the assumptions in Theorem 2.1 Lemmas 2.2 and 2.3 respectively verify then the existence and uniqueness of the solution of the SDE $X$ and the numerical solution $\bar{X}$, and provide higher order moment bounds for both. Furthermore, due to the assumption of the mesh points being stopping times for which $t_{n} \in \mathcal{F}_{t_{n-1}}$ for all $n$, it follows also that the numerical solution is adapted to the filtration, i.e., $\bar{X}_{t_{n}} \in \mathcal{F}_{t_{n}}$ for all $n$.

We further need to extend the flow map and the first variation notation from Section 2.1.1. Let $\bar{X}_{t_{n}}^{x, t_{k}}$ for $n \geqslant k$ denote the numerical solution of the Euler-Maruyama scheme

$$
\bar{X}_{t_{j+1}}^{x, t_{k}}=\bar{X}_{t_{j}}^{x, t_{k}}+a\left(t_{j}, \bar{X}_{t_{j}}^{x, t_{k}}\right) \Delta t_{j}+b\left(t_{j}, \bar{X}_{t_{j}}^{x, t_{k}}\right) \Delta W_{j}, \quad j \geqslant k,
$$

with initial condition $X_{t_{k}}=x$. The first variation of $\bar{X}_{t_{n}}^{x, t_{k}}$ is defined by $\partial_{x} \bar{X}_{t_{n}}^{x, t_{k}}$. Provided that $\mathrm{E}\left[|x|^{2 p}\right]<\infty$ for all $p \in \mathbb{N}, x \in \mathcal{F}_{t_{k}}$ and provided the assumptions of Lemma 2.3 hold, it is straightforward to extend the proof of the lemma to verify that $\left(\bar{X}^{x, t_{k}}, \partial_{x} \bar{X}^{x, t_{k}}\right)$ converges strongly to $\left(X^{x, t_{k}}, \partial_{x} X^{x, t_{k}}\right)$ for $t \in\left[t_{k}, T\right]$,

$$
\begin{array}{r}
\max _{k \leqslant n \leqslant N}\left(\left(\mathrm{E}\left[\left|\bar{X}_{t_{n}}^{x, t_{k}}-X_{t_{n}}^{x, t_{k}}\right|^{2 p}\right]\right)^{1 / 2 p}\right) \leqslant C \check{N}^{-1 / 2}, \quad \forall p \in \mathbb{N} \\
\max _{k \leqslant n \leqslant N}\left(\left(\mathrm{E}\left[\left|\partial_{x} \bar{X}_{t_{n}}^{x, t_{k}}-\partial_{x} X_{t_{n}}^{x, t_{k}}\right|^{2 p}\right]\right)^{1 / 2 p}\right) \leqslant C \check{N}^{-1 / 2}, \quad \forall p \in \mathbb{N}
\end{array}
$$

and

$$
\max _{k \leqslant n \leqslant N}\left(\max \left(\mathrm{E}\left[\left|\bar{X}_{t_{n}}^{x, t_{k}}\right|^{2 p}\right], \mathrm{E}\left[\left|\partial_{x} \bar{X}_{t_{n}}^{x, t_{k}}\right|^{2 p}\right]\right)\right)<\infty, \quad \forall p \in \mathbb{N} .
$$

In addition to this, we will also make use of moment bounds for the second and third variation of the flow map in the proof, i.e., $\varphi_{x x}(t, x)$ and $\varphi_{x x x}(t, x)$. The second variation is described in 
Section A.2. where it is shown in Lemma A.1 that provided that $x \in \mathcal{F}_{t}$ and $\mathrm{E}\left[|x|^{2 p}\right]<\infty$ for all $p \in \mathbb{N}$, then

$$
\max \left(\mathrm{E}\left[\left|\varphi_{x x}(t, x)\right|^{2 p}\right], \mathrm{E}\left[\left|\varphi_{x x x}(t, x)\right|^{2 p}\right], \mathrm{E}\left[\left|\varphi_{x x x x}(t, x)\right|^{2 p}\right]\right)<\infty, \quad \forall p \in \mathbb{N} .
$$

Considering the MSE error contribution from the leading order local error terms $\widetilde{\Delta b_{n}}$, i.e.,

$$
\mathrm{E}\left[\varphi_{x}\left(t_{k+1}, \bar{X}_{t_{k+1}}+s_{k} \Delta e_{k}\right) \varphi_{x}\left(t_{n+1}, \bar{X}_{t_{n+1}}+s_{n} \Delta e_{n}\right) \widetilde{\Delta b_{k}} \widetilde{\Delta b_{n}}\right]
$$

we have for $k=n$,

$$
\begin{aligned}
& \mathrm{E}\left[\left(\varphi_{x}\left(t_{n+1}, \bar{X}_{t_{n+1}}\right)+\varphi_{x x}\left(t_{n+1}, \bar{X}_{t_{n+1}}+\hat{s}_{n} \Delta e_{n}\right) s_{n} \Delta e_{n}\right)^{2} \widetilde{\Delta b}_{n}^{2}\right] \\
= & \mathrm{E}\left[\varphi_{x}\left(t_{n+1}, \bar{X}_{t_{n+1}}\right)^{2} \widetilde{\Delta b}_{n}^{2}+o\left(\Delta t_{n}^{2}\right)\right] .
\end{aligned}
$$

The above $o\left(\Delta t_{n}^{2}\right)$ follows from Young's and Hölder's inequalities,

$$
\begin{aligned}
& \mathrm{E}\left[2 \varphi_{x}\left(t_{n+1}, \bar{X}_{t_{n+1}}\right) \varphi_{x x}\left(t_{n+1}, \bar{X}_{t_{n+1}}+\hat{s}_{n} \Delta e_{n}\right) s_{n} \Delta e_{n} \widetilde{\Delta b}_{n}^{2}\right] \\
& \leqslant C\left(\mathrm{E}\left[\left(\varphi_{x}\left(t_{n+1}, \bar{X}_{t_{n+1}}\right) \varphi_{x x}\left(t_{n+1}, \bar{X}_{t_{n+1}}+\hat{s}_{n} \Delta e_{n}\right)\right)^{2} \Delta t_{n}^{3}\right]+\mathrm{E}\left[\frac{\Delta e_{n}^{2} \widetilde{\Delta b}_{n}^{4}}{\Delta t_{n}^{3}}\right]\right) \\
& \leqslant C\left(\mathrm{E}\left[\mathrm{E}\left[\left(\varphi_{x}\left(t_{n+1}, \bar{X}_{t_{n+1}}\right) \varphi_{x x}\left(t_{n+1}, \bar{X}_{n+1}+\hat{s}_{n} \Delta e_{n}\right)\right)^{2} \mid \mathcal{F}_{t_{n}}\right] \Delta t_{n}^{3}\right]\right. \\
& \left.+\mathrm{E}\left[\frac{\widetilde{\Delta a}_{n}^{2} \widetilde{\Delta b}_{n}^{4}}{\Delta t_{n}^{3}}\right]+\mathrm{E}\left[\frac{\widetilde{\Delta a}_{n}^{2} \widetilde{\Delta b}_{n}^{4}}{\Delta t_{n}^{3}}\right]+\mathrm{E}\left[\frac{\widetilde{\Delta b}_{n}^{2} \widetilde{\Delta b}_{n}^{4}}{\Delta t_{n}^{3}}\right]+\mathrm{E}\left[\frac{\widetilde{\Delta b}_{n}^{6}}{\Delta t_{n}^{3}}\right]\right) \\
& \leqslant C\left\{\mathrm{E}\left[\Delta t_{n}^{3}\right]+\left(\sqrt{\mathrm{E}\left[\mathrm{E}\left[\widetilde{\Delta a_{n}^{4}} \mid \mathcal{F}_{t_{n}}\right] \frac{1}{\Delta t_{n}}\right]}+\sqrt{\mathrm{E}\left[\mathrm{E}\left[\widetilde{\Delta a_{n}^{4}} \mid \mathcal{F}_{t_{n}}\right] \frac{1}{\Delta t_{n}}\right]}\right.\right. \\
& \left.\left.+\sqrt{\mathrm{E}\left[\mathrm{E}\left[\widetilde{\Delta b}_{n}^{4} \mid \mathcal{F}_{t_{n}}\right] \frac{1}{\Delta t_{n}}\right]}+\sqrt{\mathrm{E}\left[\mathrm{E}\left[\widetilde{\Delta b}_{n}^{4} \mid \mathcal{F}_{t_{n}}\right] \frac{1}{\Delta t_{n}}\right]}\right) \sqrt{\mathrm{E}\left[\mathrm{E}\left[\widetilde{\Delta b}_{n}^{8} \mid \mathcal{F}_{t_{n}}\right] \frac{1}{\Delta t_{n}^{5}}\right]}\right\} \\
& =\mathrm{E}\left[o\left(\Delta t_{n}^{2}\right)\right]
\end{aligned}
$$

where the last inequality is derived by applying the moment bounds for multiple Itô integrals described in [22, Lemma 5.7.5] and under the assumptions (R.1), (R.2), (M.1), (M.2) and (M.3). This yields

$$
\begin{aligned}
& \mathrm{E}\left[\widetilde{\Delta a}_{n}^{4} \mid \mathcal{F}_{t_{n}}\right] \leqslant C \mathrm{E}\left[\sup _{s \in\left[t_{n}, t_{n+1}\right)}\left|a_{t}+a_{x} a+\frac{a_{x x}}{2} b^{2}\right|^{4}\left(s, X_{s}^{\bar{X}_{t_{n}}, t_{n}}\right) \mid \mathcal{F}_{t_{n}}\right] \Delta t_{n}^{8}, \\
& \mathrm{E}\left[\widetilde{\Delta a_{n}^{4}} \mid \mathcal{F}_{t_{n}}\right] \leqslant C \mathrm{E}\left[\sup _{s \in\left[t_{n}, t_{n+1}\right)}\left|a_{x} b\right|^{4}\left(s, X_{s}^{\bar{X}_{t_{n}}, t_{n}}\right) \mid \mathcal{F}_{t_{n}}\right] \Delta t_{n}^{6}, \\
& \mathrm{E}\left[\widetilde{\Delta b}_{n}^{4} \mid \mathcal{F}_{t_{n}}\right] \leqslant C \mathrm{E}\left[\sup _{s \in\left[t_{n}, t_{n+1}\right)}\left|b_{t}+b_{x} a+\frac{b_{x x}}{2} b^{2}\right|^{4}\left(s, X_{s}^{\bar{X}_{t_{n}}, t_{n}}\right) \mid \mathcal{F}_{t_{n}}\right] \Delta t_{n}^{6} \text {, } \\
& \mathrm{E}\left[\widetilde{\Delta b}_{n}^{4} \mid \mathcal{F}_{t_{n}}\right] \leqslant C \mathrm{E}\left[\sup _{s \in\left[t_{n}, t_{n+1}\right)}\left|b_{x} b\right|^{4}\left(s, X_{s}^{\bar{X}_{t_{n}}, t_{n}}\right) \mid \mathcal{F}_{t_{n}}\right] \Delta t_{n}^{4}, \\
& \mathrm{E}\left[\widetilde{\Delta b}_{n}^{8} \mid \mathcal{F}_{t_{n}}\right] \leqslant C \mathrm{E}\left[\sup _{s \in\left[t_{n}, t_{n+1}\right)}\left|b_{x} b\right|^{8}\left(s, X_{s}^{\bar{X}_{t_{n}}, t_{n}}\right) \mid \mathcal{F}_{t_{n}}\right] \Delta t_{n}^{8} .
\end{aligned}
$$

And by similar reasoning,

$$
\mathrm{E}\left[\varphi_{x x}\left(\bar{X}_{t_{n+1}}+\hat{s}_{n} \Delta e_{n}, t_{n+1}\right)^{2} s_{n}^{2} \Delta e_{n}^{2} \widetilde{\Delta b}_{n}^{2}\right] \leqslant C \mathrm{E}\left[\Delta t_{n}^{4}\right]
$$


For achieving independence between forward paths and dual solutions in the expectations, an Itô-Taylor expansion of $\varphi_{x}$ leads to the equality

$$
\mathrm{E}\left[\varphi_{x}\left(t_{n+1}, \bar{X}_{t_{n+1}}\right)^{2} \widetilde{\Delta b}_{n}^{2}\right]=\mathrm{E}\left[\varphi_{x}\left(t_{n+1}, \bar{X}_{t_{n}}\right)^{2} \widetilde{\Delta b}_{n}^{2}+o\left(\Delta t_{n}^{2}\right)\right]
$$

Introducing the null set completed $\sigma$-algebra

$$
\widehat{\mathcal{F}}^{n}=\overline{\sigma\left(\sigma\left(\left\{W_{s}\right\}_{0 \leqslant s \leqslant t_{n}}\right) \vee \sigma\left(\left\{W_{s}-W_{t_{n+1}}\right\}_{t_{n+1} \leqslant s \leqslant T}\right)\right) \vee \sigma\left(X_{0}\right)},
$$

we observe that $\varphi_{x}\left(t_{n+1}, \bar{X}_{t_{n}}\right)^{2}$ is $\hat{\mathcal{F}}^{n}$ measurable by construction, (cf. [27, App. B]). Moreover, by conditional expectation,

$$
\begin{aligned}
\mathrm{E}\left[\varphi_{x}\left(t_{n+1}, \bar{X}_{t_{n}}\right)^{2} \widetilde{\Delta b}_{n}^{2}\right] & =\mathrm{E}\left[\varphi_{x}\left(t_{n+1}, \bar{X}_{t_{n}}\right)^{2} \mathrm{E}\left[\widetilde{\Delta b}_{n}^{2} \mid \widehat{\mathcal{F}}^{n}\right]\right] \\
& =\mathrm{E}\left[\varphi_{x}\left(t_{n+1}, \bar{X}_{t_{n}}\right)^{2}\left(b_{x} b\right)^{2}\left(t_{n}, \bar{X}_{t_{n}}\right) \frac{\Delta t_{n}^{2}}{2}+o\left(\Delta t_{n}^{2}\right)\right],
\end{aligned}
$$

where the last equality follows from using Itô's formula,

$$
\begin{aligned}
\left(b_{x} b\right)^{2}\left(t, X_{t}^{\bar{X}_{t_{n}}, t_{n}}\right)= & \left(b_{x} b\right)^{2}\left(t_{n}, \bar{X}_{t_{n}}\right)+\int_{t_{n}}^{t}\left(\left(\partial_{t}+a \partial_{x}+\frac{b^{2}}{2} \partial_{x}^{2}\right)\left(b_{x} b\right)^{2}\right)\left(s, X_{s}^{\bar{X}_{t_{n}}, t_{n}}\right) d s \\
& +\int_{t_{n}}^{t}\left(b \partial_{x}\left(b_{x} b\right)^{2}\right)\left(s, X_{s}^{\bar{X}_{t_{n}}, t_{n}}\right) d W_{s}, \quad t \in\left[t_{n}, t_{n+1}\right),
\end{aligned}
$$

to derive that

$$
\begin{aligned}
\mathrm{E}\left[\widetilde{\Delta b}_{n}^{2} \mid \widehat{\mathcal{F}}^{n}\right] & =\mathrm{E}\left[\left(\int_{t_{n}}^{t_{n+1}} \int_{t_{n}}^{t}\left(b_{x} b\right)\left(s, X_{s}^{\bar{X}_{t_{n}}, t_{n}}\right) d W_{s} d W_{t}\right)^{2} \mid \bar{X}_{t_{n}}\right] \\
& =\frac{\left(b_{x} b\right)^{2}\left(t_{n}, \bar{X}_{t_{n}}\right)}{2} \Delta t_{n}^{2}+o\left(\Delta t_{n}^{2}\right) .
\end{aligned}
$$

Here, the higher order $o\left(\Delta t_{n}^{2}\right)$ terms are bounded in a similar fashion as the terms in inequality (55), by using [22, Lemma 5.7.5].

For the terms in (54) for which $k<n$, we will show that

$$
\sum_{k, n=0}^{\check{N}-1} \mathrm{E}\left[\varphi_{x}\left(t_{k+1}, \bar{X}_{t_{k+1}}+s_{k} \Delta e_{k}\right) \varphi_{x}\left(t_{n+1}, \bar{X}_{t_{n+1}}+s_{n} \Delta e_{n}\right) \widetilde{\Delta b_{k}} \widetilde{\Delta b_{n}}\right]=\sum_{n=0}^{\check{N}-1} \mathrm{E}\left[o\left(\Delta t_{n}^{2}\right)\right],
$$

which means that the contribution to the MSE from these terms is negligible to leading order. For the use in later expansions, let us first observe by use of the chain rule that for any $y \in \mathcal{F}_{t_{n}}$ with bounded second moment,

$$
\begin{aligned}
\varphi_{x}\left(t_{k+1}, y\right) & =g^{\prime}\left(X_{T}^{y, t_{k+1}}\right) \partial_{x} X_{T}^{y, t_{k+1}} \\
& =g^{\prime}\left(X_{T}^{\bar{X}_{t_{k+1}}+s_{m} \Delta e_{k}, t_{k+1}}\right) \partial_{x} X_{T}^{X_{t_{n+1}}^{y, t_{k+1}, t_{n+1}} \partial_{x} X_{t_{n+1}}^{y, t_{k+1}}} \\
& =\varphi_{x}\left(t_{n+1}, X_{t_{n+1}}^{y, t_{k+1}}\right) \partial_{x} X_{t_{n+1}}^{y, t_{k+1}}
\end{aligned}
$$

and that

$$
\begin{aligned}
\partial_{x} X_{t_{n+1}}^{\bar{X}_{t_{k+1}}+s_{k} \Delta e_{k}, t_{k+1}} & =\partial_{x} X_{t_{n}}^{\bar{X}_{t_{k+1}}+s_{k} \Delta e_{k}, t_{k+1}} \\
+ & \int_{t_{n}}^{t_{n+1}} a_{x}\left(s, X_{s}^{\bar{X}_{t_{k+1}}+s_{k} \Delta e_{k}, t_{k+1}}\right) \partial_{x} X_{s}^{\bar{X}_{t_{k+1}}+s_{k} \Delta e_{k}, t_{k+1}} d s \\
& \quad+\int_{t_{n}}^{t_{n+1}} b_{x}\left(s, X_{s}^{\bar{X}_{t_{k+1}}+s_{k} \Delta e_{k}, t_{k+1}}\right) \partial_{x} X_{s}^{X_{t_{k+1}}+s_{k} \Delta e_{k}, t_{k+1}} d W_{s} .
\end{aligned}
$$

We next introduce the $\sigma$-algebra

$$
\widehat{\mathcal{F}}^{k, n}:=\overline{\sigma\left(\left\{W_{s}\right\}_{0 \leqslant s \leqslant t_{k}}\right) \vee \sigma\left(\left\{W_{s}-W_{t_{k+1}}\right\}_{t_{k+1} \leqslant s \leqslant t_{n}}\right) \vee \sigma\left(\left\{W_{s}-W_{t_{n+1}}\right\}_{t_{n+1} \leqslant s \leqslant T}\right) \vee \sigma\left(X_{0}\right)},
$$


and Itô-Taylor expand the $\varphi_{x}$ functions in (57) about center points that are $\widehat{\mathcal{F}}^{k, n}$-measurable:

$$
\begin{aligned}
& \varphi_{x}\left(t_{k+1}, \bar{X}_{t_{k+1}}+s_{k} \Delta e_{k}\right)=\varphi_{x}\left(t_{n+1}, X_{t_{n+1}}^{\bar{X}_{t_{k+1}}+s_{k} \Delta e_{k}, t_{k+1}}\right) \partial_{x} X_{t_{n+1}}^{\bar{X}_{t_{k+1}}+s_{k} \Delta e_{k}, t_{k+1}} \\
& =\left[\varphi_{x}\left(t_{n+1}, X_{t_{n}}^{\bar{X}_{t_{k}}, t_{k+1}}\right)+\varphi_{x x}\left(t_{n+1}, X_{t_{n}}^{\bar{X}_{t_{k}}, t_{k+1}}\right)\left(X_{t_{n+1}}^{\bar{X}_{t_{k+1}}+s_{k} \Delta e_{k}, t_{k+1}}-X_{t_{n}}^{\bar{X}_{t_{k}}, t_{k+1}}\right)\right. \\
& +\varphi_{x x x}\left(t_{n+1}, X_{t_{n}}^{\bar{X}_{t_{k}}, t_{k+1}}\right) \frac{\left(X_{t_{n+1}}^{\bar{X}_{t_{k+1}}+s_{k} \Delta e_{k}, t_{k+1}}-X_{t_{n}}^{\bar{X}_{t_{k}}, t_{k+1}}\right)^{2}}{2} \\
& +\varphi_{x x x x}\left(t_{n+1},\left(1-\check{s}_{n}\right) X_{t_{n}}^{\bar{X}_{t_{k}}, t_{k+1}}+\check{s}_{n} X_{t_{n+1}}^{\bar{X}_{t_{k+1}}+s_{k} \Delta e_{k}, t_{k+1}}\right)
\end{aligned}
$$

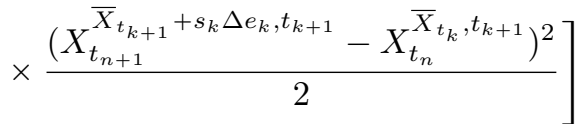

$$
\begin{aligned}
& \times\left[\partial_{x} X_{t_{n}}^{\bar{X}_{t_{k}}, t_{k+1}}+\partial_{x x} X_{t_{n}}^{\bar{X}_{t_{k}}, t_{k+1}}\left(a\left(t_{k}, \bar{X}_{t_{k}}\right) \Delta t_{k}+b\left(t_{k}, \bar{X}_{t_{k}}\right) \Delta W_{k}+s_{k} \Delta e_{k}\right)\right. \\
& +\partial_{x x x} X_{t_{n}}^{\bar{X}_{t_{k}}+\grave{s}_{k}\left(a\left(t_{k}, \bar{X}_{t_{k}}\right) \Delta t_{k}+\left(b\left(t_{k}, \bar{X}_{t_{k}}\right) \Delta W_{k}+s_{k} \Delta e_{k}\right), t_{k+1}\right.} \\
& \times \frac{\left(a\left(t_{k}, \bar{X}_{t_{k}}\right) \Delta t_{k}+b\left(t_{k}, \bar{X}_{t_{k}}\right) \Delta W_{k}+s_{k} \Delta e_{k}\right)^{2}}{2} \\
& +\int_{t_{n}}^{t_{n+1}} a_{x}\left(s, X_{s}^{\bar{X}_{t_{k+1}}+s_{k} \Delta e_{k}, t_{k+1}}\right) \partial_{x} X_{s}^{\bar{X}_{t_{k+1}}+s_{k} \Delta e_{k}, t_{k+1}} d s \\
& \left.+\int_{t_{n}}^{t_{n+1}} b_{x}\left(s, X_{s}^{\bar{X}_{t_{k+1}}+s_{k} \Delta e_{k}, t_{k+1}}\right) \partial_{x} X_{s}^{\bar{X}_{t_{k+1}}+s_{k} \Delta e_{k}, t_{k+1}} d W_{s}\right],
\end{aligned}
$$

where

$$
\begin{aligned}
& X_{t_{n+1}}^{\bar{X}_{t_{k+1}}+s_{k} \Delta e_{k}, t_{k+1}}-X_{t_{n}}^{\bar{X}_{t_{k}}, t_{k+1}} \\
& =\int_{t_{n}}^{t_{n+1}} a\left(s, X_{s}^{\bar{X}_{t_{k+1}}+s_{k} \Delta e_{k}, t_{k+1}}\right) d s+\int_{t_{n}}^{t_{n+1}} b\left(s, X_{s}^{\bar{X}_{t_{k+1}}+s_{k} \Delta e_{k}, t_{k+1}}\right) d W_{s} \\
& +\partial_{x} X_{t_{n}}^{\bar{X}_{t_{k}}+\tilde{s}_{k}\left(a\left(t_{k}, \bar{X}_{t_{k}}\right) \Delta t_{k}+b\left(t_{k}, \bar{X}_{t_{k}}\right) \Delta W_{k}+s_{k} \Delta e_{k}\right), t_{k+1}}\left(a\left(t_{k}, \bar{X}_{t_{k}}\right) \Delta t_{k}+b\left(t_{k}, \bar{X}_{t_{k}}\right) \Delta W_{k}+s_{k} \Delta e_{k}\right),
\end{aligned}
$$

and

$$
\begin{aligned}
\varphi_{x}\left(t_{n+1}, \bar{X}_{t_{n+1}}+s_{n} \Delta e_{n}\right) & =\varphi_{x}\left(t_{n+1}, \bar{X}_{t_{n}} \bar{X}_{t_{k}}, t_{k+1}\right) \\
& +\varphi_{x x}\left(t_{n+1}, \bar{X}_{t_{n}}^{\bar{X}_{t_{k}}, t_{k+1}}\right) \Delta \nu_{k, n}+\varphi_{x x x}\left(t_{n+1}, \bar{X}_{n}^{X_{k}, t_{k+1}}\right) \frac{\Delta \nu_{k, n}^{2}}{2} \\
& +\varphi_{x x x x}\left(t_{n+1},\left(1-\dot{s}_{n}\right) \bar{X}_{t_{n}}^{X_{t_{k}}, t_{k+1}}+\dot{s}_{n}\left(\bar{X}_{t_{n+1}}+s_{n} \Delta e_{n}\right)\right) \frac{\Delta \nu_{k, n}^{3}}{6}
\end{aligned}
$$

with

$$
\begin{aligned}
\Delta \nu_{k, n} & :=a\left(t_{n}, \bar{X}_{t_{n}}\right) \Delta t_{n}+b\left(t_{n}, \bar{X}_{t_{n}}\right) \Delta W_{n}+s_{n} \Delta e_{n} \\
& +\partial_{x} \bar{X}_{t_{n}}^{\bar{X}_{t_{k}}+\hat{s}_{k}\left(a\left(t_{k}, \bar{X}_{t_{k}}\right) \Delta t_{k}+b\left(t_{k}, \bar{X}_{t_{k}}\right) \Delta W_{k}\right), t_{k+1}}\left(a\left(t_{k}, \bar{X}_{t_{k}}\right) \Delta t_{k}+b\left(t_{k}, \bar{X}_{t_{k}}\right) \Delta W_{k}+s_{k} \Delta e_{k}\right) .
\end{aligned}
$$

Plugging the expansions 58 and (59) into the expectation

$$
\mathrm{E}\left[\varphi_{x}\left(t_{k+1}, \bar{X}_{k+1}+s_{k} \Delta e_{k}\right) \varphi_{x}\left(t_{n+1}, \bar{X}_{n+1}+s_{n} \Delta e_{n}\right) \widetilde{\Delta b_{k}} \widetilde{\Delta b_{n}}\right]
$$


the summands in the resulting expression that only contains products of the first variations vanishes,

$$
\begin{aligned}
& \mathrm{E}\left[\varphi_{x}\left(t_{n+1}, X_{t_{n}}^{\bar{X}_{t_{k}}, t_{k+1}}\right) \partial_{x} X_{t_{n}}^{\bar{X}_{t_{k}}, t_{k+1}} \varphi_{x}\left(t_{n+1}, \bar{X}_{t_{n}}^{\bar{X}_{t_{k+1}}, t_{k+1}}\right) \widetilde{\Delta b_{k}} \widetilde{\Delta b_{n}}\right] \\
& =\mathrm{E}\left[\mathrm{E}\left[\widetilde{\Delta b_{n}} \widetilde{\Delta b}_{k} \mid \hat{\mathcal{F}}^{k, n}\right] \varphi_{x}\left(t_{n+1}, X_{t_{n}}^{\bar{X}_{t_{k}}, t_{k+1}}\right) \partial_{x} X_{t_{n}}^{\bar{X}_{t_{k}}, t_{k+1}} \varphi_{x}\left(t_{n+1}, \bar{X}_{t_{n}}^{\bar{X}_{t_{k}}, t_{k+1}}\right)\right]=0 .
\end{aligned}
$$

One can further deduce that all of the the summands in which the product of multiple Itô integrals $\widetilde{\Delta b_{k}}$ and $\widetilde{\Delta b_{n}}$ are multiplied only with one additional Itô integral of first-order vanish by using the fact that the inner product of the resulting multiple Itô integrals is zero, cf. [22, Lemma 5.7.2], and by separating the first and second variations from the Itô integrals by taking a conditional expectation with respect to the suitable filtration. We illustrate this with a couple of examples,

$$
\begin{aligned}
& \mathrm{E}\left[\varphi_{x}\left(t_{n+1}, X_{t_{n}}^{\bar{X}_{t_{k}}, t_{k+1}}\right) \partial_{x x} X_{t_{n}}^{\bar{X}_{t_{k}}, t_{k+1}} b\left(t_{k}, \bar{X}_{t_{k}}\right) \Delta W_{k} \varphi_{x}\left(t_{n+1}, \bar{X}_{t_{n}}^{\bar{X}_{t_{k}}, t_{k+1}}\right) \widetilde{\Delta b_{k}} \widetilde{\Delta b_{n}}\right] \\
& =\mathrm{E}\left[\varphi_{x}\left(t_{n+1}, X_{t_{n}}^{\bar{X}_{t_{k}}, t_{k+1}}\right) \partial_{x x} X_{t_{n}}^{\bar{X}_{t_{k}}, t_{k+1}} b\left(t_{k}, \bar{X}_{t_{k}}\right) \Delta W_{k} \varphi_{x}\left(t_{n+1}, \bar{X}_{t_{n}}^{\bar{X}_{t_{k}}, t_{k+1}}\right) \widetilde{\Delta b_{k}}\right. \\
& \left.\times \mathrm{E}\left[\widetilde{\Delta b_{n}} \mid \hat{\mathcal{F}}^{n}\right]\right]=0
\end{aligned}
$$

and

$$
\begin{aligned}
& \mathrm{E}\left[\varphi_{x}\left(t_{n+1}, X_{t_{n}}^{\bar{X}_{t_{k}}, t_{k+1}}\right) \partial_{x} X_{t_{n}}^{\bar{X}_{t_{k}}, t_{k+1}} b\left(t_{n}, \bar{X}_{t_{n}}\right) \Delta W_{n} \varphi_{x}\left(t_{n+1}, \bar{X}_{t_{n}}^{\bar{X}_{t_{k}}, t_{k+1}}\right) \widetilde{\Delta b} \widetilde{\Delta b}_{n}\right] \\
& =\mathrm{E}\left[\varphi_{x}\left(t_{n+1}, X_{t_{n+1}}^{\bar{X}_{t_{k}}, t_{k+1}}\right) \varphi_{x}\left(t_{n+1}, \bar{X}_{t_{n}}^{\bar{X}_{t_{k}}, t_{k+1}}\right) \widetilde{\Delta b_{k}} b\left(t_{n}, \bar{X}_{t_{n}}\right) \mathrm{E}\left[\widetilde{\Delta b_{n}} \Delta W_{n} \mid \hat{\mathcal{F}}^{n}\right]\right]=0 .
\end{aligned}
$$

From these observations, assumption (M.3), inequality (56), and, when necessary, additional expansions of integrands to render the leading order integrand either $\hat{\mathcal{F}}^{k}$ - or $\hat{\mathcal{F}}^{n}$-measurable and thereby sharpen the bounds (an example of such an expansion is

$$
\begin{aligned}
\widetilde{\Delta b_{n}} & =\int_{t_{n}}^{t_{n+1}} \int_{t_{n}}^{t}\left(b_{x} b\right)\left(s, X_{s}^{\bar{X}_{t_{n}}, t_{n}}\right) d W_{s} d W_{t} \\
& \left.=\int_{t_{n}}^{t_{n+1}} \int_{t_{n}}^{t}\left(b_{x} b\right)\left(s, X_{s}^{\bar{X}_{t_{n}}^{\bar{X}_{t_{k}}, t_{k+1}}, t_{n}}\right) d W_{s} d W_{t}+\text { h.o.t. }\right) .
\end{aligned}
$$

We derive after a laborious computation which we will not include here that

$$
\left|\mathrm{E}\left[\varphi_{x}\left(t_{k+1}, \bar{X}_{t_{k+1}}+s_{k} \Delta e_{k}\right) \varphi_{x}\left(t_{n+1}, \bar{X}_{t_{n+1}}+s_{n} \Delta e_{n}\right) \widetilde{\Delta b_{k}} \widetilde{\Delta b_{n}}\right]\right| \leqslant C \check{N}^{-3 / 2} \sqrt{\mathrm{E}\left[\Delta t_{k}^{2}\right] \mathrm{E}\left[\Delta t_{n}^{2}\right]} .
$$

This further implies that

$$
\begin{aligned}
& \sum_{k, n=0, k \neq n}^{\check{N}-1} \mathrm{E}\left[\varphi_{x}\left(t_{k+1}, \bar{X}_{t_{k+1}}+s_{k} \Delta e_{k}\right) \varphi_{x}\left(t_{n+1}, \bar{X}_{t_{n+1}}+s_{n} \Delta e_{n}\right) \widetilde{\Delta b_{k}} \widetilde{\Delta b}_{n}\right] \\
& \leqslant C \check{N}^{-3 / 2} \sum_{k, n=0, k \neq n}^{\check{N}-1} \sqrt{\mathrm{E}\left[\Delta t_{k}^{2}\right] \mathrm{E}\left[\Delta t_{n}^{2}\right]} \\
& \leqslant C \check{N}^{-3 / 2}\left(\sum_{n=0}^{\check{N}-1} \sqrt{\mathrm{E}\left[\Delta t_{n}^{2}\right]}\right)^{2} \\
& \leqslant C \check{N}^{-1 / 2} \sum_{n=0}^{\check{N}-1} \mathrm{E}\left[\Delta t_{n}^{2}\right]
\end{aligned}
$$


such that inequality (57) holds.

So far, we have shown that

$$
\begin{aligned}
\mathrm{E}\left[\left(\sum_{n=0}^{N-1} \varphi_{x}\left(t_{n+1}, \bar{X}_{t_{n+1}}+s_{n} \Delta e_{n}\right) \widetilde{\Delta b_{n}}\right)^{2}\right] \\
=\mathrm{E}\left[\sum_{n=0}^{N-1} \varphi_{x}\left(t_{n+1}, \bar{X}_{t_{n}}\right)^{2} \frac{\left(b_{x} b\right)^{2}}{2}\left(t_{n}, \bar{X}_{t_{n}}\right) \Delta t_{n}^{2}+o\left(\Delta t_{n}^{2}\right)\right] .
\end{aligned}
$$

The MSE contribution from the other local error terms, $\widetilde{\Delta a_{n}}, \widetilde{\Delta a_{n}}$ and $\widetilde{\Delta b}$, can also be bounded using the above approach with Itô-Taylor expansions, $\widehat{\mathcal{F}}^{m, n}$-conditioning and Itô isometries. This yields that

$$
\begin{gathered}
\mathrm{E}\left[\varphi_{x}\left(t_{k+1}, \bar{X}_{t_{k+1}}+s_{k} \Delta e_{k}\right) \varphi_{x}\left(t_{n+1}, \bar{X}_{t_{n+1}}+s_{n} \Delta e_{n}\right) \widetilde{\Delta a} \widetilde{\Delta a}_{k}\right] \\
=\mathrm{E}\left[\varphi_{x}\left(\bar{X}_{t_{k}}, t_{k}\right) \varphi_{x}\left(t_{n}, \bar{X}_{t_{n}}\right)\left(\frac{a_{t}+a_{x} a+a_{x x} b^{2} / 2}{2}\right)\left(t_{k}, \bar{X}_{t_{k}}\right) \times\right. \\
\left.\mathrm{E}\left[\frac{a_{t}+a_{x} a+a_{x x} b^{2} / 2}{2}\right)\left(t_{n}, \bar{X}_{t_{n}}\right) \Delta t_{k}^{2} \Delta t_{n}^{2}+o\left(\Delta t_{k}^{2} \Delta t_{n}^{2}\right)\right], \\
= \begin{cases}\left.\mathrm{E}\left[\bar{X}_{t_{k+1}}+t_{n}, \bar{X}_{t_{n}}\right)^{2} \frac{\left(a_{x} b\right)^{2}}{2}\left(t_{n}, \bar{X}_{t_{n}}\right) \Delta t_{n}^{3}+o\left(\Delta t_{n}^{3}\right)\right], & \text { if } k=n, \\
\mathcal{O}\left(\check{N}^{-3 / 2}\left(\mathrm{E}\left[\Delta t_{k}^{3}\right] \mathrm{E}\left[\Delta t_{n}^{3}\right]\right)^{1 / 2}\right), & \text { if } k \neq n,\end{cases}
\end{gathered}
$$

and

$$
\begin{aligned}
& \mathrm{E}\left[\varphi_{x}\left(t_{k+1}, \bar{X}_{t_{k+1}}+s_{k} \Delta e_{k}\right) \varphi_{x}\left(t_{n+1}, \bar{X}_{t_{n+1}}+s_{n} \Delta e_{n}\right) \widetilde{\Delta b}_{k} \widetilde{\Delta b}_{n}\right] \\
& \quad= \begin{cases}\mathrm{E}\left[\varphi_{x}\left(t_{n}, \bar{X}_{t_{n}}\right)^{2} \frac{\left(b_{t}+b_{x} a+b_{x x} b^{2} / 2\right)^{2}}{3}\left(t_{n}, \bar{X}_{t_{n}}\right) \Delta t_{n}^{3}+o\left(\Delta t_{n}^{3}\right)\right], & \text { if } k=n, \\
\mathcal{O}\left(\check{N}^{-3 / 2}\left(\mathrm{E}\left[\Delta t_{k}^{3}\right] \mathrm{E}\left[\Delta t_{n}^{3}\right]\right)^{1 / 2}\right), & \text { if } k \neq n .\end{cases}
\end{aligned}
$$

Moreover, conservative bounds for error contributions involving products of different local error terms, e.g., $\widetilde{\Delta a} \widetilde{\Delta b}_{n}$, can be induced from the above bounds and Hölder's inequality. For example,

$$
\begin{aligned}
& \left|\mathrm{E}\left[\sum_{k, n=0}^{\check{N}-1} \varphi_{x}\left(t_{k+1}, \bar{X}_{t_{k+1}}+s_{k} \Delta e_{k}\right) \widetilde{\Delta a} a_{k} \varphi_{x}\left(t_{n+1}, \bar{X}_{t_{n+1}}+s_{n} \Delta e_{n}\right) \widetilde{\Delta b_{n}}\right]\right| \\
& =\left|\mathrm{E}\left[\left(\sum_{k=0}^{\check{N}-1} \varphi_{x}\left(t_{k+1}, \bar{X}_{t_{k+1}}+s_{k} \Delta e_{k}\right) \widetilde{\Delta a_{k}}\right)\left(\sum_{k=0}^{\check{N}-1} \varphi_{x}\left(t_{n+1}, \bar{X}_{t_{n+1}}+s_{n} \Delta e_{n}\right) \widetilde{\Delta b_{n}}\right)\right]\right| \\
& \leqslant \sqrt{\left.\mathrm{E}\left[\left(\sum_{k=0}^{\check{N}-1} \varphi_{x}\left(t_{k+1}, \bar{X}_{t_{k+1}}+s_{k} \Delta e_{k}\right) \widetilde{\Delta a}\right)_{k}\right)^{2}\right] \mathrm{E}\left[\left(\sum_{n=0}^{\check{N}-1} \varphi_{x}\left(t_{n+1}, \bar{X}_{t_{n+1}}+s_{n} \Delta e_{n}\right) \widetilde{\Delta b_{n}}\right)^{2}\right]} \\
& =\mathcal{O}\left(\check{N}^{-1 / 2} \sum_{n=0}^{\check{N}-1} \mathrm{E}\left[\Delta t_{n}^{2}\right]\right) .
\end{aligned}
$$

The proof is completed in two replacement steps applied to $\varphi_{x}$ on the right-hand side of equality (60). First, we replace $\varphi_{x}\left(t_{n+1}, \bar{X}_{t_{n}}\right)$ by $\varphi_{x}\left(t_{n}, \bar{X}_{t_{n}}\right)$. Under the regularity assumed in this 
theorem, the replacement is possible without introducing additional leading order error terms as

$$
\begin{aligned}
& \left.\mathrm{E}\left[\left|\varphi_{x}\left(t_{n+1}, \bar{X}_{t_{n}}\right)-\varphi_{x}\left(t_{n}, \bar{X}_{t_{n}}\right)\right|\right]=\mathrm{E}\left[\left|g^{\prime}\left(X_{T}^{\bar{X}_{t_{n}}, t_{n+1}}\right) \partial_{x} X_{T}^{\bar{X}_{t_{n}}, t_{n+1}}-g^{\prime}\left(X_{T}^{\bar{X}_{t_{n}}, t_{n}}\right) \partial_{x} X_{T}^{\bar{X}_{t_{n}}, t_{n}}\right|\right]\right] \\
& \left.\leqslant \mathrm{E}\left[\left|\left(g^{\prime}\left(X_{T}^{\bar{X}_{t_{n}}, t_{n+1}}\right)-g^{\prime}\left(X_{T}^{\bar{X}_{t_{n}}, t_{n}}\right)\right) \partial_{x} X_{T}^{\bar{X}_{t_{n}}, t_{n+1}}\right|\right]\right] \\
& \quad+\mathrm{E}\left[\left|g^{\prime}\left(X_{T}^{\bar{X}_{t_{n}}, t_{n}}\right)\left(\partial_{x} X_{T}^{\bar{X}_{t_{n}}, t_{n+1}}-\partial_{x} X_{T}^{\bar{X}_{t_{n}}, t_{n}}\right)\right|\right] \\
& \quad \mathcal{O}\left(\check{N}^{-1 / 2}\right) .
\end{aligned}
$$

Here, the last equality follows from the assumptions (M.2), (M.3), (R.2), and (R.3), and Lemmas 2.2 and 2.3 .

$$
\begin{aligned}
& \mathrm{E}\left[\left|\left(g^{\prime}\left(X_{T}^{\bar{X}_{t_{n}}, t_{n+1}}\right)-g^{\prime}\left(X_{T}^{\bar{X}_{t_{n}}, t_{n}}\right)\right) \partial_{x} X_{T}^{\bar{X}_{t_{n}}, t_{n+1}}\right|\right]
\end{aligned}
$$

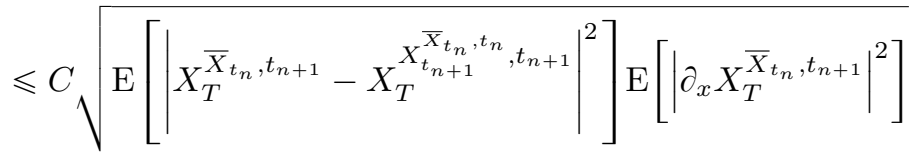

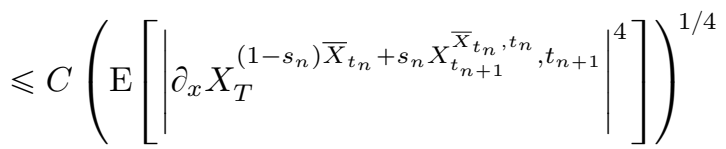

$$
\begin{aligned}
& \times\left(\mathrm{E}\left[\left|\int_{t_{n}}^{t_{n+1}} a\left(s, X_{s}^{\bar{X}_{t_{n}}, t_{n}}\right) d s+\int_{t_{n}}^{t_{n+1}} b\left(s, X_{s}^{\bar{X}_{t_{n}}, t_{n}}\right) d W_{s}\right|^{4}\right]\right)^{1 / 4} \\
& \leqslant C\left(\mathrm{E}\left[\sup _{t_{n} \leqslant s \leqslant t_{n+1}}\left|a\left(s, X_{s}^{\bar{X}_{t_{n}}, t_{n}}\right)\right|^{4} \Delta t_{n}^{4}+\sup _{t_{n} \leqslant s \leqslant t_{n+1}}\left|b\left(s, X_{s}^{\bar{X}_{t_{n}}, t_{n}}\right)\right|^{4} \Delta t_{n}^{2}\right]\right)^{1 / 4} \\
& =\mathcal{O}\left(\check{N}^{-1 / 2}\right) \text {, }
\end{aligned}
$$

and that

$$
\begin{aligned}
& \mathrm{E}\left[\left|g^{\prime}\left(X_{T}^{\bar{X}_{t_{n}}, t_{n}}\right)\left(\partial_{x} X_{T}^{\bar{X}_{t_{n}}, t_{n+1}}-\partial_{x} X_{T}^{\bar{X}_{t_{n}}, t_{n}}\right)\right|\right] \leqslant C \sqrt{\mathrm{E}\left[\left|\partial_{x} X_{T}^{\bar{X}_{t_{n}}, t_{n+1}}-\partial_{x} X_{T}^{\bar{X}_{t_{n}}, t_{n}}\right|^{2}\right]} \\
& =C \sqrt{\mathrm{E}\left[\mid \partial_{x} X_{T}^{\bar{X}_{t_{n}}, t_{n+1}}-\partial_{x} X_{T}^{X_{t_{n+1}}^{\bar{X}_{t_{n}, t_{n}}, t_{n+1}}} \partial_{x} X_{t_{n+1}}^{\left.\left.\bar{X}_{t_{n}, t_{n}}\right|^{2}\right]}\right.} \\
& \leqslant C\left(\sqrt{\mathrm{E}\left[\mid \partial_{x} X_{T}^{\bar{X}_{t_{n}}, t_{n+1}}-\partial_{x} X_{T}^{\left.X_{t_{n+1}}^{\bar{X}_{t_{n}, t_{n}}, t_{n+1}} \mid\right]}\right.}\right.
\end{aligned}
$$

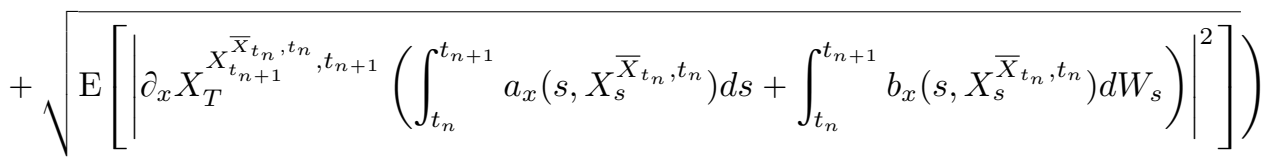

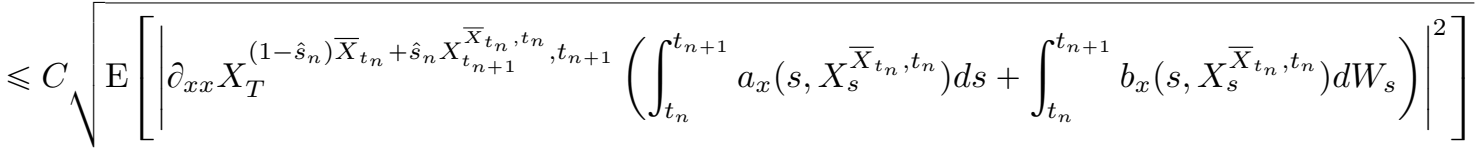

$$
\begin{aligned}
& +\mathcal{O}\left(\check{N}^{-1 / 2}\right) \\
& =\mathcal{O}\left(\check{N}^{-1 / 2}\right) \text {. }
\end{aligned}
$$

The last step is to replace the first variation of the exact path $\varphi_{x}\left(t_{n}, \bar{X}_{t_{n}}\right)$ with the first variation of the numerical solution $\bar{\varphi}_{x, n}=g^{\prime}\left(\bar{X}_{T}\right) \partial_{x} \bar{X}_{T}^{\bar{X}_{t_{n}}, t_{n}}$. This is also possible without introducing 
additional leading order error terms by the same assumptions and similar bounding arguments as in the two preceding bounds as

$$
\begin{aligned}
& \mathrm{E}\left[\left|\bar{\varphi}_{x, n}-\varphi_{x}\left(t_{n}, \bar{X}_{t_{n}}\right)\right|\right]=\mathrm{E}\left[\left|g^{\prime}\left(\bar{X}_{T}\right) \partial_{x} \bar{X}_{T}^{\bar{X}_{t_{n}}, t_{n}}-g^{\prime}\left(X_{T}^{\bar{X}_{t_{n}}, t_{n}}\right) \partial_{x} X_{T}^{\bar{X}_{t_{n}}, t_{n}}\right|\right] \\
& \leqslant \mathrm{E}\left[\left|g^{\prime}\left(\bar{X}_{T}\right)\right|\left|\partial_{x} \bar{X}_{T}^{\bar{X}_{t_{n}}, t_{n}}-\partial_{x} X_{T}^{\bar{X}_{t_{n}}, t_{n}}\right|\right]+\mathrm{E}\left[\left|g^{\prime}\left(\bar{X}_{T}\right)-g^{\prime}\left(X_{T}^{\bar{X}_{t_{n}}, t_{n}}\right)\right|\left|\partial_{x} X_{T}^{\bar{X}_{t_{n}}, t_{n}}\right|\right] \\
& =\mathcal{O}\left(\check{N}^{-1 / 2}\right)
\end{aligned}
$$

A.2. Second, Third and Fourth Variations. The proof of Theorem 2.1 relies on bounded moments of higher order variations of the flow map $\varphi$. In this section, we we will verify that these higher order variations are indeed well defined random variables with all required moments bounded.

To this end, we define the following set of coupled SDE

$$
\begin{aligned}
d Y_{u}^{(1)}= & a\left(u, Y_{u}^{(1)}\right) d u+b\left(u, Y_{u}^{(1)}\right) d W_{u} \\
d Y_{u}^{(2)}= & a_{x}\left(u, Y_{u}^{(1)}\right) Y_{u}^{(2)} d u+b_{x}\left(u, Y_{u}^{(1)}\right) Y_{u}^{(2)} d W_{u} \\
d Y_{u}^{(3)}= & \left(a_{x x}\left(u, Y_{u}^{(1)}\right)\left(Y_{u}^{(2)}\right)^{2}+a_{x}\left(u, Y_{u}^{(1)}\right) Y_{u}^{(3)}\right) d u \\
& +\left(b_{x x}\left(u, Y_{u}^{(1)}\right)\left(Y_{u}^{(2)}\right)^{2}+b_{x}\left(u, Y_{u}^{(1)}\right) Y_{u}^{(3)}\right) d W_{u} \\
& \quad\left(a_{x x x}\left(u, Y_{u}^{(1)}\right)\left(Y_{u}^{(2)}\right)^{3}+3 a_{x x}\left(u, Y_{u}^{(1)}\right) Y_{u}^{(2)} Y_{u}^{(3)}+a_{x}\left(u, Y_{u}^{(1)}\right) Y_{u}^{(4)}\right) d u \\
& \quad+\left(b_{x x x}\left(u, Y_{u}^{(1)}\right)\left(Y_{u}^{(2)}\right)^{3}+3 b_{x x}\left(u, Y_{u}^{(1)}\right) Y_{u}^{(2)} Y_{u}^{(3)}+b_{x}\left(u, Y_{u}^{(1)}\right) Y_{u}^{(4)}\right) d W_{u} \\
& \quad+\left(a_{x x x x}\left(u, Y_{u}^{(1)}\right)\left(Y_{u}^{(2)}\right)^{4}+6 a_{x x x}\left(u, Y_{u}^{(1)}\right)\left(Y_{u}^{(2)}\right)^{2} Y_{u}^{(3)}\right) d u \\
d Y_{u}^{(5)}= & \quad\left(a_{x x}\left(u, Y_{u}^{(1)}\right)\left(3\left(Y_{u}^{(3)}\right)^{2}+4 Y_{u}^{(2)} Y_{u}^{(4)}\right)+a_{x}\left(u, Y_{u}^{(1)}\right) Y_{u}^{(5)}\right) d u \\
& \quad+\left(b_{x x}\left(u, Y_{u}^{(1)}\right)\left(3\left(Y_{u}^{(3)}\right)^{2}+4 Y_{u}^{(2)} Y_{u}^{(4)}\right)+b_{x}\left(u, Y_{u}^{(1)}\right) Y_{u}^{(5)}\right) d W_{u},
\end{aligned}
$$

defined for $u \in(t, T]$ with the initial condition $Y_{t}=(x, 1,0,0,0)$. The first component of the vector coincides with equation $(12)$, whereas the second one is the first variation of the path from equation (16). The last three components can be understood as the second, third and fourth variations of the path, respectively.

Making use of the solution of SDE (62), we also define the second, third and fourth variations as

$$
\begin{aligned}
\varphi_{x x}(t, x) & =g^{\prime}\left(X_{T}^{x, t}\right) \partial_{x x} X_{T}^{x, t}+g^{\prime \prime}\left(X_{T}^{x, t}\right)\left(\partial_{x} X_{T}^{x, t}\right)^{2}, \\
\varphi_{x x x}(t, x) & =g^{\prime}\left(X_{T}^{x, t}\right) \partial_{x x x} X_{T}^{x, t}+\cdots+g^{\prime \prime \prime}\left(X_{T}^{x, t}\right)\left(\partial_{x} X_{T}^{x, t}\right)^{3}, \\
\varphi_{x x x x}(t, x) & =g^{\prime}\left(X_{T}^{x, t}\right) \partial_{x x x x} X_{T}^{x, t}+\cdots+g^{\prime \prime \prime \prime}\left(X_{T}^{x, t}\right)\left(\partial_{x} X_{T}^{x, t}\right)^{4} .
\end{aligned}
$$

In the sequel, we prove that the solution to equation 62 when understood in the integral sense that extends $(12)$ is a well defined random variable with bounded moments. Given sufficient differentiability of the payoff $g$, this results in the boundedness of the higher order variations as required in Theorem 2.1 . 
Lemma A.1. Assume that (R.1), (R.2), and (R.3) in Theorem 2.1 hold and that for any fixed $t \in[0, T]$ and $x \in \mathcal{F}_{t}$ such that $\mathrm{E}\left[|x|^{2 p}\right]<\infty$ for all $p \in \mathbb{N}$. Then, equation 62 has pathwise unique solutions with finite moments. That is,

$$
\max _{i \in\{1,2, \ldots, 5\}}\left(\sup _{u \in[t, T]} \mathrm{E}\left[\left|Y_{u}^{(i)}\right|^{2 p}\right]\right)<\infty, \quad \forall p \in \mathbb{N} .
$$

Furthermore, the higher variations as defined by equation (63) satisfy

$$
\varphi_{x}(t, x), \varphi_{x x}(t, x), \varphi_{x x x}(t, x), \varphi_{x x x x}(t, x) \in \mathcal{F}_{T}
$$

and for all $p \in \mathbb{N}$,

$$
\max \left\{\mathrm{E}\left[\left|\varphi_{x}(t, x)\right|^{2 p}\right], \mathrm{E}\left[\left|\varphi_{x x}(t, x)\right|^{2 p}\right], \mathrm{E}\left[\left|\varphi_{x x x}(t, x)\right|^{2 p}\right], \mathrm{E}\left[\left|\varphi_{x x x x}(t, x)\right|^{2 p}\right]\right\}<\infty .
$$

Proof. We note that the system of SDE $(62)$ can be trivially truncated to its first $d_{1} \leqslant 5$ elements. That is, the truncated SDE for $\left\{Y_{u}^{(j)}\right\}_{j=1}^{d_{1}}$ for $d_{1}<5$ has drift and diffusion functions $\hat{a}:[0, T] \times$ $\mathbb{R}^{d_{1}} \rightarrow \mathbb{R}^{d_{1}}$ and $\hat{b}:[0, T] \times \mathbb{R}^{d_{1}} \rightarrow \mathbb{R}^{d_{1} \times d_{2}}$ that do not depend on $Y_{u}^{(j)}$ for $j \geqslant d_{1}$.

This enables verifying existence of solutions for the SDE in stages: first for $\left(Y^{(1)}, Y^{(2)}\right)$, thereafter for $\left(Y^{(1)}, Y^{(2)}, Y^{(3)}\right)$, and so forth, proceeding iteratively to add the next component $Y^{\left(d_{1}+1\right)}$ to the SDE. We shall also exploit this structure for proving the result of bounded moments for each component. The starting point for our proof is Lemma 2.2, which guarantees existence, uniqueness and the needed moment bounds for the first two components $Y^{(1)}$, and $Y^{(2)}$. It will turn out in the sequel that, thanks to the regularity in the drift and diffusion functions of the SDE (62), this regularity will cascade further to $Y^{(j)}$ for $j \in\{3,4,5\}$.

Kloeden and Platen [22, Theorems 4.5.3 and 4.5.4] note that their existence and uniqueness theorems for SDE cannot be modified in order to account for looser regularity conditions, and the proof below is a case in point. Our approach here follows closely the presentation of Kloeden and Platen, with slight modifications on the inequalities that are used to achieve bounds at various intermediate stages of the proof.

As a beginning stage for the proof, let us note that Theorem [20, Thorem 5.2.5] guarantees that the solutions of 62 are pahtwise unique and focus on verifying the claimed results for $Y_{u}^{(3)}$.

We define a successive set of approximations $Y_{u, n}^{(3)}, n \in \mathbb{N}$ by

$$
\begin{aligned}
Y_{u, n+1}^{(3)}=\int_{t}^{u} a_{x x}\left(s, Y_{s}^{(1)}\right)\left(Y_{s}^{(2)}\right)^{2}+a_{x}\left(s, Y_{s}^{(2)}\right) & Y_{s, n}^{(3)} d s \\
& +\int_{t}^{u} b_{x x}\left(s, Y_{s}^{(1)}\right)\left(Y_{s}^{(2)}\right)^{2}+b_{x}\left(s, Y_{s}^{(2)}\right) Y_{s, n}^{(3)} d W_{s},
\end{aligned}
$$

with the initial approximation defined by $Y_{u, 1}^{(3)}=0$, for all $u \in[t, T]$. Let us denote by

$$
Q=\int_{t}^{u} a_{x x}\left(s, Y_{s}^{(1)}\right)\left(Y_{s}^{(1)}\right)^{2} d s+\int_{t}^{u} b_{x x}\left(s, Y_{s}^{(1)}\right)\left(Y_{s}^{(2)}\right)^{2} d W_{s}
$$

the terms that do not depend on the, for the time being, highest order variation $Y_{u, n}^{(3)}$. We then have, using Young's inequality, that

$$
\begin{aligned}
\mathrm{E}\left[\left|Y_{u, n+1}^{(3)}\right|\right] & \leqslant 3 \mathrm{E}\left[|Q|^{2}\right]+3 \mathrm{E}\left[\left|\int_{t}^{u} a_{x}\left(s, Y_{s}^{(1)}\right) Y_{s, n}^{(3)} d s\right|^{2}\right]+3 \mathrm{E}\left[\left|\int_{t}^{u} b_{x}\left(s, Y_{s}^{(1)}\right) Y_{s, n}^{(3)} d W_{s}\right|^{2}\right] \\
& \leqslant 3 \mathrm{E}\left[|Q|^{2}\right]+3(u-t) \mathrm{E}\left[\int_{t}^{u}\left|a_{x}\left(s, Y_{s}^{(1)}\right) Y_{s, n}^{(3)}\right|^{2} d s\right]+3 \mathrm{E}\left[\int_{t}^{u}\left|b_{x}\left(s, Y_{s}^{(1)}\right) Y_{s, n}^{(3)}\right|^{2} d s\right] .
\end{aligned}
$$

The boundedness of the partial derivatives of the drift and diffusion terms in (12) gives us

$$
\mathrm{E}\left[\left|Y_{u, n+1}^{(3)}\right|^{2}\right] \leqslant 3 \mathrm{E}\left[|Q|^{2}\right]+C(u-t+1) \mathrm{E}\left[\int_{t}^{u}\left(1+\left|Y_{s, n}^{(3)}\right|^{2}\right) d s\right] .
$$


By induction, we consequently obtain that

$$
\sup _{t \leqslant u \leqslant T} \mathrm{E}\left[\left|Y_{u, n}^{(3)}\right|^{2}\right]<\infty, \quad \forall n \in \mathbb{N} .
$$

Now, we set $\Delta Y_{u, n}^{(3)}=Y_{u, n+1}^{(3)}-Y_{u, n}^{(3)}$. Then

$$
\begin{aligned}
\mathrm{E}\left[\left|\Delta Y_{u, n}^{(3)}\right|\right] & \leqslant 2 \mathrm{E}\left[\left|\int_{t}^{u} a_{x}\left(s, Y_{s}^{(1)}\right) \Delta Y_{s, n-1}^{(3)} d s\right|^{2}\right]+2 \mathrm{E}\left[\left|\int_{t}^{u} b_{x}\left(s, Y_{s}^{(1)}\right) \Delta Y_{s, n-1}^{(3)} d W_{s}\right|^{2}\right] \\
& \leqslant 2(u-t) \int_{t}^{u} \mathrm{E}\left[\left|a_{x}\left(s, Y_{s}^{(1)}\right) \Delta Y_{s, n-1}^{(3)}\right|^{2}\right] d s+2 \int_{t}^{u} \mathrm{E}\left[\left|b_{x}\left(s, Y_{s}^{(1)}\right) \Delta Y_{s, n-1}^{(3)}\right|^{2}\right] d s \\
& \leqslant C_{1} \int_{t}^{u} \mathrm{E}\left[\left|\Delta Y_{s, n-1}^{(3)}\right|^{2}\right] d s .
\end{aligned}
$$

Thus, by Grönwall's inequality,

$$
\mathrm{E}\left[\left|\Delta Y_{u, n}^{(3)}\right|^{2}\right] \leqslant \frac{C_{1}^{n-1}}{(n-1) !} \int_{t}^{u}(u-s)^{n-1} \mathrm{E}\left[\left|\Delta Y_{s, 1}^{(3)}\right|^{2}\right] d s .
$$

Let us next show that $\mathrm{E}\left[\left|\Delta Y_{s, 1}^{(3)}\right|^{2}\right]$ is bounded. First,

$$
\begin{aligned}
\mathrm{E}\left[\left|\Delta Y_{u, 1}^{(3)}\right|^{2}\right] & =\mathrm{E}\left[\left|\int_{t}^{u} a_{x}\left(s, Y_{s}^{(1)}\right) Y_{s, 2}^{(3)} d s+\int_{t}^{u} b_{x}\left(s, Y_{s}^{(1)}\right) Y_{u, 2}^{(3)} d W_{s}\right|^{2}\right] \\
& \leqslant C(u-t+1) \sup _{s \in[t, u]} \mathrm{E}\left[\left|Y_{s, 2}^{(3)}\right|^{2}\right] .
\end{aligned}
$$

Consequently, there exists a $C \in \mathbb{R}$ such that

$$
\mathrm{E}\left[\left|\Delta Y_{u, n}^{(3)}\right|^{2}\right] \leqslant \frac{C^{n}(u-t)^{n}}{n !}, \quad \sup _{u \in[t, T]} \mathrm{E}\left[\left|\Delta Y_{u, n}^{(3)}\right|^{2}\right] \leqslant \frac{C^{n}(T-t)^{n}}{n !}
$$

We define

$$
Z_{n}=\sup _{t \leqslant u \leqslant T}\left|\Delta Y_{u, n}^{(3)}\right|
$$

and note that

$$
\begin{aligned}
Z_{n} \leqslant & \int_{t}^{T}\left|a_{x}\left(s, Y_{s}^{(1)}\right) Y_{s, n+1}^{(3)}-a_{x}\left(s, Y_{s}^{(1)}\right) Y_{s, n}^{(3)}\right| d s \\
& +\sup _{t \leqslant u \leqslant T}\left|\int_{t}^{u} b_{x}\left(s, Y_{s}^{(1)}\right) Y_{s, n+1}^{(3)}-b_{x}\left(s, Y_{s}^{(1)}\right) Y_{s, n}^{(3)} d W_{s}\right|
\end{aligned}
$$

Using Doob's and Schwartz's inequalities, as well as the boundedness of $a_{x}$ and $b_{x}$,

$$
\begin{aligned}
\mathrm{E}\left[\left|Z_{n}\right|^{2}\right] \leqslant & 2(T-t) \int_{t}^{T} \mathrm{E}\left[\left|a_{x}\left(s, Y_{s}^{(1)}\right) Y_{s, n+1}^{(3)}-a_{x}\left(s, Y_{s}^{(1)}\right) Y_{s, n}^{(3)}\right|^{2}\right] d s \\
& +8 \int_{t}^{T} \mathrm{E}\left[\left|b_{x}\left(s, Y_{s}^{(1)}\right) Y_{s, n+1}^{(3)}-b_{x}\left(s, Y_{s}^{(1)}\right) Y_{s, n}^{(3)}\right|^{2}\right] d s \\
\leqslant & \frac{C^{n}(T-t)^{n}}{n !}
\end{aligned}
$$

for some $C \in \mathbb{R}$. Using the Markov inequality, we get

$$
\sum_{n=1}^{\infty} \mathrm{P}\left(Z_{n}>n^{-2}\right) \leqslant \sum_{n=1}^{\infty} \frac{n^{4} C^{n}(T-t)^{n}}{n !}
$$

The right-hand side of the equation above converges by the ratio test, whereas the Borel-Cantelli Lemma guarantees the (almost sure) existence of $K^{*} \in \mathbb{N}$, such that $Z_{k}<k^{2}, \forall k>K^{*}$. We 
conclude that $Y_{u, n}^{(3)}$ converges uniformly in $L^{2}(\mathrm{P})$ to the limit $Y_{u}^{(3)}=\sum_{n=1}^{\infty} \Delta Y_{u, n}^{(3)}$ and that since $\left\{Y_{u, n}^{(3)}\right\}_{n}$ is a sequence of continuous and $\mathcal{F}_{u^{-}}$adapted processes, $Y_{u}^{(3)}$ is also continuous and $\mathcal{F}_{u^{-}}$ adapted. Furthermore, as $n \rightarrow \infty$,

$$
\left|\int_{t}^{u} a_{x}\left(s, Y_{s}^{(1)}\right) Y_{s, n}^{(3)} d s-\int_{t}^{u} a_{x}\left(s, Y_{s}^{(1)}\right) Y_{s}^{(3)} d s\right| \leqslant C \int_{t}^{u}\left|Y_{s, n}^{(3)}-Y_{s}^{(3)}\right| d s \rightarrow 0, \quad \text { a.s. },
$$

and, similarly,

$$
\left|\int_{t}^{u} b_{x}\left(s, Y_{s}^{(1)}\right) Y_{s, n}^{(3)} d W_{s}-\int_{t}^{u} b_{x}\left(s, Y_{s}^{(1)}\right) Y_{s}^{(3)} d W_{s}\right| \rightarrow 0, \quad \text { a.s. }
$$

This implies that $Y_{u}^{(3)}$ is a solution to the SDE (62).

Having established that $Y_{u}^{(3)}$ solves the relevant SDE and that it has a finite second moment, we may follow the principles laid out in [22, Theorem 4.5.4] and show that all even moments of

$$
Y_{u}^{(3)}=Q+\int_{t}^{u} a_{x}\left(t, Y_{s}^{(1)}\right) Y_{s}^{(3)} d s+\int_{t}^{u} b_{x}\left(t, Y_{s}^{(1)}\right) Y_{s}^{(3)} d W_{s}
$$

are finite. By Itô's Lemma, we get that for any even integer $l$,

$$
\begin{aligned}
\left|Y_{u}^{(3)}\right|^{l}= & \int_{t}^{u}\left|Y_{s}^{(3)}\right|^{l-2} Y_{s}^{(3)}\left(a_{x x}\left(s, Y_{s}^{(1)}\right)\left(Y_{s}^{(2)}\right)^{2}+a_{x}\left(s, Y_{s}^{(1)}\right) Y_{s}^{(3)}\right) d s \\
& +\int_{t}^{u} \frac{l(l-1)}{2}\left|Y_{s}^{(3)}\right|^{l-2}\left(b_{x x}\left(s, Y_{s}^{(1)}\right)\left(Y_{s}^{(2)}\right)^{2}+b_{x}\left(s, Y_{s}^{(1)}\right) Y_{s}^{(3)}\right)^{2} d s \\
& +\int_{t}^{u}\left|Y_{s}^{(3)}\right|^{l-2} Y_{s}^{(3)}\left(b_{x x}\left(s, Y_{s}^{(1)}\right)\left(Y_{s}^{(2)}\right)^{2}+b_{x}\left(s, Y_{s}^{(1)}\right) Y_{s}^{(3)}\right) d W_{s} .
\end{aligned}
$$

Taking expectations, the Itô integral vanishes,

$$
\begin{aligned}
\mathrm{E}\left[\left|Y_{s}^{(3)}\right|^{l}\right]=\mathrm{E}\left[\int_{t}^{u}\left|Y_{s}^{(3)}\right|^{l-2} Y_{s}^{(3)}\left(a_{x x}\left(s, Y_{s}^{(1)}\right)\left(Y_{s}^{(2)}\right)^{2}+a_{x}\left(s, Y_{s}^{(1)}\right) Y_{s}^{(3)}\right) d s\right] \\
+\mathrm{E}\left[\int_{t}^{u} \frac{l(l-1)\left|Y_{s}^{(3)}\right|^{l-2}}{2}\left(b_{x x}\left(s, Y_{s}^{(1)}\right)\left(Y_{s}^{(2)}\right)^{2}+b_{x}\left(s, Y_{s}^{(1)}\right) Y_{s}^{(3)}\right)^{2} d s\right] .
\end{aligned}
$$

Using Young's inequality, denoting the term that does not depend on $Y_{s}^{(3)}$ in the first integral by $Q$, and exploiting the boundedness of $a_{x}$, we have that

$$
\begin{aligned}
\mathrm{E}\left[\left|Y_{u}^{(3)}\right|^{l}\right]-Q \leqslant & C \int_{t}^{u} \mathrm{E}\left[\left|Y_{3, u}\right|^{l}\right] d s \\
& +\mathrm{E}\left[\int_{t}^{u} \frac{l(l-1)\left|Y_{s}^{(3)}\right|^{l-2}}{2}\left(b_{x x}\left(s, Y_{s}^{(1)}\right)\left(Y_{s}^{(2)}\right)^{2}+b_{x}\left(s, Y_{s}^{(1)}\right) Y_{s}^{(3)}\right)^{2} d s\right] .
\end{aligned}
$$

By the same treatment for the latter integral, lumping together all terms independent of $Y_{s}^{(3)}$ and using that $b_{x}$ is bounded,

$$
\mathrm{E}\left[\left|Y_{u}^{(3)}\right|^{l}\right]-\tilde{Q} \leqslant C \int_{t}^{u} \mathrm{E}\left[\left|Y_{u}^{(3)}\right|^{l}\right] d s
$$

Thus, by Grönwall's inequality, $\mathrm{E}\left[\left|Y_{u}^{(3)}\right|^{l}\right]<\infty$.

Having established the existence and pathwise uniqueness of $Y_{s}^{(3)}$, as well as the finiteness of its moments, verifying the same properties for $Y_{u}^{(4)}$ and $Y_{u}^{(5)}$ can be done using similar arguments relying, most importantly, on the boundedness of the relevant derivatives of the drift and diffusion functions. Finally, the $\mathcal{F}_{T}$-measurability and moment bounds for the variations of the flow map $\varphi$ up to order four (cf. equation (63) ) can be verified by a straightforward extension of the argument in the proof of Lemma 2.2 . 
A.3. Error Expansion for the MSE in Multiple Dimensions. In this section, we extend the 1D MSE error expansion presented in Theorem 2.1 to the multi-dimensional setting.

Consider the SDE

$$
\begin{aligned}
d X_{t} & =a\left(t, X_{t}\right) d t+b\left(t, X_{t}\right) d W_{t}, \quad t \in(0, T] \\
X_{0} & =x_{0},
\end{aligned}
$$

where $X:[0, T] \rightarrow \mathbb{R}^{d_{1}}, W:[0, T] \rightarrow \mathbb{R}^{d_{2}}, a:[0, T] \times \mathbb{R}^{d_{1}} \rightarrow \mathbb{R}^{d_{1}}$ and $b:[0, T] \times \mathbb{R}^{d_{1}} \rightarrow \mathbb{R}^{d_{1} \times d_{2}}$. Let further $x_{i}$ denote the $i$-th component of $x \in \mathbb{R}^{d_{1}}, a^{(i)}$, the $i$-th component of a drift coefficient and $b^{(i, j)}$ and $b^{\mathrm{T}}$ denote the $(i, j)$-th element and the transpose of the diffusion matrix $b$, respectively. (To avoid confusion, this derivation does not make use of any MLMC notation, particularly not the multilevel superscript. $\{\ell\}$.)

Using the Einstein summation convention to sum over repeated indices, but not over the time index $n$, the $1 \mathrm{D}$ local error terms in equation (51) generalize into

$$
\begin{aligned}
& \widetilde{\Delta a_{n}^{(i)}}=\int_{t_{n}}^{t_{n+1}} \int_{t_{n}}^{t}\left(a_{t}^{(i)}+a_{x_{j}}^{(i)} a^{(j)}+\frac{1}{2} a_{x_{j} x_{k}}^{(i)}\left(b b^{T}\right)^{(j, k)}\right) d s d t, \\
& \widetilde{\Delta a_{n}^{(i)}}=\int_{t_{n}}^{t_{n+1}} \int_{t_{n}}^{t} a_{x_{j}}^{(i)} b^{(j, k)} d W_{s}^{(k)} d t, \\
& \widetilde{\Delta b}{ }_{n}^{(i)}=\int_{t_{n}}^{t_{n+1}} \int_{t_{n}}^{t} b_{t}^{(i, j)}+b_{x_{k}}^{(i, j)} a^{(k)}+\frac{1}{2} b_{x_{k} x_{\ell}}^{(i, j)}\left(b b^{T}\right)^{(k, \ell)} d s d W_{t}^{(j)}, \\
& \widetilde{\Delta b}{ }_{n}^{(i)}=\int_{t_{n}}^{t_{n+1}} \int_{t_{n}}^{t} b_{x_{k}}^{(i, j)} b^{(k, \ell)} d W_{s}^{(\ell)} d W_{t}^{(j)},
\end{aligned}
$$

where all the above integrand functions in all equations implicitly depend on the state argument $X_{s}^{\bar{X}_{t_{n}}, t_{n}}$. In flow notation, $a_{t}^{(i)}$ is shorthand for $a_{t}^{(i)}\left(s, X_{s}^{\bar{X}_{t_{n}}, t_{n}}\right)$.

Under sufficient regularity, a tedious calculation similar to the proof of Theorem 2.1 verifies that, for a given smooth payoff, $g: \mathbb{R}^{d_{1}} \rightarrow \mathbb{R}$,

$$
\mathrm{E}\left[\left(g\left(X_{T}\right)-g\left(\bar{X}_{T}\right)\right)^{2}\right] \leqslant \mathrm{E}\left[\sum_{n=0}^{N-1} \bar{\rho}_{n} \Delta t_{n}^{2}+o\left(\Delta t_{n}^{2}\right)\right],
$$

where

$$
\bar{\rho}_{n}:=\frac{1}{2} \bar{\varphi}_{x_{i}, n}\left(\left(b b^{\mathrm{T}}\right)^{(k, \ell)}\left(b_{x_{k}} b_{x_{\ell}}^{\mathrm{T}}\right)\right)^{(i, j)}\left(t_{n}, \bar{X}_{t_{n}}\right) \bar{\varphi}_{x_{j}, n} .
$$

In the multi-dimensional setting, the $i$-th component of first variation of the flow map, $\varphi_{x}=$ $\left(\varphi_{x_{1}}, \varphi_{x_{2}}, \ldots, \varphi_{x_{d_{1}}}\right)$, is given by

$$
\varphi_{x_{i}}(t, y)=g_{x_{j}}\left(X_{T}^{y, t}\right) \partial_{x_{i}}\left(X_{T}^{y, t}\right)^{(j)} .
$$

The first variation is defined as the second component to the solution of the SDE,

$$
\begin{aligned}
d Y_{s}^{(1, i)} & =a^{(i)}\left(s, Y_{s}^{(1)}\right) d s+b^{(i, j)}\left(s, Y_{s}^{(1)}\right) d W_{s}^{(j)} \\
d Y_{s}^{(2, i, j)} & =a_{x_{k}}^{(i)}\left(s, Y_{s}^{(1)}\right) Y_{s}^{(2, k, j)} d s+b_{x_{k}}^{(i, \ell)}\left(s, Y_{s}^{(1)}\right) Y_{s}^{(2, k, j)} d W_{s}^{(\ell)},
\end{aligned}
$$

where $s \in(t, T]$ and the initial conditions are given by $Y_{t}^{(1)}=x \in \mathbb{R}^{d_{1}}, Y_{t}^{(2)}=I_{d_{1}}$, with $I_{d_{1}}$ denoting the $d_{1} \times d_{1}$ identity matrix. Moreover, the extension of the numerical method for solving the first variation of the 1D flow map (25) reads

$$
\begin{aligned}
\bar{\varphi}_{x_{i}, n} & =c_{x_{i}}^{(j)}\left(t_{n}, \bar{X}_{t_{n}}\right) \bar{\varphi}_{x_{j}, n+1}, \quad n=N-1, N-2, \ldots 0 . \\
\bar{\varphi}_{x_{i}, N} & =g_{x_{i}}\left(\bar{X}_{T}\right),
\end{aligned}
$$

with the $j$-th component of $c:[0, T] \times \mathbb{R}^{d_{1}} \rightarrow \mathbb{R}^{d_{1}}$ defined by

$$
c^{(j)}\left(t_{n}, \bar{X}_{t_{n}}\right)=\bar{X}_{t_{n}}^{(j)}+a^{(j)}\left(t_{n}, \bar{X}_{t_{n}}\right) \Delta t_{n}+b^{(j, k)}\left(t_{n}, \bar{X}_{t_{n}}\right) \Delta W_{n}^{(k)} .
$$


Let $U$ and $V$ denote subsets of Euclidean spaces and let us introduce the multi-index $\nu=$ $\left(\nu_{1}, \nu_{2}, \ldots, \nu_{d}\right)$ to represent spatial partial derivatives of order $|\nu|:=\sum_{j=1}^{d} \nu_{j}$ on the following short form $\partial_{x_{\nu}}:=\prod_{j=1}^{d} \partial_{x_{j}}^{\nu}$. We further introduce the following function spaces.

$$
\begin{aligned}
& C(U ; V):=\{f: U \rightarrow V \mid f \text { is continuous }\}, \\
& C_{b}(U ; V):=\{f: U \rightarrow V \mid f \text { is continuous and bounded }\}, \\
& C_{b}^{k}(U ; V):=\left\{f: U \rightarrow V \mid f \in C(U ; V) \text { and } \frac{d^{j}}{d x^{j}} f \in C_{b}(U ; V)\right. \\
& \quad \text { for all integers } 1 \leqslant j \leqslant k\}, \\
& C_{b}^{k_{1}, k_{2}}([0, T] \times U ; V):=\{f:[0, T] \times U \rightarrow V \mid f \in C([0, T] \times U ; V), \text { and } \\
& \left.\quad \partial_{t}^{j} \partial_{\nu} f \in C_{b}([0, T] \times U ; V) \text { for all integers s.t. } j \leqslant k_{1} \text { and } 1 \leqslant j+|\nu| \leqslant k_{2}\right\} .
\end{aligned}
$$

Theorem A.2 (MSE leading order error expansion in the multi-dimensional setting). Assume that drift and diffusion coefficients and input data of the SDE 65 fulfill

(R.1) $a \in C_{b}^{2,4}\left([0, T] \times \mathbb{R}^{d_{1}} ; \mathbb{R}^{d_{1}}\right)$ and $b \in C_{b}^{2,4}\left([0, T] \times \mathbb{R}^{d_{1}} ; \mathbb{R}^{d_{1} \times d_{2}}\right)$,

(R.2) there exists a constant $C>0$ such that

$$
|a(t, x)|^{2}+|b(t, x)|^{2} \leqslant C\left(1+|x|^{2}\right), \quad \forall x \in \mathbb{R}^{d_{1}} \text { and } \forall t \in[0, T],
$$

(R.3) $g^{\prime} \in C_{b}^{3}\left(\mathbb{R}^{d_{1}}\right)$ and there exists a $k \in \mathbb{N}$ such

$$
|g(x)|+\left|g^{\prime}(x)\right| \leqslant C\left(1+|x|^{k}\right), \quad \forall x \in \mathbb{R}^{d_{1}},
$$

(R.4) for the initial data, $X_{0} \in \mathcal{F}_{0}$ and $\mathrm{E}\left[\left|X_{0}\right|^{p}\right]<\infty$ for all $p \geqslant 1$.

Assume further the mesh points $0=t_{0}<t_{1}<\ldots<t_{N}=T$

(M.1) are stopping times for which $t_{n} \in \mathcal{F}_{t_{n-1}}$ for $n=1,2, \ldots, N$,

(M.2) for all mesh realizations, there exists a deterministic integer, $\check{N}$, and a $c_{1}>0$ such that $c_{1} \check{N} \leqslant N \leqslant \check{N}$ and a $c_{2}>0$ such that $\max _{n \in\{0,1, \ldots, N-1\}} \Delta t_{n}<c_{2} \check{N}^{-1}$,

(M.3) and there exists a $c_{3}>0$ such that for all $p \in[1,8]$ and $n \in\{0,1, \ldots, \Sigma=1\}$,

$$
\mathrm{E}\left[\Delta t_{n}^{2 p}\right] \leqslant c_{3}\left(\mathrm{E}\left[\Delta t_{n}^{2}\right]\right)^{p} .
$$

Then, as $\check{N}$ increases,

$$
\mathrm{E}\left[\left(g\left(X_{T}\right)-g\left(\bar{X}_{T}\right)\right)^{2}\right]=\mathrm{E}\left[\sum_{n=0}^{N-1} \frac{\left(\varphi_{x_{i}}\left(\left(b b^{\mathrm{T}}\right)^{(k, \ell)}\left(b_{x_{k}} b_{x_{\ell}}^{\mathrm{T}}\right)\right)^{(i, j)} \varphi_{x_{j}}\right)\left(t_{n}, \bar{X}_{t_{n}}\right)}{2} \Delta t_{n}^{2}+o\left(\Delta t_{n}^{2}\right)\right],
$$

where we have dropped the arguments of the first variation as well as the diffusion matrices for clarity.

Replacing the first variation $\varphi_{x_{i}}\left(t_{n}, \bar{X}_{n}\right)$ by the numerical approximation $\bar{\varphi}_{x_{i}, n}$, as defined in (67) and using the error density notation $\bar{\rho}$ from (66), we obtain the following to leading order all-terms-computable error expansion:

$$
\mathrm{E}\left[\left(g\left(X_{T}\right)-g\left(\bar{X}_{T}\right)\right)^{2}\right]=\mathrm{E}\left[\sum_{n=0}^{N-1} \bar{\rho}_{n} \Delta t_{n}^{2}+o\left(\Delta t_{n}^{2}\right)\right] .
$$

\section{Appendix B. A Uniform Time Step MLMC Algorithm}

The uniform time step MLMC algorithm for MSE approximations of SDE was proposed in 8 . Below, we present the version of that method that we use in the numerical tests in this work for reaching the approximation goal (2).

\section{Acknowledgments.}

The authors would like to thank Arturo Kohatsu-Higa for his helpful suggestions for improvements in the proof of Theorem 2.1 . 


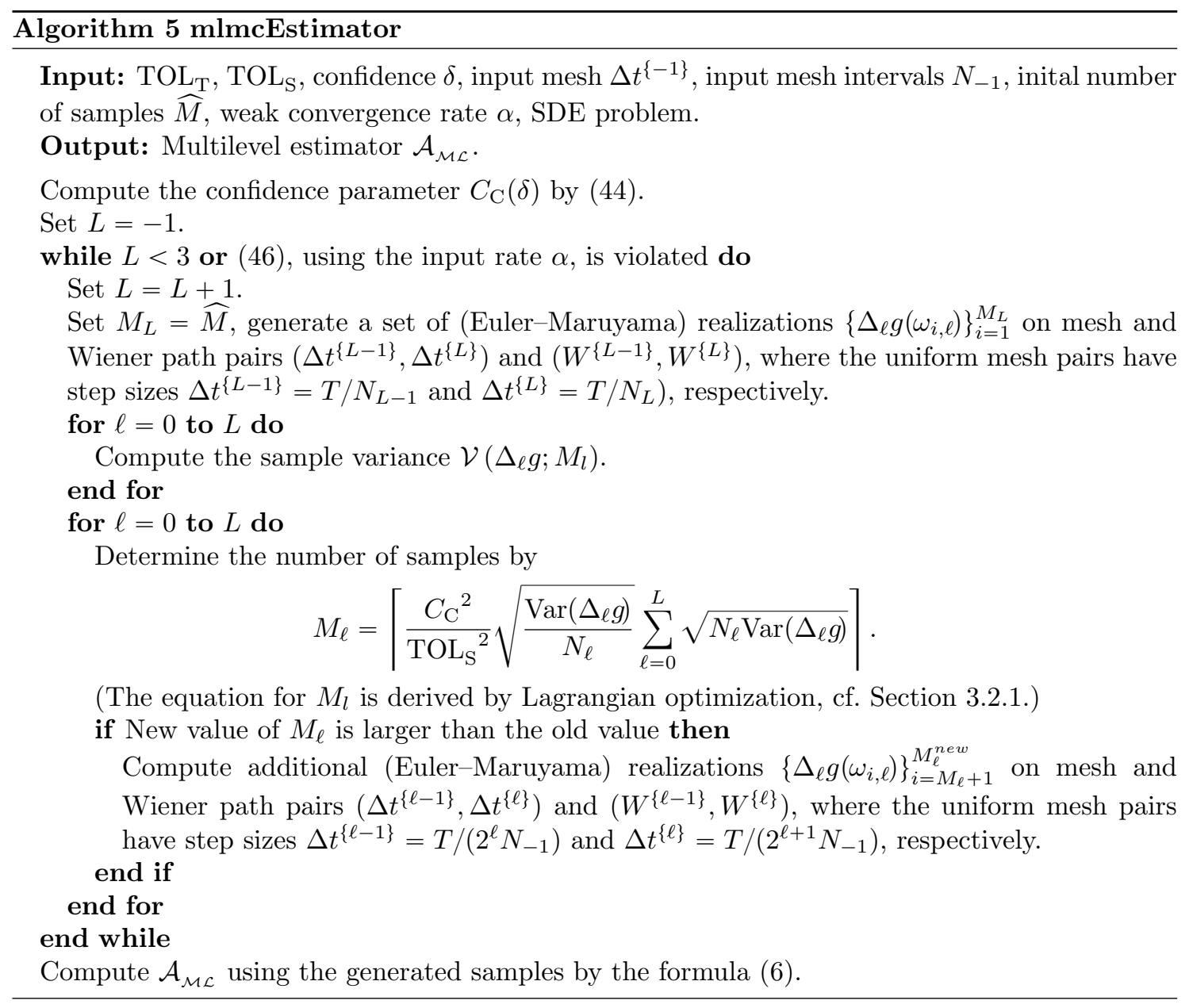

Support This work was supported by King Abdullah University of Science and Technology (KAUST); by Norges Forskningsråd, research project 214495 LIQCRY; and by the University of Texas, Austin Subcontract (Project Number 024550, Center for Predictive Computational Science). The first author was and the third author is a member of the Strategic Research Initiative on Uncertainty Quantification in Computational Science and Engineering at KAUST (SRI-UQ).

\section{REFERENCES}

[1] R. Avikainen. On irregular functionals of SDEs and the Euler scheme. Finance and Stochastics, 13(3):381-401, 2009.

[2] W. Bangerth and R. Rannacher. Adaptive finite element methods for differential equations. Lectures in Mathematics ETH Zürich. Birkhäuser Verlag, Basel, 2003.

[3] A. Barth and A. Lang. Multilevel Monte Carlo method with applications to stochastic partial differential equations. Int. J. Comput. Math., 89(18):2479-2498, 2012.

[4] K. A. Cliffe, M. B. Giles, R. Scheichl, and A. L. Teckentrup. Multilevel Monte Carlo methods and applications to elliptic PDEs with random coefficients. Comput. Vis. Sci., 14(1):3-15, 2011.

[5] Nathan Collier, Abdul-Lateef Haji-Ali, Fabio Nobile, Erik von Schwerin, and Raúl Tempone. A continuation multilevel monte carlo algorithm. BIT Numerical Mathematics, 55(2):399-432, 2014.

[6] R. Durrett. Probability: theory and examples. Duxbury Press, Belmont, CA, second edition, 1996.

[7] J. G. Gaines and T. J. Lyons. Variable step size control in the numerical solution of stochastic differential equations. SIAM J. Appl. Math, 57:1455-1484, 1997.

[8] M. B. Giles. Multilevel Monte Carlo path simulation. Oper. Res., 56(3):607-617, 2008.

[9] M. B. Giles and L. Szpruch. Antithetic multilevel Monte Carlo estimation for multi-dimensional SDEs without Lévy area simulation. Ann. Appl. Probab., 24(4):1585-1620, 2014.

[10] Michael B Giles. Multilevel monte carlo methods. Acta Numerica, 24:259-328, 2015.

[11] D. T. Gillespie. The chemical Langevin equation. The Journal of Chemical Physics, 113(1):297-306, 2000. 
[12] P. Glasserman. Monte Carlo methods in financial engineering, volume 53 of Applications of Mathematics (New York). Springer-Verlag, New York, 2004. Stochastic Modelling and Applied Probability.

[13] Abdul-Lateef Haji-Ali, Fabio Nobile, Erik von Schwerin, and Ral Tempone. Optimization of mesh hierarchies in multilevel monte carlo samplers. Stochastic Partial Differential Equations: Analysis and Computations, pages 1-37, 2015.

[14] S. Heinrich. Monte Carlo complexity of global solution of integral equations. J. Complexity, 14(2):151-175, 1998.

[15] S. Heinrich and E. Sindambiwe. Monte Carlo complexity of parametric integration. J. Complexity, 15(3):317341, 1999.

[16] H. Hoel, E. von Schwerin, A. Szepessy, and R. Tempone. Implementation and analysis of an adaptive multilevel Monte Carlo algorithm. Monte Carlo Methods and Applications, 20(1):1-41, 2014.

[17] N. Hofmann, T. Müller-Gronbach, and K. Ritter. Optimal approximation of stochastic differential equations by adaptive step-size control. Math. Comp., 69(231):1017-1034, 2000.

[18] J. D. Hunter. Matplotlib: A 2d graphics environment. Computing in Science Engineering, 9(3):90 -95, mayjune 2007.

[19] S. Ilie. Variable time-stepping in the pathwise numerical solution of the chemical Langevin equation. The Journal of Chemical Physics, 137(23):-, 2012.

[20] I. Karatzas and S. E. Shreve. Brownian motion and stochastic calculus, volume 113 of Graduate Texts in Mathematics. Springer-Verlag, New York, second edition, 1991.

[21] A. Kebaier. Statistical Romberg extrapolation: A new variance reduction method and applications to option pricing. Ann. Appl. Probab., 15(4):2681-2705, 2005.

[22] P. E. Kloeden and E. Platen. Numerical solution of stochastic differential equations, volume 23 of Applications of Mathematics (New York). Springer-Verlag, Berlin, 1992.

[23] H. Lamba, J. C. Mattingly, and A. M. Stuart. An adaptive Euler-Maruyama scheme for SDEs: convergence and stability. IMA J. Numer. Anal., 27(3):479-506, 2007.

[24] P. L'Ecuyer and E. Buist. Simulation in Java with SSJ. In Proceedings of the 37th conference on Winter simulation, WSC '05, pages 611-620. Winter Simulation Conference, 2005.

[25] G. N. Milstein and M. V. Tretyakov. Quasi-symplectic methods for Langevin-type equations. IMA J. Numer. Anal., 23(4):593-626, 2003.

[26] S. Mishra and C. Schwab. Sparse tensor multi-level Monte Carlo finite volume methods for hyperbolic conservation laws with random initial data. Math. Comp., 81(280):1979-2018, 2012.

[27] B. Øksendal. Stochastic differential equations. Universitext. Springer-Verlag, Berlin, fifth edition, 1998.

[28] E. Platen and D. Heath. A benchmark approach to quantitative finance. Springer Finance. Springer-Verlag, Berlin, 2006.

[29] S. E. Shreve. Stochastic calculus for finance. II. Springer Finance. Springer-Verlag, New York, 2004. Continuous-time models.

[30] R. D. Skeel and J. A. Izaguirre. An impulse integrator for Langevin dynamics. Molecular Physics, 100(24):38853891, 2002.

[31] A. Szepessy, R. Tempone, and G. E. Zouraris. Adaptive weak approximation of stochastic differential equations. Comm. Pure Appl. Math., 54(10):1169-1214, 2001.

[32] D. Talay. Stochastic Hamiltonian systems: exponential convergence to the invariant measure, and discretization by the implicit Euler scheme. Markov Process. Related Fields, 8(2):163-198, 2002. Inhomogeneous random systems (Cergy-Pontoise, 2001).

[33] D. Talay and L. Tubaro. Expansion of the global error for numerical schemes solving stochastic differential equations. Stochastic Analysis and Applications, 8(4):483-509, 1990.

[34] A. L. Teckentrup, R. Scheichl, M. B. Giles, and E. Ullmann. Further analysis of multilevel Monte Carlo methods for elliptic PDEs with random coefficients. Numer. Math., 125(3):569-600, 2013.

[35] L. Yan. The Euler scheme with irregular coefficients. The Annals of Probability, 30(3):1172-1194, 2002. 